2017

\title{
Experimental Evaluation of Two OpenFlow Controllers
}

\author{
Darianian, Mohamad
}

Darianian, M. (2017). Experimental Evaluation of Two OpenFlow Controllers (Unpublished master's thesis). University of Calgary, Calgary, AB. doi:10.11575/PRISM/26059

http://hdl.handle.net/11023/3911

Downloaded from PRISM Repository, University of Calgary 


\title{
UNIVERSITY OF CALGARY
}

\author{
Experimental Evaluation of Two OpenFlow Controllers
}

by

Mohamad Darianian

\begin{abstract}
A THESIS
SUBMITTED TO THE FACULTY OF GRADUATE STUDIES

IN PARTIAL FULFILLMENT OF THE REQUIREMENTS FOR THE

DEGREE OF MASTER OF SCIENCE
\end{abstract}

GRADUATE PROGRAM IN COMPUTER SCIENCE

CALGARY, ALBERTA

June, 2017

(C) Mohamad Darianian 2017 


\begin{abstract}
Network management has become a tricky task in today's complex networks and distributed data centers. Software Defined Networking (SDN) provides more flexibility, eases automation, and gives a more comprehensive view of the network. In a software-defined network, traffic management functionality requires a high-performance and responsive controller.

The SDN controller, as the "brain" of the network, enables network administrators to classify, manipulate, and dynamically re-route an evolving set of traffic flows across many possible network paths. In mission-critical networks, having a flexible and carrier-grade controller is a high priority.

In this thesis, we conduct an experimental evaluation of two open-source distributed OpenFlow controllers, namely ONOS and OpenDaylight. To this end, we construct a testbed and use a standard benchmarking tool called Cbench to evaluate their performance. We benchmark the throughput and latency of these controllers in both physical and virtual environments. The experimental results show that ONOS provides higher throughput and lower latency than OpenDaylight.
\end{abstract}




\section{Acknowledgements}

I wish to express my sincere gratitude toward my supervisor, Carey Williamson. I recall the day when I cold emailed Carey indicating my desires in this area, and was thrilled with his prompt and positive response. From the very first meeting, he has always been incredibly kind, professional, and extremely flexible. His guidance, magic touch, and invaluable feedback on my drafts significantly improved the quality of this thesis. Thanks Carey for the opportunity of a lifetime.

I heartily thank Ali Tizghadam. He has been a mentor of unparalleled value to me. I cherish his advice and guidance. He helped me a lot in shaping the idea of this study, and was quite friendly and supportive throughout my time at TELUS. He deserves a high credit for enabling this thesis.

Along the journey, I had the privilege to interact with some skilled individuals that helped me in various ways. I would like to thank Hadi Bannazadeh for technical contribution in building the testbed, and Saeed Pouryazdian that helped me in some math portion of data post-processing. I specifically would like to thank Thomas Vachuska, You Wang, Luis Gomez, and Ola Salman for their responsiveness to my technical inquiries.

Ahmad Pakdaman deserves a special mention as a good friend of mine for sharing his unique and useful graduate studies experience, and willingness to discuss my problems and concerns. Also, I wish to thank my good fellows in Calgary. Ali Mohebbian, for his great hospitality, and Mohammad Pakdaman, who facilitated getting the revised drafts from my supervisor since I spent most of my program in Toronto.

Last but not least, I wish to express my heartfelt appreciation toward my parents and my sister. We were physically distant but emotionally close. I always benefited from their unconditional love, unflagging support, and unflinching encouragement throughout my life and the entire program. 


\section{Table of Contents}

Abstract ....................................... ii

Acknowledgements . . . . . . . . . . . . . . . . iii

Table of Contents . . . . . . . . . . . . . . . . . . . . . . . . iv

List of Tables . . . . . . . . . . . . . . . . . . . . . . . . . vi

List of Figures . . . . . . . . . . . . . . . . . . . . . . v vii

List of Symbols . . . . . . . . . . . . . . . . . . . . . . . . ix

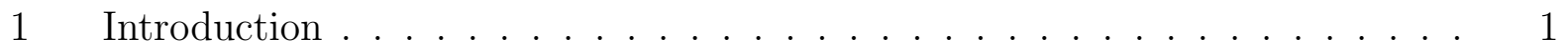

1.1 Background . . . . . . . . . . . . . . . . . . . . . . . . . 1

1.2 Motivation . . . . . . . . . . . . . . . . . . . . . . . 3

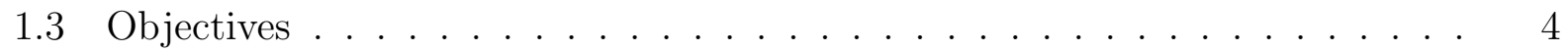

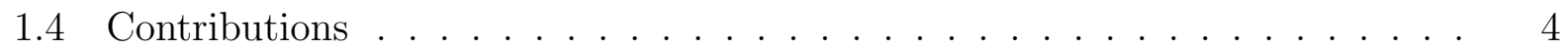

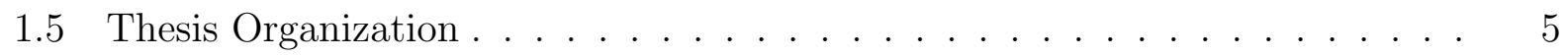

2 Background and Related Work . . . . . . . . . . . . . . . 6

2.1 Overview . . . . . . . . . . . . . . . . . . . . . . . 6

2.2 Software-Defined Networking (SDN) $\ldots \ldots \ldots \ldots \ldots$

2.2 .1 SDN Architecture . . . . . . . . . . . . . . . . . . . . . . . . 8

2.2 .2 Infrastructure Layer $\ldots \ldots \ldots$

2.2 .3 Southbound API $\ldots \ldots \ldots \ldots \ldots$

2.2 .4 Control Layer . . . . . . . . . . . . . . . . . . . . . . 11

2.2 .5 Northbound API . . . . . . . . . . . . . . . . . . . . . . . . . . . . 12

$2.2 .6 \quad$ Applications and Services $\ldots \ldots \ldots$

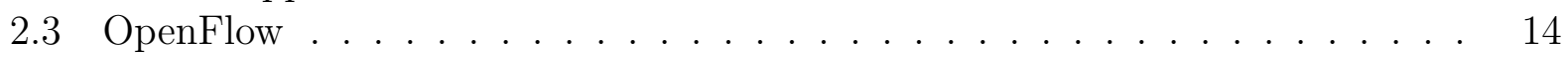

2.3 .1 OpenFlow Messages $\ldots \ldots \ldots \ldots$

$2.3 .2 \quad$ OpenFlow Controller Modes of Operation . . . . . . . . . . . . 21

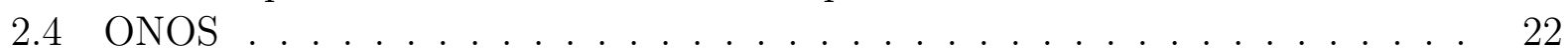

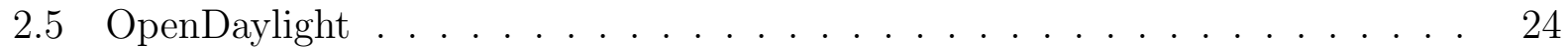

2.6 Virtualization . . . . . . . . . . . . . . . . . . . . . . . . . . . . . . . . . . 26

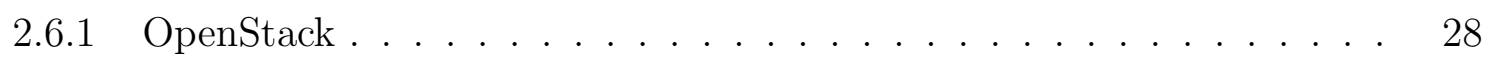

2.7 Hyper-Threading . . . . . . . . . . . . . . . . . . . . . . . . . . . . . . . . . . 31

2.8 Related Work in Performance of OpenFlow Controllers . . . . . . . . . . . . 33

2.9 Summary $\ldots \ldots \ldots \ldots \ldots \ldots \ldots$

$3 \quad$ Methodology and Experiment Setup. . . . . . . . . . . . . . . . . . 38

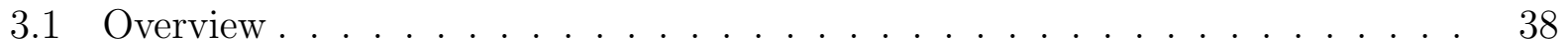

3.2 Methodology and Metrics $\ldots \ldots \ldots \ldots$

3.2 .1 Throughput, Latency, and Thread Scalability $\ldots \ldots \ldots$

3.3 Testbed Setup . . . . . . . . . . . . . . . . . . . . . . . . . . . . 40

3.4 Experimental Setup . . . . . . . . . . . . . . . . . . . . . . . . . . . . 46

$3.4 .1 \quad$ Experimental Factors . . . . . . . . . . . . . . . . . . . . . . . . . 47

3.4 .2 Structure of Experiments . . . . . . . . . . . . . . . . . . . . . 47

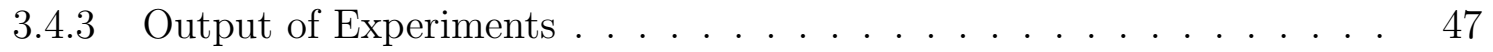

3.5 Cbench . . . . . . . . . . . . . . . . . . . . . . . . . . 49

3.6 Summary $\ldots \ldots \ldots \ldots$ 
$4 \quad$ Baseline Experimental Results . . . . . . . . . . . . . . . . . . 50

4.1 Overview . . . . . . . . . . . . . . . . . . . . . . 50

4.2 Throughput Results . . . . . . . . . . . . . . . . . . . . . . . . . 52

4.3 Latency Results . . . . . . . . . . . . . . . . . . . . . . . . . . . . . 64

4.4 Thread Scalability Results . . . . . . . . . . . . . . . . . . . . . 73

4.5 Summary . . . . . . . . . . . . . . . . . . . . . . . . . 80

5 $\quad$ Additional Experimental Results . . . . . . . . . . . . . . . . . . . . 81

5.1 Memory Usage Results . . . . . . . . . . . . . . . . . . . . . . . . . . . . 81

$5.2 \quad$ CPU Usage Results . . . . . . . . . . . . . . . . . . . . . . . . . . . . . . . . 94

5.3 Thread Scalability Results with Threads on One Socket . . . . . . . . . . . . 103

5.4 Summary . . . . . . . . . . . . . . . . . . . . . . 105

6 Conclusions . . . . . . . . . . . . . . . . . . . . . . . . 107

6.1 Thesis Summary . . . . . . . . . . . . . . . . . . . . 107

6.2 Conclusions . . . . . . . . . . . . . . . . . . . . . . . . 108

6.3 Future Work . . . . . . . . . . . . . . . . . . . . . . . . . . . 109

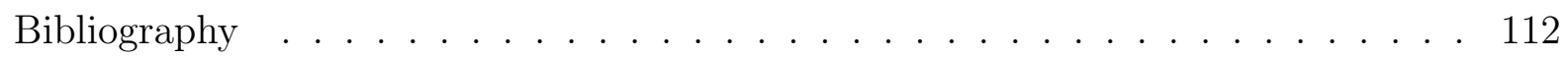

A Controllers Settings for Performance Testing . . . . . . . . . . . . . . . . . . 123

A.1 ONOS . . . . . . . . . . . . . . . . . . . . . . . 123

A.2 OpenDaylight . . . . . . . . . . . . . . . . . . . . 124

A.3 Cbench Commands . . . . . . . . . . . . . . . . . . . . . . . . . . 125

A.4 Overall Mean and Standard Deviation Calculation . . . . . . . . . . . . . . . 126

A.5 Copyright Permission . . . . . . . . . . . . . . . . . . . 127 


\section{List of Tables}

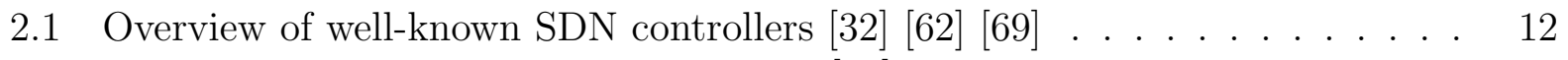

2.2 OpenFlow major changes in each release $42 \pi \ldots \ldots$. . . . . . . . . . . . 18

3.1 Total CPU cores and threads per server (HT enabled) . . . . . . . . . . . . . 40

3.2 Total CPU cores and threads per server (HT disabled) . . . . . . . . . . . . 40

3.3 Controller and Cbench VM specifications . . . . . . . . . . . . . . . . . . . . 42

3.4 Rounds of tests per experiment . . . . . . . . . . . . . . . . . . 47

4.1 Parameters for throughput tests . . . . . . . . . . . . . . . . . . . 51

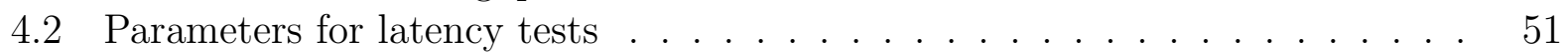

4.3 Parameters for thread scalability tests . . . . . . . . . . . . . . . . 51

4.4 ONOS throughput comparison with prior work [80] . . . . . . . . . . . . 55

4.5 OpenDaylight throughput failed tests . . . . . . . . . . . . . . . 58

$4.6 \quad$ OpenDaylight throughput comparison with prior work $[60]$. . . . . . . . . 58

$4.7 \quad$ ONOS and OpenDaylight throughput comparison with [83] . . . . . . . . . . 61

$4.8 \quad$ ONOS and OpenDaylight latency comparison with prior work [80] . . . . . . 68

4.9 Comparing OpenDaylight latency failed tests ratio with prior work $\mid 60]$. . . 68

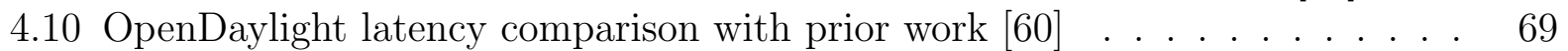

4.11 ONOS and OpenDaylight flow setup latency comparison with [99] . . . . . . 69

4.12 ONOS and OpenDaylight flow setup latency comparison with [87] . . . . . 70

4.13 ONOS throughput comparison with prior work $[99] \mid$. . . . . . . . . . . . . 77

4.14 OpenDaylight throughput comparison with prior work $[99]$. . . . . . . . . . 77

4.15 ONOS latency comparison with prior work [99]] . . . . . . . . . . . . . . 80

4.16 OpenDaylight latency comparison with prior work $[99]$. . . . . . . . . . . . 80

A.1 Cbench Command Options Description . . . . . . . . . . . . . . . 125 


\section{List of Figures and Illustrations}

1.1 The role of a controller in software-defined networks [69] . . . . . . . . . . 2

2.1 Traditional network architecture versus Software-Defined Network $|69|$. . . . 7

$2.2 \quad$ SDN high-level architecture $[57] \ldots \ldots \ldots$

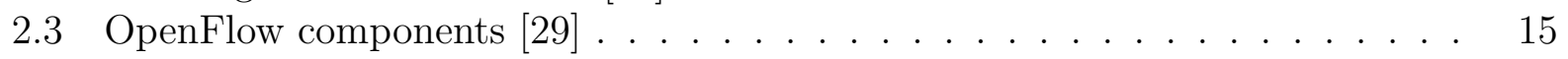

2.4 Schematic view of OpenFlow device and OpenFlow table 62$] \ldots$. . . . . . . 16

2.5 Packet flow structure in the OpenFlow switch $[29]$. . . . . . . . . . . . . 17

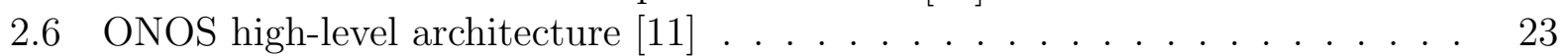

2.7 OpenDaylight high-level architecture [14] . . . . . . . . . . . . . . . . . 25

2.8 Traditional and virtual architecture . . . . . . . . . . . . . . . . . . . . . . . 26

2.9 Conceptual view of OpenStack's main components [18] . . . . . . . . . . . . 30

2.10 Hyper-Threading technology concept $[5]$. . . . . . . . . . . . . . . . 31

3.1 Physical testbed structure . . . . . . . . . . . . . . . . . . . . . 41

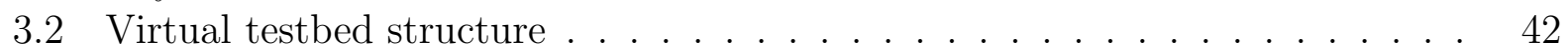

$3.3 \quad$ Physical/Virtual network of the controller and Cbench VMs . . . . . . . . . 43

3.4 TCP throughput test between Cbench and controller VM . . . . . . . . . . . 45

3.5 Hyper-Threading option in the BIOS menu . . . . . . . . . . . . . . . . . . 46

$4.1 \quad$ ONOS average throughput with different number of switches . . . . . . . . 53

$4.2 \quad$ OpenDaylight average throughput with different number of switches . . . . . 53

$4.3 \quad$ Example of OpenDaylight throughput tests with 256 and 512 switches . . . . 57

4.4 OpenDaylight average throughput with different number of switches using 16 and 24 threads . . . . . . . . . . . . . . . . . . . . . . . 60

4.5 ONOS average throughput with different number of hosts . . . . . . . . . . . 63

$4.6 \quad$ OpenDaylight average throughput with different number of hosts . . . . . . . 63

4.7 ONOS average flow setup latency with different number of switches . . . . . 65

4.8 OpenDaylight average flow setup latency with different number of switches . 65

4.9 ONOS average flow setup latency with different number of hosts . . . . . . . 72

4.10 OpenDaylight average flow setup latency with different number of hosts . . . 72

4.11 ONOS average throughput with different number of threads $\ldots . . . .75$

4.12 OpenDaylight average throughput with different number of threads . . . . . 75

4.13 ONOS average flow setup latency with different number of threads . . . . . . 78

4.14 OpenDaylight average flow setup latency with different number of threads . $\quad 78$

5.1 Garbage collection generations . . . . . . . . . . . . . . . . . . 82

5.2 Schematic view of Heap memory before and after minor GC . . . . . . . . . 84

$5.3 \quad$ Schematic view of Heap memory before and after full GC . . . . . . . . . . . 84

$5.4 \quad$ YourKit profiler's prompt when successfully attached to the controller . . . . 85

$5.5 \quad$ OpenDaylight Heap memory usage under throughput test . . . . . . . . . . . 87

$5.6 \quad$ OpenDaylight GC generations in throughput test . . . . . . . . . . . . . . . 89

5.7 ONOS's Heap memory usage under throughput test . . . . . . . . . . . . . . 91

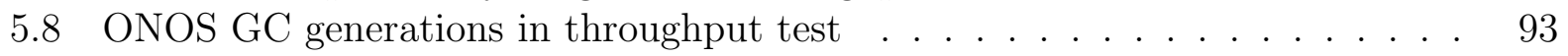


5.9 ONOS and OpenDaylight CPU usage under throughput test . . . . . . . . 101

5.10 ONOS average TP with different number of threads (1 socket vs 2 sockets) 104

5.11 ODL average TP with different number of threads (1 socket vs 2 sockets)] . . 104

A.1 Copyright permission of MATLAB function and code . . . . . . . . . . . 127 


\section{List of Acronyms}

Symbol

API

CapEx

Cbench

$\mathrm{CPU}$

FIB

GC

GUI

HA

HT

IaaS

IETF

$\mathrm{I} / \mathrm{O}$

IoT

ISP

IT

JVM

KVM

NFV

ODL

OF

ONOS

ONF

OpEx
Definition

Application Programming Interface

Capital Expenditure

Controller Benchmarker

Central Processing Unit

Forwarding Information Base

Garbage Collection

Graphical User Interface

High Availability

Hyper-Threading

Infrastructure as a Service

Internet Engineering Task Force

Input/Output

Internet of Things

Internet Service Provider

Information Technology

Java Virtual Machine

Kernel-based Virtual Machine

Network Function Virtualization

OpenDaylight

OpenFlow

Open Network Operating System

Open Networking Foundation

Operational Expenditure 
OSGi

OSI

OVS

pCPU

PID

REST

SAL

SDN

$\mathrm{SSH}$

TCAM

TCP

UCS

UI

URL

$\mathrm{vCPU}$

$\mathrm{VM}$

XML

YANG
Open Service Gateway initiative

Open System Interconnection

Open vSwitch

Physical Central Processing Unit

Process Identifier

REpresentational State Transfer

Service Abstraction Layer

Software-Defined Networking

Secure Shell

Ternary Content-Addressable Memory

Transmission Control Protocol

Unified Computing System

User Interface

Uniform Resource Locator

Virtual Central Processing Unit

Virtual Machine

eXtensible Markup Language

Yet Another Next Generation 


\section{Chapter 1}

\section{Introduction}

The emergence of the Software-Defined Networking (SDN) paradigm has resulted in a revolution in computer networking. The separation of control plane and data plane in SDN improved flexibility, innovation, and scalability in network operation. Almost a decade ago, OpenFlow was introduced as a protocol for communication between the control plane (controller) and data plane (network devices). Since then, there have been many evolutionary improvements in this field. Today, $\mathrm{SDN}$ is mature enough to be deployed in production

networks. Thus, the number of telecom companies and enterprises adopting SDN is growing at a fast pace.

In a software-defined network, the controller acts as the "brain" of the network. Consequently, the performance of an SDN controller is of great importance. This thesis presents an experimental evaluation of two state-of-the-art OpenFlow controllers.

\subsection{Background}

Conventional networks are often complex, proprietary, inflexible, and expensive to operate. In a nutshell, they impede innovation rather than empowering it. When these types of networks become larger in scale, they get more complicated. Therefore, operating networks becomes a daunting task, requiring a lot of expertise and experience.

SDN simplifies network management by decoupling the control logic from the forwarding devices. This provides automation, programmability, and flexibility. SDN also accelerates innovation while significantly reducing the costs of building and operating a network.

SDN adoption is growing as its benefits become more apparent to organizations [71]. For instance, AT\&T plans to virtualize and control over $75 \%$ of its network with a software- 
defined architecture by 20201․ According to a report by Grand View Research $2^{2}$, the global SDN market is estimated to reach $\$ 70.41$ billion (USD) by 2024 .

As shown in Figure 1.1, the controller is an integral part of software-defined networks. In such networks, the applications and services enforce high-level policies on the underlying network via the controller. Based on those policies, the controller installs/uninstalls flow rules in the flow tables of network devices, behaves differently with each type of traffic, routes the traffic through certain paths, etc.

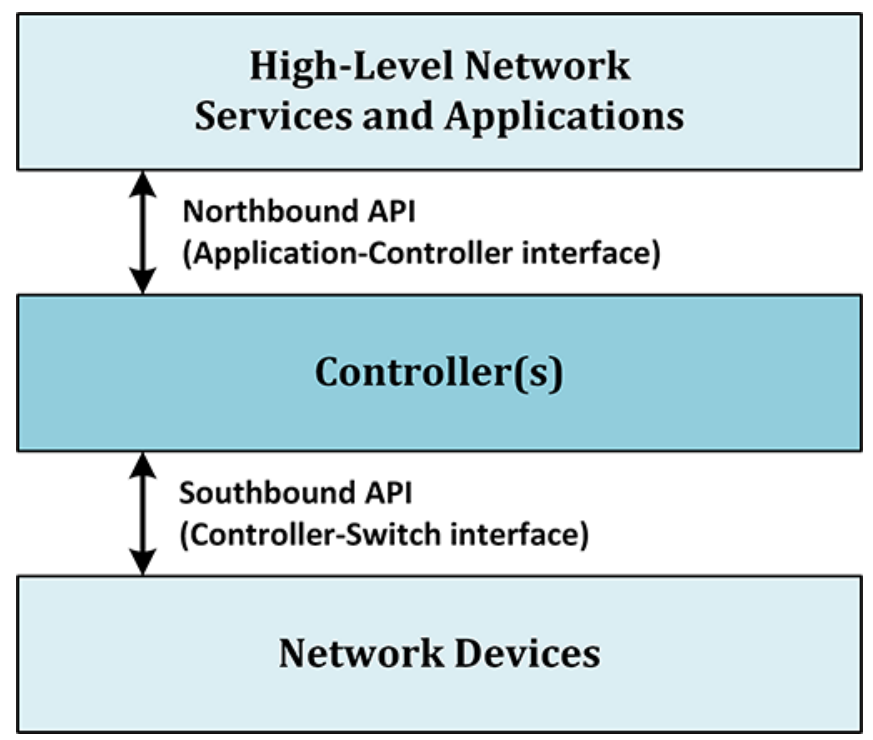

Figure 1.1: The role of a controller in software-defined networks $\mid 69$

Essentially there are two types of SDN controllers, namely centralized and distributed. With the emergence of SDN, centralized controllers (e.g., NOX [50], POX [20]) were developed and mostly used for the purpose of research or in small networks. However, since SDN matured and was deployed in production networks, distributed controllers were introduced, such as HyperFlow [89], Kandoo [53], Onix [61], ONOS [35], OpenDaylight [66], etc. The distributed controllers were developed to address the single-point-of-failure issue of the centralized controller, and to enable redundancy in the design of software-defined networks.

\footnotetext{
${ }^{1}$ http://about.att.com/innovation/sdn

2 http://www.grandviewresearch.com/press-release/global-software-defined-networking-sdn-market
} 
Among all open-source distributed controllers, ONOS and OpenDaylight are well-recognized since they are modular, support numerous features, and have a large technical community. OpenDaylight is the biggest SDN open-source project supported by the Linux Foundation, and has many industry partners and collaborators. On the other hand, ONOS is OpenDaylight's main competitor. ONOS is supported by the Open Networking Lab (ON.Lab) which is a non-profit association established by SDN pioneers and leaders from Stanford University and UC Berkeley. These two controllers are the primary candidates for deployment in enterprise-scale software-defined networks.

\subsection{Motivation}

Understanding the performance of the control plane in software-defined networks is crucial, since it impacts the performance of the entire network, applications, services, etc. The performance evaluation of SDN controllers can be carried out through benchmarking. OpenFlowbased controllers are usually benchmarked using Cbench [1] [90]. Benchmarking is a reliable and well-recognized approach for performance evaluation in many science and engineering disciplines. It is widely used in academia and industry to assess the performance characteristics of a given software platform. Also, it helps to identify performance bottlenecks and allows us to obtain an overall understanding of resource utilization of the desired system.

To the best of our knowledge, there is no recent study in the literature focusing on the performance evaluation of ONOS and OpenDaylight. Prior works have considered prototyping a new controller, or evaluated centralized OpenFlow controllers. ONOS and OpenDaylight have multiple releases and are mature enough in their development phase. Thus, to better

understand performance improvements (if any), it is important to reevaluate new releases of these controllers. That being said, the primary motivation of this research is to conduct an experimental evaluation of these controllers.

The second motivation of this study is to answer the following question; "How well do 
ONOS and OpenDaylight perform in virtualized environments?" In today's IT world, enterprises are more eager than ever to virtualize their infrastructure. This is mainly due to the numerous advantages of virtualization. That being said, virtualization puts application performance at risk. According to a survey by Gridstor $\mathrm{S}^{3}$ in 2013, improving the performance of applications for virtualized environments is the top business priority for mid-market organizations. Considering the importance of virtualization, we are motivated to study the performance of these controllers on virtual hosts.

The outcome of this research will be useful for the SDN community as well as other networking research groups, since there are many discussions on the performance of these two controllers.

\subsection{Objectives}

The primary objectives of this thesis are as follows:

1) Compare the performance of ONOS and OpenDaylight with each other, as well as other multi-threaded OpenFlow controllers.

2) Identify the impact of Hyper-Threading on performance of ONOS and OpenDaylight.

3) Evaluate the performance of ONOS and OpenDaylight on physical and virtual hosts.

\subsection{Contributions}

This thesis makes four primary contributions, listed as follows:

1) We construct a (dedicated) physical and virtual testbed for experimental evaluation of ONOS and OpenDaylight.

\footnotetext{
$\sqrt[3]{\text { https://apmdigest.com/application-performance-in-virtualized-environments }}$
} 
2) We identify and compare the performance of ONOS (Goldeneye version) and OpenDaylight (Beryllium-SR2 version) on the physical and virtual hosts. This includes discovering the impact of Hyper-Threading and Virtualization on the performance of these controllers. We also compare our results with prior work to better understand the performance differences (if any) between different releases of these controllers.

3) We identify issues with memory management and CPU utilization for ONOS and OpenDaylight in the throughput test.

4) We improve thread scalability of ONOS and OpenDaylight by reducing inter-socket communication overhead.

\subsection{Thesis Organization}

The rest of this thesis is organized as follows. Chapter 2 provides an overview of related technologies and platforms, including Software-defined Networking, OpenFlow, ONOS controller, OpenDaylight controller, virtualization, and Hyper-Threading. It also reviews prior studies on performance evaluation of OpenFlow controllers. Chapter 3 discusses our benchmarking methodology and the testbed. Also, it describes the benchmarking tool used to evaluate the controllers. Chapter 4 analyzes baseline results for throughput, latency, and thread scalability tests. Chapter 5 examines memory usage and CPU utilization of both controllers in throughput tests. It also discusses the results of our follow-up tests on thread scalability of

controllers when threads reside on one socket. Lastly, Chapter 6 presents conclusions and outlines directions for future work. 


\section{Chapter 2}

\section{Background and Related Work}

\subsection{Overview}

In this chapter, we provide the necessary background regarding the technologies and platforms that are involved in this study. Section 2.2 provides the background on Softwaredefined Networking, including architecture, layers, and key concepts. Section 2.3 reviews OpenFlow's principal components, communication messages, modes of operation, and types of OpenFlow controllers. Section 2.4 and Section 2.5 introduce ONOS and OpenDaylight controllers, respectively. In Section 2.6, we review Virtualization technology, including different types, and solutions. Section 2.7 summarizes Hyper-Threading technology and briefly reviews the literature on the impact of Hyper-Threading on performance. Section 2.8 discusses related work on the performance of OpenFlow controllers. Section 2.9 summarizes the chapter.

\subsection{Software-Defined Networking (SDN)}

In a simple definition, computer networks comprise end hosts that connect to each other. The network devices and middleboxes that connect these end hosts could be switches, routers, and firewalls. Usually, these devices run proprietary operating systems.

The variety of operating systems in network devices adds an extra layer of complexity to the network operation. Besides, the multi-vendor environments require a high level of expertise. Hence, the network administrators need to have vast knowledge of different configuration interfaces, features, and commands that often vary across vendors and even across different products from the same vendor [47]. Furthermore, to apply any change in the net- 
work, the network administrator needs to login to each device and modify the configuration. In a large network, this procedure creates a significant administrative overhead. As a result, network operation has become a challenging and error-prone task 69].

These challenges led to the idea of Software-Defined Networking (SDN), which is a new approach to designing networks. As shown in Figure 2.1, SDN decouples the control plane from the data plane. Specifically, SDN moves the control logic off of packet processing devices and onto external programmable controllers [54]. The control plane can be described as the "brain" of the network. The control plane decides how to handle each type of traffic. The data plane (also known as the forwarding plane) is a simple device to forward the traffic based on the decision of control plane.

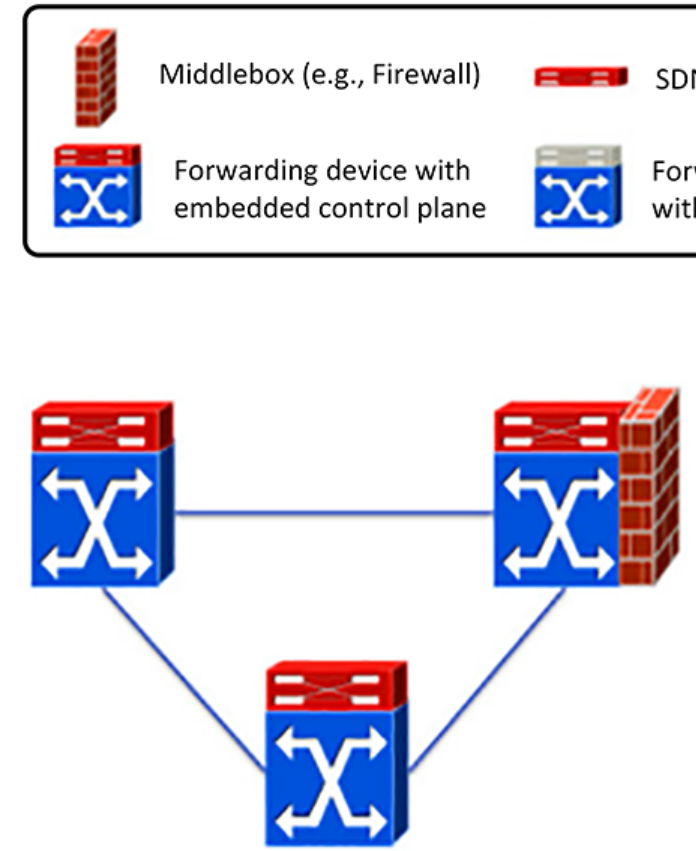

Traditional Network
SDN Controller

Forwarding device without control plane

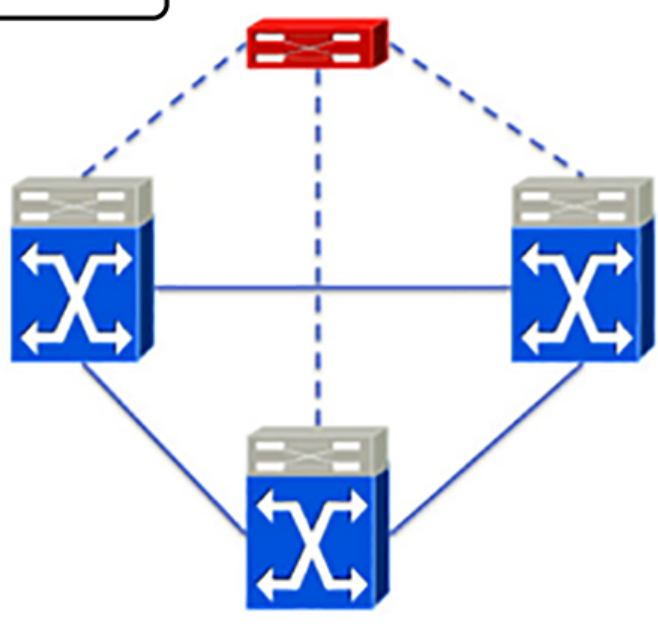

Software-Defined Network

Figure 2.1: Traditional network architecture versus Software-Defined Network |69

In the SDN architecture, the controller is the control plane, and manages the data planes that carry the network traffic. The migration of the control plane, which used to be tightly integrated with network devices in conventional networks, into open and logically central- 
ized controllers, allows the underlying infrastructure to be abstracted from the point of view of applications. Furthermore, decoupling the control plane paves the way for a more programmable, flexible, vendor-agnostic, cost effective, and innovative network architecture [31]. Software-Defined Networking facilitates design, implementation, troubleshooting, and management of the network. In essence, SDN provides full end-to-end visibility of the network, and overcomes the limitations of proprietary platforms and network operating systems.

It is worth noting that Software-Defined Networking did not pioneer the idea of programmable networks. The idea of separating the control plane from the data plane was first introduced in the 4D project [49]. The goal of 4D was managing the network infrastructure. The Ethane [39] and SANE [40] projects provided a finer granularity of control (i.e., flow-based rather than FIB-based control). The Ethane architecture consisted of two components, namely controller and switch. The centralized controller manages the policies and flow table(s) used by Ethane switches in the network. Also, Forwarding and Control Element Separation (ForCES) [97] was proposed by the IETF. They introduced a similar approach to Ethane, with control plane and data plane separation.

\subsubsection{SDN Architecture}

SDN layers and architecture is defined in IETF RFC 7426 [51]. Figure 2.2 illustrates the logical layers and architecture of SDN. Network devices reside in the bottom layer, which is called the "Infrastructure Layer". The middle layer, with the network operating system, is called the "Control Layer". The SDN controllers work in this layer. The communication between devices in the Infrastructure Layer and the controller(s) in the middle layer is through an open interface called the Southbound API. The very top layer in SDN architecture is called the "Application Layer", and is devoted to applications that define high-level policies for network behavior. The communication between these application and SDN controllers occurs via another type of open interface called the Northbound API. We will review the components of the SDN architecture in a bottom-up approach. 


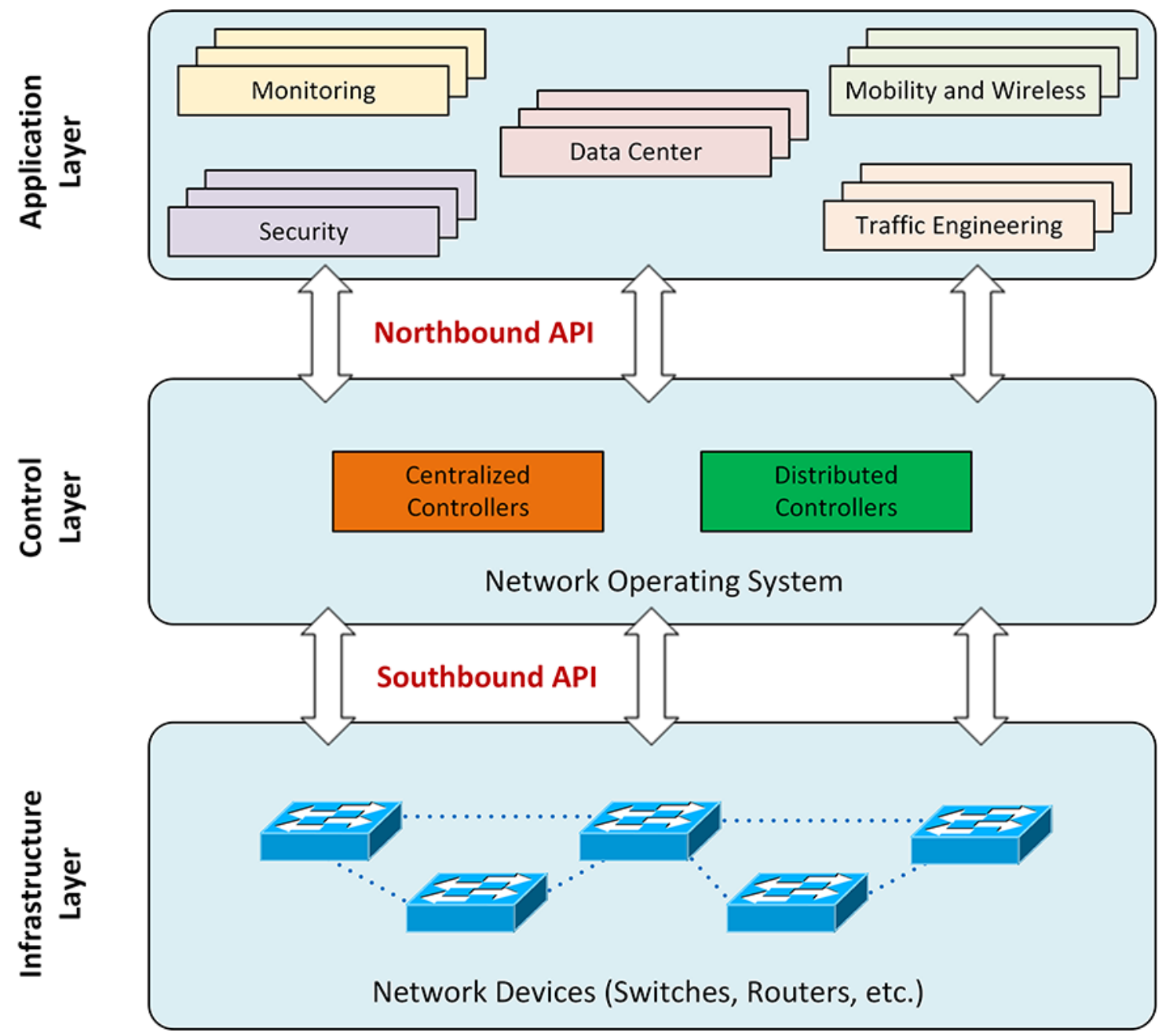

Figure 2.2: SDN high-level architecture 57

\subsubsection{Infrastructure Layer}

The infrastructure layer hosts all forwarding devices. The infrastructure may include different network equipment, such as routers, physical or virtual switches, wireless access points, etc. In a software-defined network, such devices are typically basic forwarding hardware, and are accessible via an open interface (API). The control logic of these devices are off-loaded to a remote controller. In SDN terminology, forwarding devices are typically referred to as switches. 


\subsubsection{Southbound API}

An important aspect of SDN is the linkage between the control plane and the data plane. The Southbound API (also known as the Southbound interface) is the language for communication between controller and forwarding devices (e.g., switches, routers). Since this interface controls forwarding devices, its reliability and performance are of high importance. The reason that this interface is called "Southbound" is that in the schematic layered architecture of SDN, with the controller positioned in the central part, the forwarding devices are positioned below the controller. Hence, the controller speaks to downstream or Southbound nodes.

To date, OpenFlow is the most broadly deployed standard Southbound interface for SDN 62. Hence, the majority of controllers only support OpenFlow for the Southbound interface. Since we evaluate the performance of two OpenFlow controllers in this study, we discuss OpenFlow in further detail in Section 2.2. One feature that makes OpenFlow attractive is that OpenFlow places the forwarding rules and instructions directly into TCAM or FIB on the switch. Most other Southbound APIs need to talk to the operating system on the local device [64].

Even though OpenFlow is the most well-known Southbound API, there are other Southbound interfaces for deployment in SDN networks. The other Southbound APIs and protocol plugins in alphabetical order are: BGP [77], Hardware Abstraction Layer (HAL) 34 [73], LISP [46], NetConf [43], OpenState [36], Open vSwitch Database (OVSDB) [75], OpFlex [86], PCEP [93], POF 21] [88, Programmable Abstraction of Datapath (PAD) 33], SNMP [52]. Among these APIs, NetConf is more common than the others, because it is widely supported by network devices from different vendors. Hence, we provide a brief introduction to NetConf.

In 2006, the IETF proposed the Network Configuration Protocol (NetConf) 43 in RFC 6241, as a protocol to manage network devices and modify their configuration. NetConf 
uses an XML-based API for configuration and protocol messages. Also, it uses a Remote Procedure Call (RPC) model for communication between the NetConf client and NetConf server. The client encodes RPC in XML format and sends it over a Secure Shell (SSH) session, and the server responds with the same format. NetConf employs the YANG [37] data modeling language to specify the data models and structure. NetConf allows the network administrator to test and validate the configuration before applying it to the device. However, NetConf does not provide separation of the control plane from the data plane; control logic still resides on network devices.

\subsubsection{Control Layer}

The control layer is the cornerstone of SDN, since it connects the SDN applications to the network devices. It is responsible for configuring and managing the forwarding devices via the Southbound API. The controller decouples the control logic of forwarding devices, and has been compared to an operating system [50]. It provides a global view of the network that facilitates network control and enables the SDN applications to control the network and offer new functionalities.

The SDN controllers are grouped into two categories, namely centralized and distributed. The centralized controllers were developed in the initial phase of SDN, and were mostly targeted for campus networks and research purposes. Centralized controllers suffer from two main problems. First, they can become a single point of failure (SPOF) for the entire network, since there is no redundancy. Second, a single controller has a limited capacity, and cannot deal with a large volume of traffic. SDN technologies need to support carrier-grade and large-scale networks, such as data centers and service provider networks. Considering the mission-critical nature of these types of networks, SDN controllers are now designed to be distributed, offering higher availability and performance. Table 2.1 summarizes the current well-known SDN controllers. 
Table 2.1: Overview of well-known SDN controllers |32]| 62 | 669

\begin{tabular}{|c|c|c|c|c|c|}
\hline Name & Architecture & $\begin{array}{l}\text { Multi- } \\
\text { Thread } \\
\text { Support }\end{array}$ & $\begin{array}{c}\text { Programming } \\
\text { Language }\end{array}$ & Year & Developer \\
\hline Beacon & Centralized & Yes & Java & 2011 & Stanford University \\
\hline Floodlight & Centralized & Yes & Java & 2012 & Big Switch Networks \\
\hline HyperFlow & Distributed & Yes & $\mathrm{C}++$ & 2010 & University of Toronto \\
\hline IRIS & Centralized & Yes & $\mathrm{C}$ & 2014 & ETRI \\
\hline Kandoo & Distributed & Yes & $\mathrm{C}, \mathrm{C}++$, Python & 2012 & University of Toronto \\
\hline Maestro & Centralized & Yes & Java & 2010 & Rice University \\
\hline MUL & Centralized & Yes & $\mathrm{C}$ & 2013 & KulCloud \\
\hline NOX & Centralized & No & $\mathrm{C}++$ & 2008 & Nicria \\
\hline NOX-MT & Centralized & Yes & $\mathrm{C}++$ & 2012 & University of Toronto \\
\hline Onix & Distributed & Yes & C, Python & 2010 & Stanford University \\
\hline ONOS & Distributed & Yes & Java & 2014 & ON.Lab \\
\hline OpenDaylight & Distributed & Yes & Java & 2014 & Linux Foundation \\
\hline POX & Centralized & No & Python & 2014 & Nicira \\
\hline Ryu & Centralized & No & Python & 2011 & NTT \\
\hline Runos & Distributed & Yes & $\mathrm{C}++$ & 2015 & ARCCN \\
\hline Trema & Centralized & Yes & C, Ruby & 2011 & NEC \\
\hline
\end{tabular}

\subsubsection{Northbound API}

The Northbound API (also known as the Northbound interface) is the programming interface that enables the top layer of a software-defined infrastructure to program the controller, and subsequently the underlying network. Essentially, it is a RESTfu ${ }^{1}$ application programming

\footnotetext{
${ }^{1}$ REST (REpresentational State Transfer) is a Web architectural method that allows two pieces of software communicate with each other. REST structure is quite similar to client-server communications, and it
} 
interface (API) that runs on the controller and hosts software applications. The Northbound API can be written by network operators, service providers, and researchers to deploy desired applications, such as advanced path computation algorithms, and loop avoidance mechanisms on the network. In essence, this interface, rather than directly talking to network devices, expresses the high-level behavior of the network to the controller. This shifts the focus from the infrastructure to the application. Standardizing an API for SDN Northbound applications has been an ongoing debate since the emergence of SDN [10]. However, there is no standard for the Northbound API yet.

\subsubsection{Applications and Services}

The application layer embodies all SDN applications through which high-level policies and network behavior can be defined and managed. Network applications implement the control logic that is translated into commands/rules to be installed in the data plane of the network devices. They dictate the behavior of the forwarding devices. For instance, a traffic engineering application defines the path through which delay-sensitive traffic flows from host A to host B. Based on the topology of the network, the routing application finds the best path, and instructs the controller to install the respective forwarding rules in related forwarding devices. In general, the SDN applications can be classified into five categories, namely traffic engineering, mobility and wireless, measurement and monitoring, security, and data center networking 62].

uses the HTTP protocol 78 . 


\subsection{OpenFlow}

In this section, we provide a summary of OpenFlow and review its architecture. Also, we explain OpenFlow's communication messages, modes of operation, and types of OpenFlow controllers.

McKeown et al. [65] at Stanford University first proposed OpenFlow in 2008. They introduced it as a means for networking researchers to run experiments on campus networks and large-scale testbeds like GENI ${ }^{2}$. Their vision at the time was the deployment of OpenFlow on real-world networks, and encouraging networking vendors to support OpenFlow in their future switching products. The authors stated that OpenFlow is a "pragmatic compromise", which allows researchers to evaluate their ideas on a set of switches in a consistent way, without requiring vendors to disclose the internal architecture of their switches. In the early days, there was a misconception that SDN and OpenFlow are equivalent, which was mainly because SDN was created after the introduction of OpenFlow 45].

Currently, OpenFlow is maintained by the Open Networking Foundation $(\mathrm{ONF})^{3}$. ONF is a non-profit organization founded in 2011, with a goal to accelerate the adoption of SDN.

As shown in Figure 2.3, the OpenFlow architecture has three main components: OpenFlow controller, OpenFlow-compatible device (e.g., OpenFlow switch), and a secure channel [12 [15] 63]. The OpenFlow controller is software that provisions and manipulates the flow tables of OpenFlow devices. The OpenFlow controller uses TCP port 6633 to communicate with devices. Typically, the connection between controller and forwarding devices is encrypted using Secure Socket Layer (SSL) or Transport Layer Security (TLS). Through this secure channel, the controller manages the devices, receives events from them, and pushes the configuration to devices. An OpenFlow-compatible device is capable of forwarding packets according to the rules defined in the flow table(s).

\footnotetext{
${ }^{2}$ Global Environment for Network Innovations, http://www.geni.net

$\sqrt[3]{\text { http: //www.opennetworking.org }}$
} 


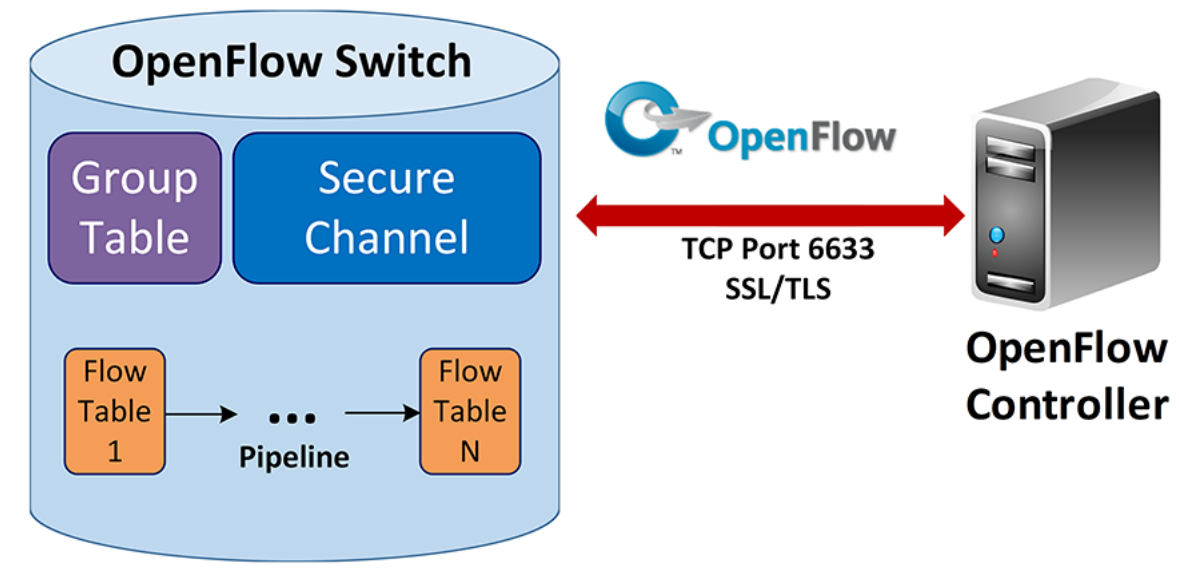

Figure 2.3: OpenFlow components 29

OpenFlow benefits from the fact that most network devices have flow tables for essential networking functions. In the OpenFlow architecture, the forwarding device (e.g., OpenFlow switch) contains one or more flow tables, a group table, and a secure channel to the controller. Each flow table includes a set of flow entries. A flow entry typically has three fields, namely header fields, actions, and counters. The header fields are used to match against incoming packets. As shown in Figure 2.4, the header fields consist of ingress port, MAC addresses of source and destination, IP addresses of source and destination, VLAN ID, source and destination TCP or UDP ports, and optionally metadata specified by a previous table. The actions are instructions to be applied when a match occurs; they dictate how to handle such packets (e.g., the action might be to forward a packet via a specified port). The Counters collect statistics for the particular flow, such as the number of received packets, the number of bytes, and duration of the flow [30] [69]. Aside from these three components, a flow entry may have other fields, such as priority, timeout, and cookies.

As shown in Figure 2.3, the OpenFlow pipeline in each OpenFlow switch may contain multiple flow tables. The OpenFlow pipeline processing outlines how packets are associated with flow tables. An OpenFlow switch must have at least one flow table, and may optionally have more flow tables. The Group table provides the ability to add support for port group 
abstraction for multi-pathing, which allows OpenFlow to represent a set of ports as a single entity for forwarding packets.

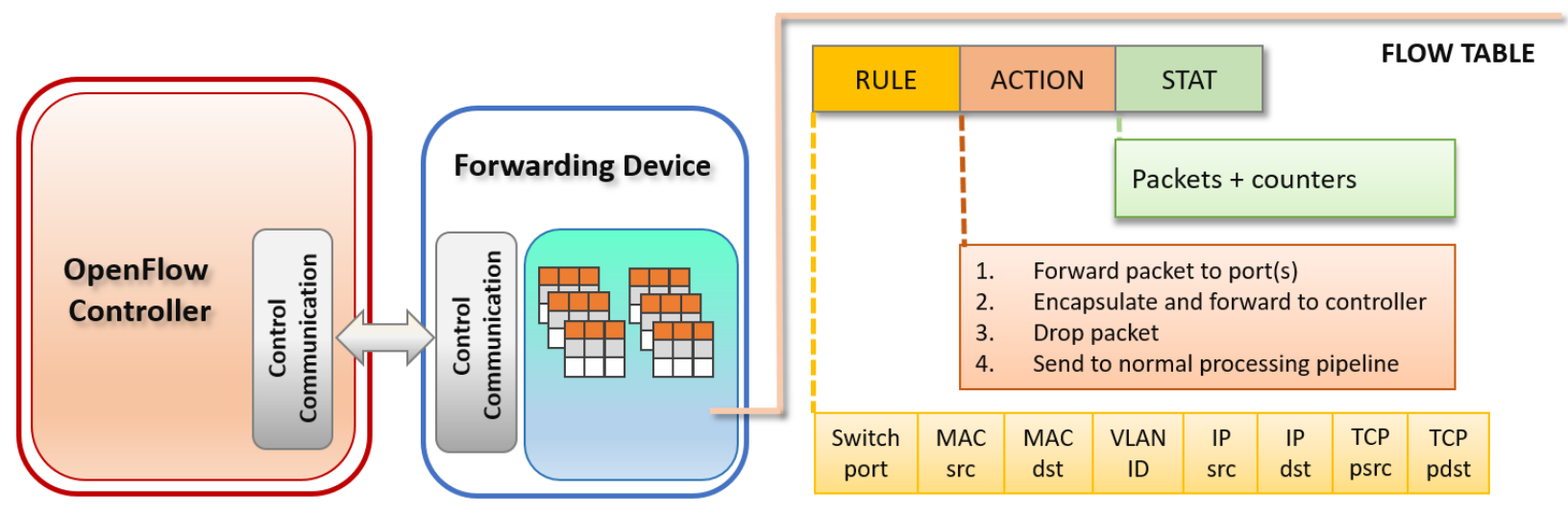

Figure 2.4: Schematic view of OpenFlow device and OpenFlow table 62

Figure 2.5 shows the packet flow structure in an OpenFlow switch. In the OpenFlow device, upon arrival of a packet from a new flow, the lookup procedure begins in the first table and ends either with a match in one of the tables of the pipeline or with a miss (indicates no rule is found for that packet). A flow rule can be defined by combining different matching fields. If there is no default rule, then the switch discards the packet. However, typically there is a default rule that tells the switch to encapsulate and send the packet to the controller if there is no match. The priority of the rules follows the sequence of the tables and the row order in a flow table. The possible actions are:

1) Forward the packet to outgoing port(s)

2) Encapsulate the packet and send it to the controller

3) Drop the packet (no match found, and no default action defined for unmatched packets)

4) Send the packet to the next flow table or the special tables (e.g., group or metering tables introduced in the latest OpenFlow protocol) 


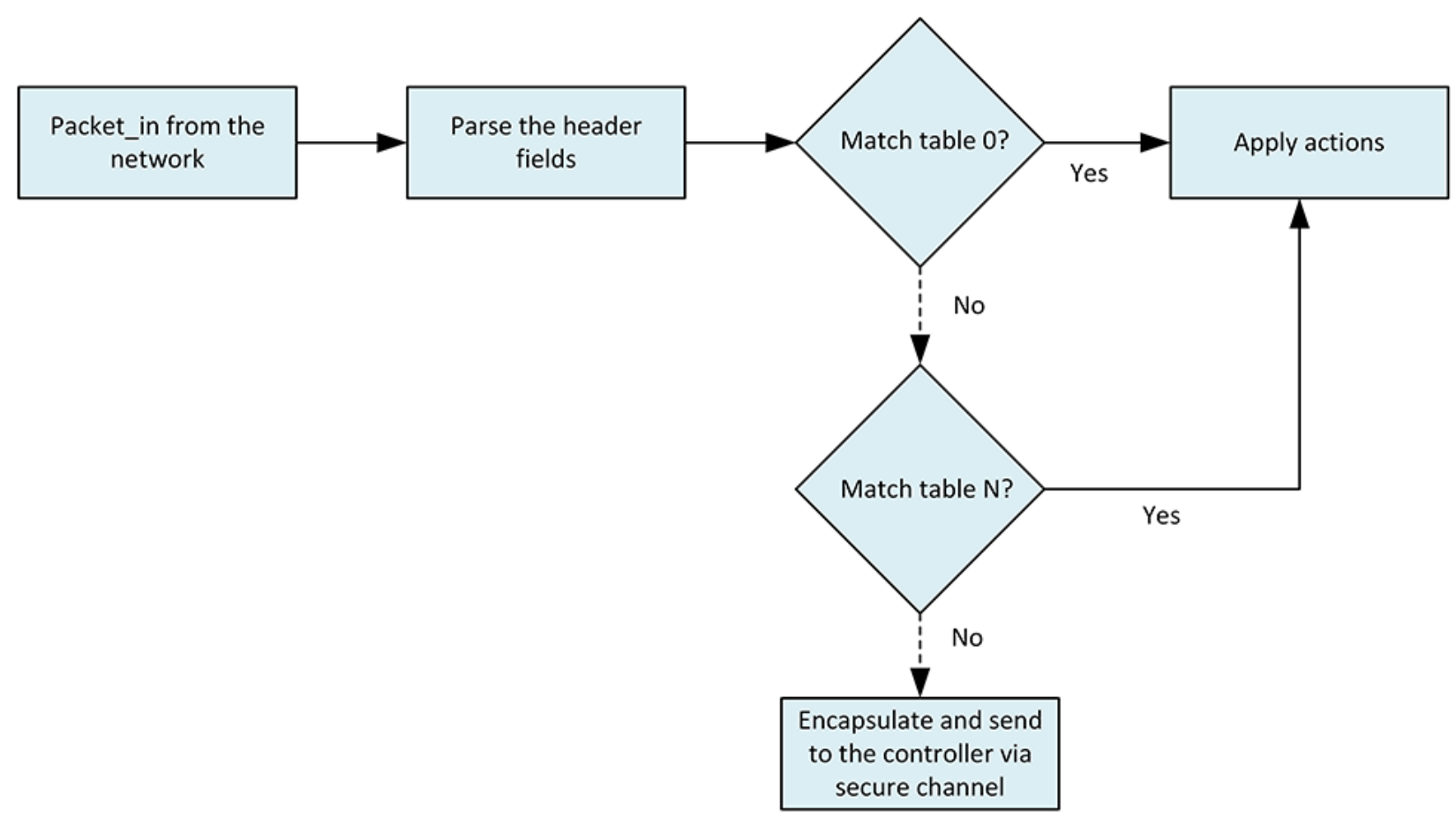

Figure 2.5: Packet flow structure in the OpenFlow switch 29

OpenFlow has six different versions, which are documented in the OpenFlow Switch Specification. OpenFlow version 1.0 was the first release of OpenFlow, published by Stanford University in 2008. Since 2011, the Open Networking Foundation (ONF) has been maintaining the OpenFlow switch specification. Version 1.0 is currently broadly adopted by OpenFlow vendors. In that version, forwarding relies on a single flow table, and matching focuses only on layer 2 information and IPv4. Version 1.1 supports multiple flow tables and MPLS tags. OpenFlow version 1.2 supports a more flexible matching structure and also supports IPv6. Version 1.3 added the support for multiple parallel channels between switches and controllers. Version 1.4 was published in 2013. The main change in that version is the improvement of OpenFlow extensible match, which was introduced in version 1.2. This improvement gives more flexible classification capabilities to the user to match packet header fields and classify flows. This version also supports optical ports. The latest version of OpenFlow is version 1.5. This version was published in 2014, and introduced egress ta- 
bles, which enable packet matching based on output port [45] [62]. Table 2.2 summarizes the major changes in OpenFlows versions.

Table 2.2: OpenFlow major changes in each release $[42]$

\begin{tabular}{|c|c|c|}
\hline Version & Major Feature & Reason \\
\hline \multirow{3}{*}{$1.0-1.1$} & Multiple flow tables & Avoid flow entry explosion \\
\cline { 2 - 3 } & Group table & Enable applying action set to group of flows \\
\cline { 2 - 3 } $1.1-1.2$ & Full VLAN and MPLS support & Enable integration with non-OpenFlow network \\
\cline { 2 - 3 } & Oultiple controller & Extend matching flexibility \\
\hline \multirow{3}{*}{$1.2-1.3$} & Meter table & HA / Load balancing / Scalability \\
\cline { 2 - 3 } & Table miss entry & Add QoS and DiffServ capability \\
\hline \multirow{3}{*}{$1.3-1.4$} & Synchronized table & Provide flexibility \\
\cline { 2 - 3 } & Bundle & Enhance table scalability \\
\hline \multirow{3}{*}{$1.4-1.5$} & Egress table & Enhance switch synchronization \\
\cline { 2 - 3 } & Scheduled bundle & Further enhance switch synchronization \\
\hline
\end{tabular}

\subsubsection{OpenFlow Messages}

The OpenFlow protocol has three message types, namely controller-to-switch, asynchronous, and symmetric. Each message type has multiple sub-types [30]. The main message types and their sub-types are described below:

1) Controller-to-switch: As the name indicates, the controller initiates this type of message, which is used to manage or inspect the state of the switch. The controllerto-switch message may or may not require a response from the switch. The six main controller-to-switch message types are described below:

- Features: Upon OpenFlow channel establishment between the controller and 
the switch, the controller sends a feature_request message to the switch asking for its identity and basic capabilities. The switch must respond with a feature_reply message that indicates the supported capabilities.

- Configuration: The configuration messages are sent by the controller to set and query the configuration parameters in the switch. The switch only responds to a query from the controller.

- Modify-State: The controller sends this message to manage the state of the switches. The purpose of this message is to modify flow tables (add/remove flow), modify actions, and set switch port settings.

- Read-State: The controller sends this message to collect information from the switch, such as current configuration, statistics, etc.

- Packet_out: The controller uses this message to send packets out of the desired port(s) on the switch, and to forward the packets received via Packet_in messages. This message typically contains the list of actions to be applied to the packet (in the order specified). An empty list of actions results in the switch dropping the packet. The packet-out message must embody a buffer ID referencing a packet stored in the switch or the full packet.

- Barrier: The controller sends a Barrier Request message to ask the switch to complete processing of all messages sent before receiving this message. This message is used by the controller to ensure that the switch processes all message dependencies and sends all notifications for completed operations before sending new requests.

- Role-Request: This controller sends this message to set its Controller ID, the role of its OpenFlow channel, and query either of these parameters. This message is useful when the switch has a connection to more than one controller. 
2) Asynchronous Messages: These messages are initiated by the switch without the request of the controller. This message type is used to update the controller about network events or changes in the state of the switch. The primary asynchronous message types are described below:

- Packet_in: The switch sends a packet_in message to the controller when it receives a packet for which it does not have a match in its flow table, or the packet matches an action that requests the switch to send it to the controller. If the switch has enough memory to buffer the packet, it only sends some portion of the packet header to the controller. If the packet is buffered, then the packet_in message includes 128 bytes from the original packet with the default setting. The controller typically processes the buffered packets with a Packet_out or Flow_mod message, or they automatically expire when their timeout is exceeded. If the switches don't have enough memory, or are configured not to buffer packets, they send the full packet to the controller.

- Flow-Removed: The switch informs the controller about the removal of a flow entry from the flow table with this message. This message could be generated due to a controller's flow deletion request, or flow expiry (when the flow timeout is exceeded).

- Port-status: This message is used by the switch to update the controller about a port state change (e.g., if a port was shut down manually by the user, or when the port configuration changes).

- Role-status: The switch generates this message to inform the controller about a change of its role. For instance, when a new controller becomes the master, the switch notifies the former controller regarding this role change with the role-status message.

- Controller-status: This message is used by the switch to inform the controller 
when the status of the OpenFlow channel changes. The switch sends this message to all controllers (if more than one), to assist in the failover procedure in case the controllers lose the ability to communicate with each other.

- Flow-monitor: With this message, the switch informs the controller regarding a change in its flow table. The controller may define a few monitors to track the status of the flow tables in the switch.

3) Symmetric Messages: These type of messages are sent by either the controller or the switch. The primary symmetric message types are described below:

- Hello: Upon connection initiation, the controller and switch exchange hello messages.

- Echo: This message is sent bidirectionally. The sender initiates with echo request, and the other side responds with an echo reply. It is used to verify the liveness of the controller-switch connection. Also, it may be used to measure the bandwidth or the latency of the link.

- Error: This message is used by either the switch or the controller to notify the other side regarding a problem. It is typically used by the switch to indicate the failure of a request sent by the controller.

- Experimenter: This type of Symmetric message was also known as Vendor message in the previous OpenFlow versions. It is used by OpenFlow switches to offer additional functionality within the standard OpenFlow message types. In future versions, it may also be used to provide new features.

\subsubsection{OpenFlow Controller Modes of Operation}

The OpenFlow controller has two different modes of operation, namely reactive and proactive.

In reactive mode, forwarding devices (e.g., OpenFlow switches) need to consult with the 
controller to make a decision about a new flow. Hence, when the first packet of a new flow arrives at the switch, it triggers the controller to install associated flow entries in the OpenFlow switches. This results in a short delay for the new traffic flow, since the first packet of each flow is forwarded to the controller for the decision (e.g., drop or forward). However, the next packets within the same flow are forwarded at the line rate of the forwarding device. This mode makes efficient use of the flow table's memory. However, it has an additional setup time for every new flow, and if the switch loses its connection with the controller, it has very limited functionality [48] [57].

In proactive mode, flow rules are pre-populated in the flow tables of switches. That is, the flow setup happens before receiving the first packet of the new flow. The main advantage of the proactive mode is that it has lower flow setup latency for the first packet in a flow, and it reduces the number of times that switches need to consult with the controller.

\subsection{ONOS}

Open Network Operating System (ONOS) [11] [35] is an open-source Java-based carrier-grade SDN controller. It is targeted for mission-critical networks such as Internet Service Providers. ONOS is developed by the Open Networking Lab (ON.Lab) in concert with leading service providers (AT\&T, NTT Communications), and vendors (Cisco, Ciena, Ericsson, etc.). ONOS released its first version named Avocet in December 2014. ONOS is designed to provide high availability, high performance, and scalability.

As a distributed SDN controller, ONOS can be deployed on a cluster of servers. The network operator can add ONOS instances without disruption, while all the services and applications remain functional. Even though the ONOS core is distributed to provide high availability, ONOS is logically centralized, and the separate instances in the ONOS architecture can be viewed and accessed as a single system. Network devices and applications do not know whether they are working with a single-node controller or multiple instances. 
This enables service providers to scale their control plane capacity seamlessly without any disruption. Figure 2.6 illustrates the high-level architecture of ONOS controller.

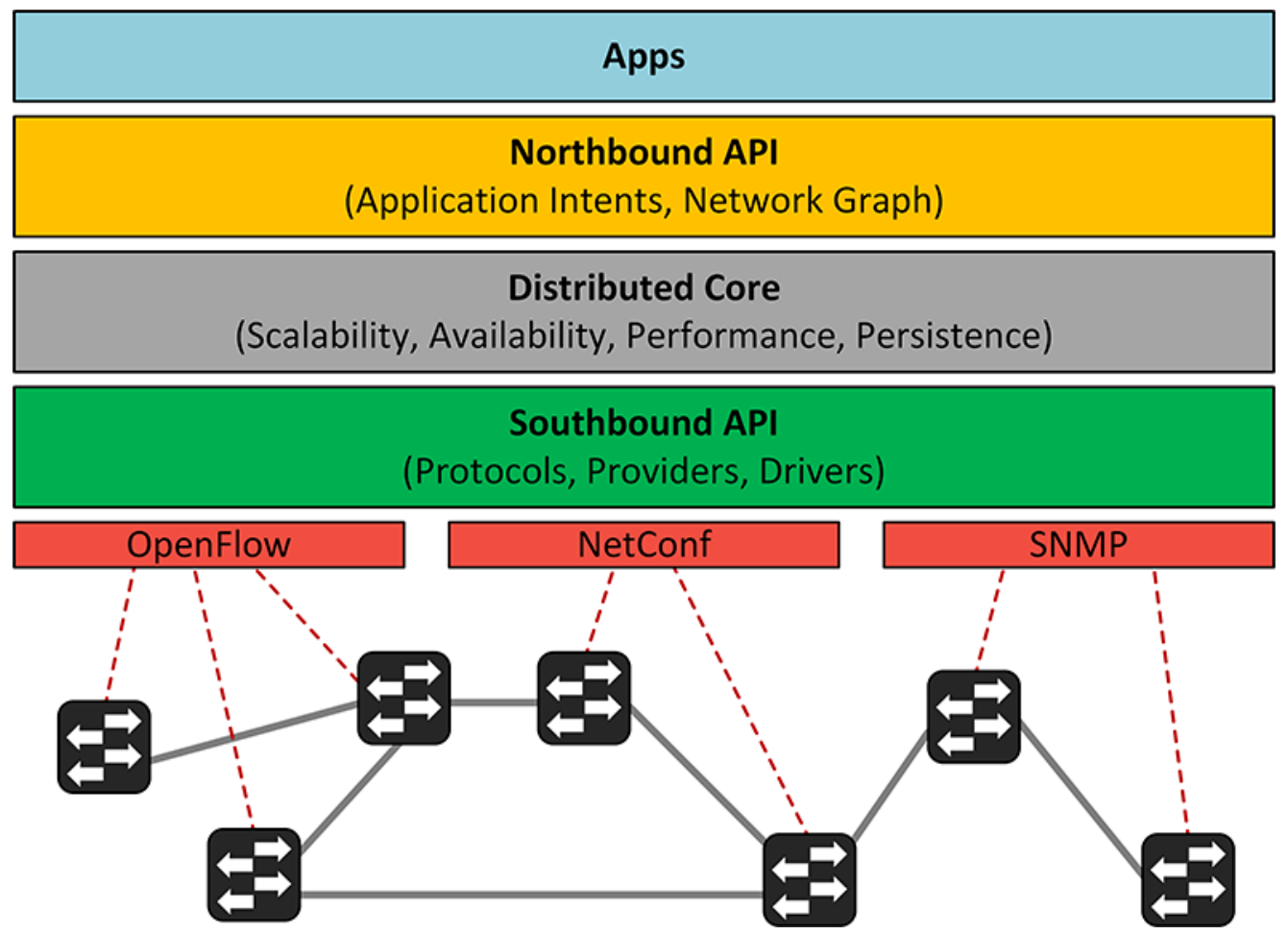

Figure 2.6: ONOS high-level architecture 11

For Northbound interactions, ONOS employs two types of Northbound abstractions, namely Intent Framework and Global Network View. The Intent Framework enables the application to request a service from the network without knowing the details of the deployment. The intents are translated into instructions for network devices. For example, an intent to create a path between host $\mathrm{A}$ and host $\mathrm{B}$ is translated into two objectives that each provide one direction of flow. The objectives then are compiled into instructions that are sent to network devices. On the other hand, the Global Network View provides a view of the network to the application (hosts, switches, links). The application can program the network based on the network topology through supported APIs.

ONOS interacts with the underlying network through its Providers. Providers are ONOS applications based on OSGi components. These applications can be dynamically enabled or 
disabled at run time. The primary purpose of providers is to abstract the configuration, control, and management operations of a specific family of devices (e.g. OpenFlow, Netconf, etc.).

Following are some of the primary use cases of ONOS [11]:

- Multi-layer network control in multi-vendor environment

- Migrating to SDN with the SDN-IP

- Segment routing, evolving, and improving MPLS network

- Multicast content distribution

\subsection{OpenDaylight}

OpenDaylight [14] [66] is an open-source Java-based distributed SDN controller for networks of any size and scale. The Linux Foundation has had a pivotal role in the development of OpenDaylight. However, a consortium of tech companies (e.g., Cisco, Brocade, Red Hat, HP) contributed to its development. The OpenDaylight project was started in April 2013, and announced its first release named Hydrogen in February 2014. It is mostly targeted for data center environments rather than service provider networks. OpenDaylight is built on Model-Driven Software Engineering principles. It supports OpenFlow and other network programming technologies. OpenDaylight works with the majority of Southbound APIs and supports numerous vendor-specific protocol plugins such as LISP, NetConf, BGP, OVSDB, SNMP, PCEP, etc. Figure 2.7 presents the high-level architecture of the OpenDaylight controller. 

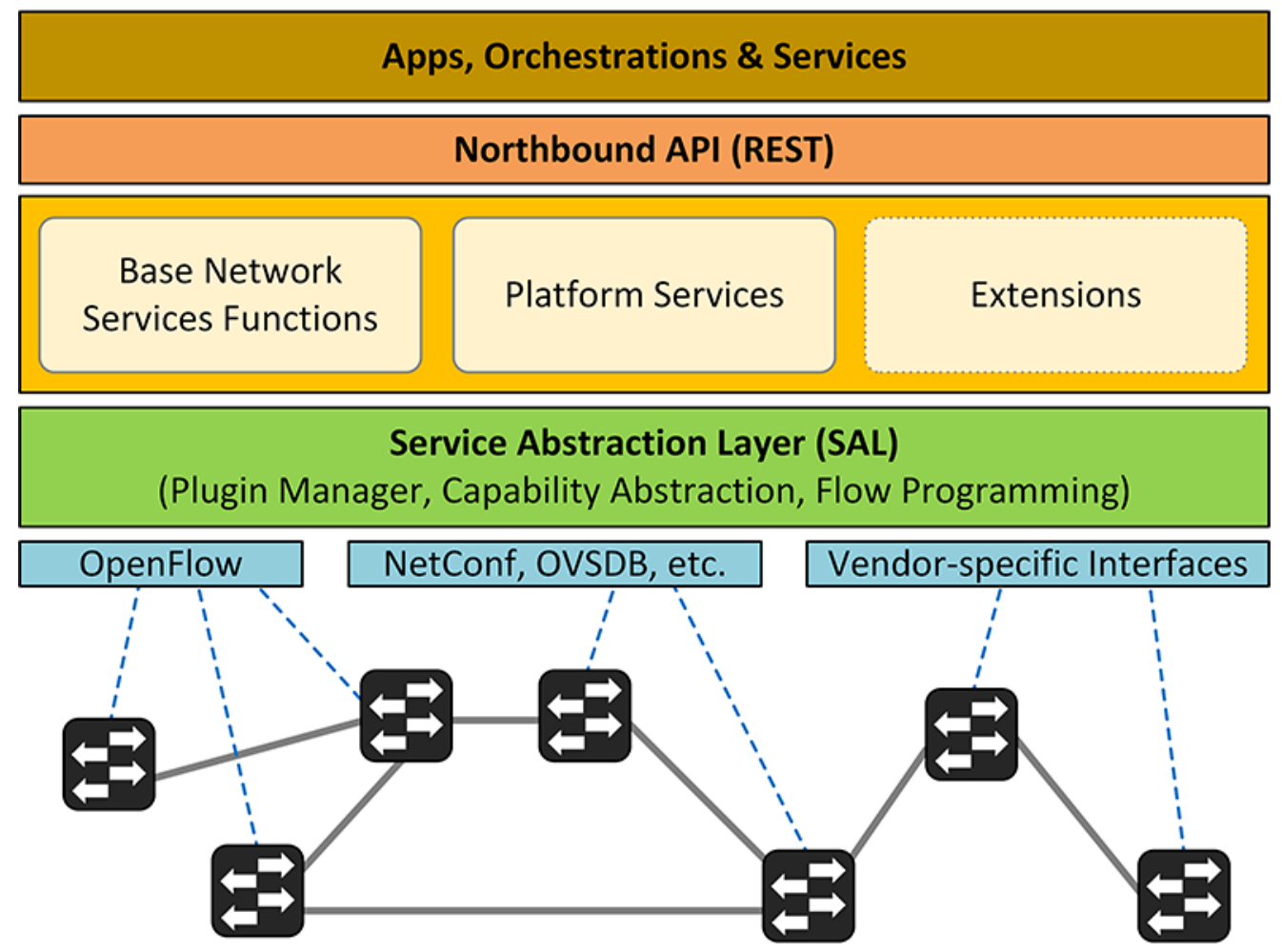

Figure 2.7: OpenDaylight high-level architecture 14

OpenDaylight embodies a Service Abstraction Layer (SAL), which connects protocol plugins and Service Network Function modules. Initially, OpenDaylight was using an APIDriven SAL (AD-SAL) approach, but now uses Model-Driven SAL (MD-SAL), since AD-SAL was ineffective. MD-SAL provides a standard approach to plugin development and enables unification between Northbound and Southbound APIs. MD-SAL uses YANG as a modeling language for describing all network services. YANG makes it easy to develop core modules, platform components, and the Northbound applications by providing built-in structures and types, such as lists and containers.

Following are some of the primary use cases of OpenDaylight [14]:

- Automated service delivery

- Network resources optimization

- Agile service delivery on the Cloud and NFV infrastructure 


\subsection{Virtualization}

Virtualization technology was introduced by IBM in the late 1960s. At the time, powerful and costly mainframe computers were underutilized. Thus, to use the maximum capabilities of their resources, techniques such as time-sharing and multiprogramming were developed. These techniques formed the foundation of virtualization [84].

Virtualization refers to a technology that provides a layer of abstraction (often called hypervisor) between the physical hardware and the operating system. This abstraction provides flexibility, and facilitates system resource sharing, and management. That being said, virtualization hides the characteristics of the hardware from the operating systems, applications, and users. This enables abstraction of operating system or application from the underlying infrastructure. Virtualization is the cornerstone of some other technologies such as Cloud Computing, SDN, Network Function Virtualization (NFV), etc.

Figure 2.8 illustrates the concept of virtualization and compares the traditional and virtual architecture.

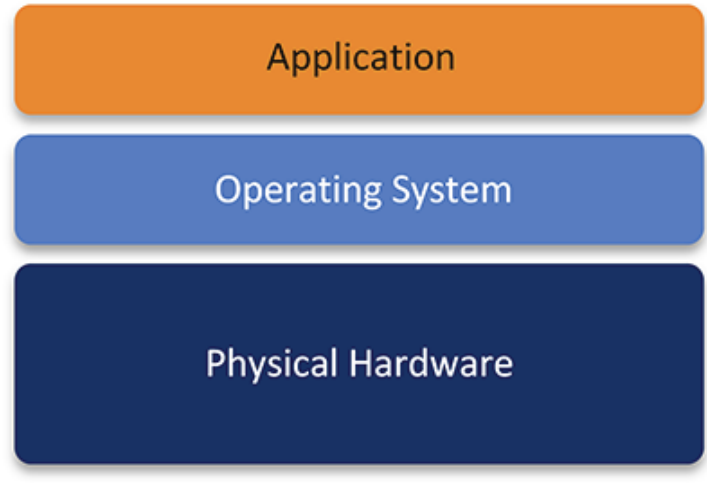

Traditional Architecture

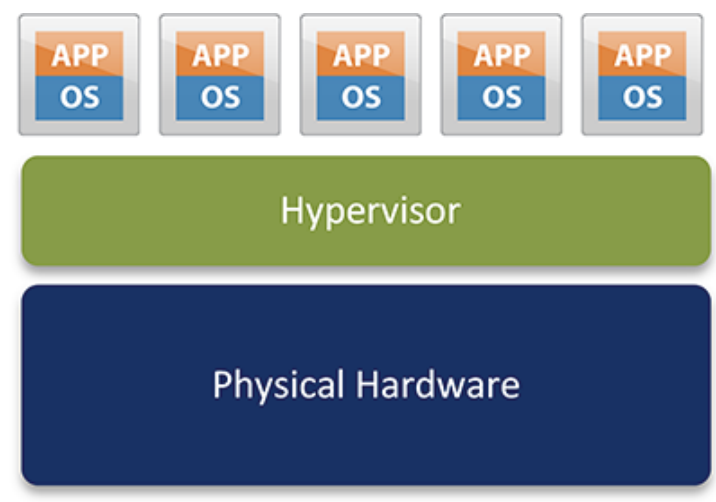

Virtual Architecture

Figure 2.8: Traditional and virtual architecture

Virtualization is often defined as system virtualization (CPU, memory, etc.). However, there are other types of virtualization as well. The most common types of virtualization are as follows 74 : 
- System virtualization: There are two varieties of system virtualization, namely server virtualization, and desktop virtualization.

Server virtualization is the most common form of virtualization. This type of virtualization adds a layer of abstraction (called hypervisor) between the physical server hardware and the software that runs on the server. This hides the characteristics of the physical server (CPU, memory, hard drive, etc.) from users, and converts the physical server into numerous independent virtual machines. Server virtualization results in efficient resource utilization, decreases the power consumption, and increases the flexibility. Amazon EC2 is an example of this type of virtualization.

Desktop virtualization is concerned with virtualizing the traditional desktop system, and moving the execution of the client's workload to the data center. The user has access to the remote system, which is hosted in the data center via different methods (e.g., thin client). It facilitates data backup and recovery, improves the system security, provides more flexibility in the desktop environment, and reduces the IT costs. Amazon WorkSpaces is an example of this type of virtualization.

- Network virtualization: This type of virtualization is about creating logical, virtual networks that are independent of the underlying network infrastructure. Also, it may divide a single network into multiple logical networks (VLAN). In essence, network virtualization abstracts physical networks into logical virtual networks that are decoupled from physical infrastructure. It solves many networking challenges by helping organizations to provision the network without having to touch the underlying infrastructure. VMware NSX is an example of this type of virtualization that enables the creation of entire networks in software and embeds them in the hypervisor layer.

- Storage virtualization: This type of virtualization is about grouping multiple network storage devices and logically presenting them as a single storage entity. This procedure abstracts the storage and hides its characteristics from the application and operating 
system. Also, it facilitates the storage allocation and enables the storage management in a centralized fashion. IBM SAN Volume Controller (SVC) is one of the known solutions for this type of virtualization.

- Application virtualization: This type of virtualization is about running an application on a remote server. The end user accesses the application over the network or by using a remote display protocol. Application virtualization is typically used to address incompatibility issues with the local operating system, or lack of sufficient resources to run the application on the user's machine. Microsoft Application Virtualization (formerly known as Microsoft Softgrid) is one example of application virtualization.

Today, there are numerous solutions for virtualization such as KVM, VMware ESXi [26], Microsoft Hyper-V [8], etc. Among these solutions, KVM is open-source and more suited for Unix-based systems. To build a virtual infrastructure for our study, we used OpenStack [18 with KVM, which is the recommended hypervisor for OpenStack.

\subsubsection{OpenStack}

OpenStack [18] is an open-source cloud computing platform founded by NASA and Rackspace. Today, it is being managed by OpenStack Foundation, and has many contributors from across the world. OpenStack can be deployed as an Infrastructure as a Service (IaaS) in the public cloud providers (e.g., AWS, Rackspace, Azure, etc.) or as a virtualization solution for companies of any size. OpenStack is Python-based, which allows it to be flexible and scalable. OpenStack consists of different components that are well-integrated and work together. The components of OpenStack communicate through RESTful APIs.

The following are the essential components of OpenStack:

1. Horizon: It provides a modular Web-based user interface (UI) for OpenStack services and users. Using its Web GUI, the majority of cloud operation such as launching, mi- 
grating, and deleting virtual machines can be done. Also, it enables the management of OpenStack networks, tenants, storage, etc.

2. Keystone: It enables central authentication and authorization for OpenStack users and services. It allows the cloud administrator to grant certain access to users or group them based on the level of required access to services and resources. Keystone can also integrate with external authentication protocols such LDAP or Radius.

3. Nova: Nova is the core of OpenStack, since it provides scalable, on-demand, and selfservice access to compute resources. Nova has different components (e.g., Nova-api, Nova-compute, Nova-scheduler, Nova-conductor, etc.). Besides, Nova interacts with other OpenStack services to fulfill user requests.

4. Neutron: It deals with networking tasks in OpenStack. Neutron (formerly called Quantum) creates and manages virtual network(s) between virtual machines, and routes the traffic between them. Neutron also can deliver firewall features, such as restricting ports and protocols for ingress or egress traffic. It allows users to create and manage their own network through Horizon, without knowing the complexity of OpenStack's multi-tenant network infrastructure.

5. Glance: It provides a repository and a catalog for operating systems images. Glance provides a service for users to upload and discover data assets that can be used with other services (e.g., images, metadata definitions). Glance service includes discovering, registering, and retrieving VM images. It can store image metadata in Swift or other storage repositories.

6. Swift: It is the object storage service of OpenStack. Swift is like a container that can store files and retrieve them later. Swift can be considered as cost-effective storage that provides a Dropbox-like service. It is well-suited for VM templates, ISO images, disk volume snapshot, backup, archive, and unstructured data. 
7. Cinder: It is the block storage service of OpenStack that provides persistent storage for the compute instances. Cinder enables block devices to offer expanded storage to VMs. It also allows manipulation of volumes, volume types, and snapshots through API. Cinder provides an Amazon EBS-like service and is ideal for performancesensitive applications, such as database-driven applications, etc.

Figure 2.9 presents a conceptual view of OpenStack's key components, and the relationships between them.

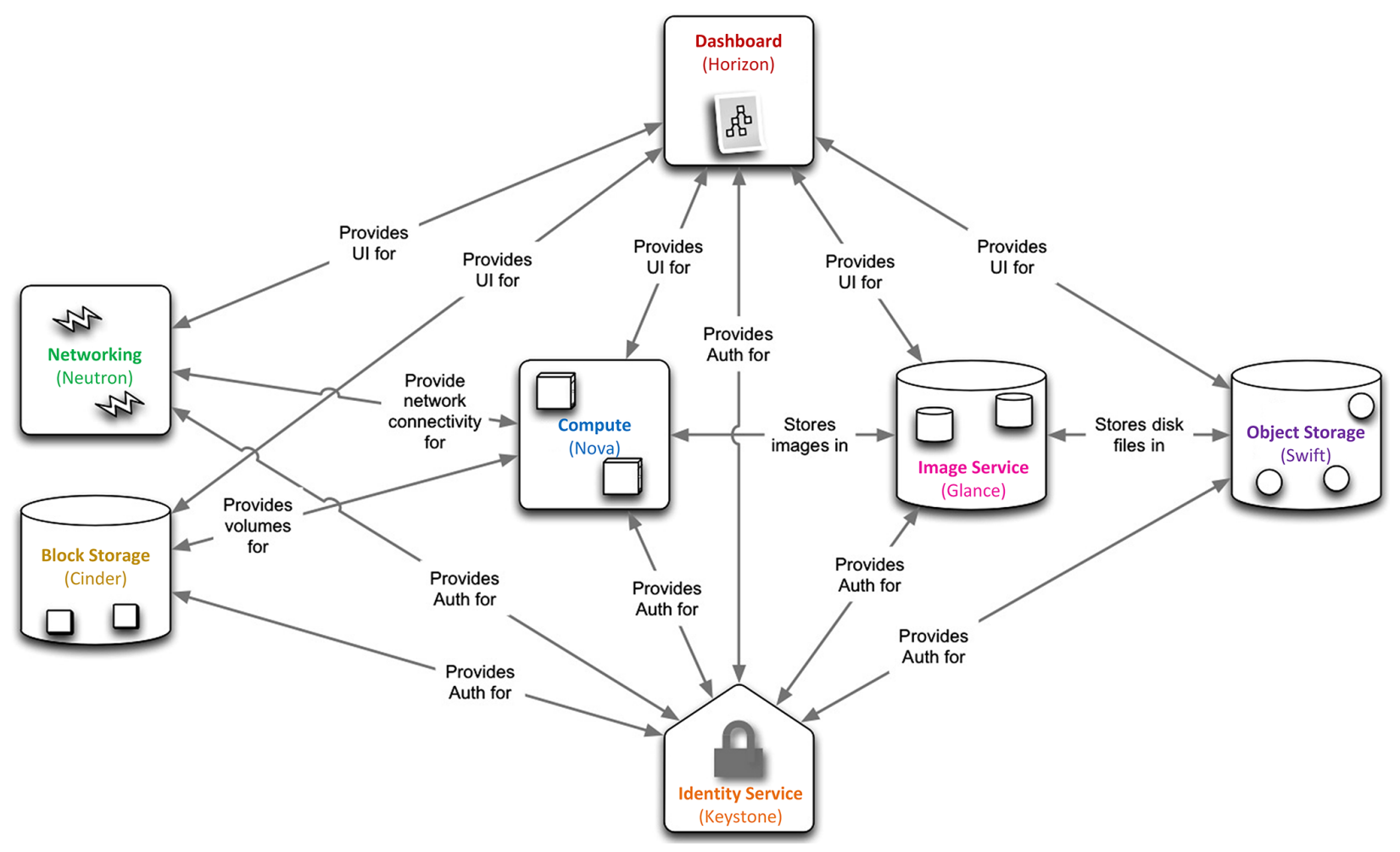

Figure 2.9: Conceptual view of OpenStack's main components 18

The impacts of virtualization on the performance of applications, systems, and networks have been studied in prior work such as [67] [94] [95]. Wang et al. 95] studied the impact of virtualization on file synchronization in Dropbox. The authors discovered a performance bottleneck that increases synchronization delay among multiple users in virtual machines. They proposed a solution to reduce the synchronization delay for virtual machines. 


\subsection{Hyper-Threading}

Hyper-Threading is Intel's implementation of Simultaneous Multi-Threading (SMT) [91] 92 to improve the parallelization of computations on x86 microprocessors. SMT speeds up the execution of jobs by fetching and executing multiple code streams, and increases the utilization of the execution resources in the processor. Hyper-Threading was first introduced in 2002 on Xeon processors of Pentium 4 desktop computers. Then, Intel included this technology in Atom, Itanium, and Core "i' series CPUs ${ }^{4}$.

With Hyper-Threading, each physical processor core appears as two virtual (logical) cores for the operating system. Figure 2.10 shows a schematic view of Hyper-Threading.
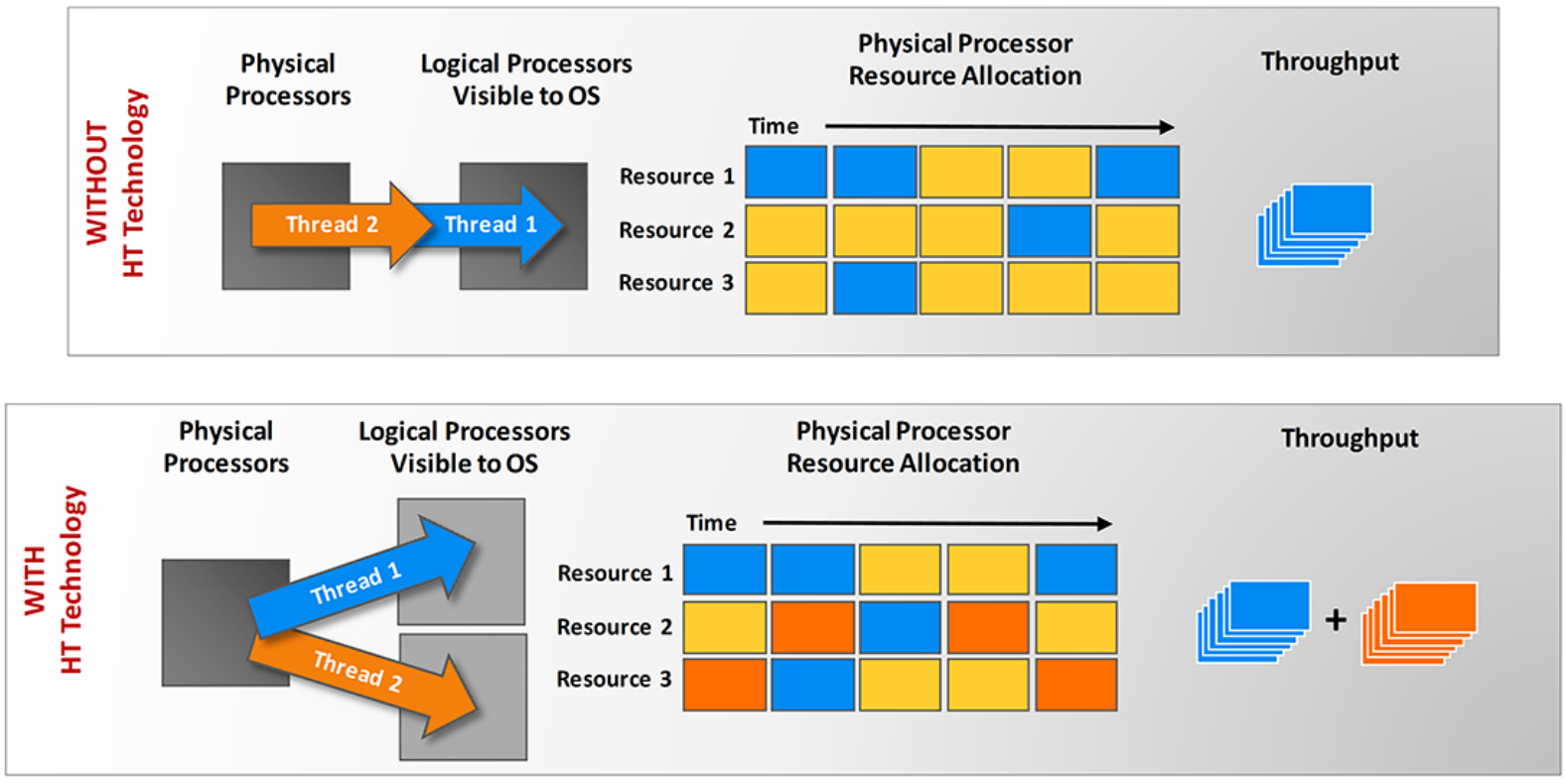

Figure 2.10: Hyper-Threading technology concept $\mid 5$

Each logical processor maintains a complete view of the architecture state, including registers. The registers comprise the control registers, the general-purpose registers, the Advanced Programmable Interrupt Controller (APIC) registers, and some machine state registers. From the software point of view, when the architecture state is duplicated, one

\footnotetext{
${ }^{4}$ https://en.wikipedia.org/wiki/Hyper-threading
} 
processor appears as two processors. Thus, it doubles the CPU's throughput [68]. Each (logical) processor can consume resources that are not used by the other logical core. Hence, the other tasks can run and progress simultaneously. This increases the overall utilization of execution resources, which results in improved software performance in I/O-intensive tasks and multi-threading environments. Logical cores share all the resources on the physical processor, such as control logic, buses, execution units, caches, and branch predictors.

Hyper-Threading is suitable for operations and applications where multiple tasks can be effectively scheduled. Thus, there's no idle time on the processor. For instance, video editing and 3D rendering are good examples that benefit from Hyper-Threading. On the other hand, operations where tasks have to be done in serial, or where one operation has to take place before another can begin, usually do not benefit from Hyper-Threading 5 .

There have been numerous studies focusing on the impact of Hyper-Threading on the performance of different applications or platforms.

Saini et al. [79] studied the impact of HT on production applications. In particular, they examined the impact of HT on four applications of interest to NASA, namely Cart3D, NCC, OVERFLOW, and USM3D. The result of their study shows that the performance of Cart3D, NCC, and USM3D is better when HT is enabled. However, OVERFLOW does not show any performance improvement with HT.

In another study [41], the authors focused on evaluating the impact of HT on HighPerformance Computing (HPC). They used High-Performance LINPACK (HPL) software. HPL is used to generate and solve dense linear systems of equations on distributed-memory computers. They used two different math libraries with HPL, namely Goto and Atlas, to evaluate the impact of HT on cluster configuration. They ran the HPL on a cluster of 8 servers. For each library, they ran HPL with and without HT. Their findings show that enabling Hyper-Threading may improve or worsen the performance, depending on characteristics of the software, such as communications or memory access patterns, and cache size.

\footnotetext{
5 http://lifehacker.com/how-hyper-threading-really-works-and-when-its-actuall-1394216262
} 
The authors mentioned that applications using fine-tuned mathematical libraries have less chance to benefit from Hyper-Threading.

\subsection{Related Work in Performance of OpenFlow Controllers}

There have been numerous studies focusing on the performance of OpenFlow controllers in the past couple of years. The majority of them conducted a benchmarking study on some centralized controllers such as Beacon [44], Floodlight [3], Maestro [38], NOX [50], POX [20], Ryu [22], and so on. These studies were designed to identify the baseline performance of controllers, and determine which controller outperforms the others in certain tests. On the other hand, some other studies prototyped and proposed new controllers with advantages over other controllers regarding throughput and latency. In this category, typically the authors conducted an experimental evaluation on the proposed controller as well as some other controllers to validate their claim. However, mostly the advantages were transitory. This is mainly because the other controllers improved in performance in subsequent versions, and the performance gap disappeared.

In a highly cited study, Tootoonchian et al. [90] evaluated the performance of several OpenFlow controllers. The authors presented NOX-MT, a multi-threaded version of NOX controller. NOX-MT employs well-known optimization methods (e.g., I/O batching) to boost the baseline performance. Their experimental results show that NOX-MT has 33 times better overall performance than NOX. Also, they designed a benchmarking tool called "Cbench" that enables researchers to gauge the throughput and latency of OpenFlow controllers by emulating OpenFlow switches. As part of their work, they evaluated the performance of Beacon, Maestro, NOX, and NOX-MT. Their results showed that the performance of all examined controllers was significantly better than what the literature was assuming. In their study, NOX-MT outperformed the other controllers in almost every test.

Fernandez [48] focused on the operation modes for OpenFlow controllers. The author 
evaluated the performance of Floodlight, NOX, POX, and Trema [24] in both reactive and proactive modes. The conducted study included two different scenarios. In the first scenario, he used a real network with Netgear switches, and in the second scenario, he used a virtualized network built with Mininet. The results indicate that the performance of all controllers is better in proactive mode. In proactive mode, the controller receives fewer request messages from the switch, since the paths are configured in advance. Finally, the author proposed a new mode of operation named hybrid. In the suggested model, the controller behaves like reactive mode and installs flows on demand. However, it has the intelligence to understand the traffic behavior, and defines the route in advance, acting like proactive mode. The author stated that this approach leads to performance improvement.

Shah et al. 81 studied the architecture of OpenFlow controllers. Their goals were to identify the key performance bottlenecks, and suggest architectural elements to improve the performance of existing OpenFlow controllers. They benchmarked four OpenFlow controllers including Beacon, Floodlight, Maestro, and NOX with different performance metrics in a customized testbed. Their results indicate that the Beacon controller outperformed the other controllers in the throughput and latency tests. The authors noticed that these controllers have different architectural features. They outlined the cornerstones of these controllers and categorized them as either Switch Partitioning, Multicore Support, Packet Batching, and Task Batching. Lastly, they proposed a new controller that outperformed Beacon by 11.5\%, $11.6 \%$, and $2.7 \%$ in throughput, latency, and thread scalability. They indicated that the use of static packet batching and switch partitioning enables higher throughput, while adaptive packet batching and task batching results in better latency.

Shalimov et al. 83 conducted an experimental evaluation of some of the well-known OpenFlow controllers, namely Beacon, Floodlight, Maestro, MUL, NOX, POX, and Ryu. The aspects of their work were reliability, performance, security, and scalability. The reliability analysis demonstrated that all of the mentioned controllers can deal with the average 
workload except Maestro and MUL. They also implemented and presented hcprobe, a framework to benchmark OpenFlow controllers.

In 2015, Khattak et al. [60] conducted a preliminary study of the Floodlight and OpenDaylight controllers using Cbench. They evaluated the performance of these controllers in throughput and latency tests. They chose Floodlight because it is a mature SDN controller. The authors noticed that OpenDaylight has unexpectedly poor responsiveness in throughput and latency tests compared to Floodlight. They mentioned that OpenDaylight failed to produce any result in many of their tests, and this failure increased when scaling up the number of switches. They speculated that this could be due to memory leakage of the OpenDaylight controller. They also proposed modifications in Cbench to support data center traffic models.

In recent work, Salman et al. [80] studied the performance of well-known centralized (Beacon, Floodlight, IRIS, Libfluid_MSG, Libfluid_RAW, Maestro, MUL, NOX, POX, and Ryu) and distributed (ONOS and OpenDaylight) OpenFlow controllers. The authors used WCbench [27], which is a Python wrapper around Cbench, for their evaluation. The results of their experiments indicate that controllers written in $\mathrm{C}$ have the highest throughput (e.g., MUL and Libfluid_MSG). Following them, Java-based controllers such as Beacon, IRIS, and Maestro demonstrated higher throughput compared to other controllers. On the other hand, in the latency test, Maestro showed the best latency among all examined controllers, since it used adaptive batching. They mentioned that controllers coded in $\mathrm{C}$ and Java could use many threads, whereas Python-based controllers do not show better performance when increasing the number of threads.

In another performance study, Zhao et al. [99] carried out an evaluation study on the performance of Beacon, Floodlight, NOX, POX, and Ryu. They conducted their experiments in a controlled testbed, with optimized configuration for each controller. Their results indicate that Beacon outperformed other controllers in throughput and latency tests. Floodlight was 
about average, and NOX suffered from scalability issues. Ryu and POX demonstrated that they could handle $100 \mathrm{~K}$ packets per second.

In 2014, Shin et al. 85 introduced Rosemary as a robust and high-performance controller. The authors performed some throughput tests on Rosemary and other controllers (Beacon, Floodlight, and NOX) to evaluate and compare their performance. They implemented Rosemary in two modes, namely U-App (User-Application) and K-App (Kernel-Application). U-App Rosemary showed a fixed throughput of 5.5M (resp/s) regardless of the number of switches. K-App Rosemary demonstrated better performance, and it showed a throughput of $6 \mathrm{M}(\mathrm{resp} / \mathrm{s})$ with a single switch, and a throughput of $8 \mathrm{M}(\mathrm{resp} / \mathrm{s})$ for 4 switches and more. U-App and K-App are limited to 2 and 4 threads, respectively.

Ivashchenko et al. [56] proposed an OpenFlow controller named In-Kernel, which works as a module inside the Linux kernel. They compared the throughput of In-Kernel with other controllers such as Beacon, Floodlight, Maestro, MUL, NOX, POX and Ryu. They ran controllers with 1-12 CPU threads, and used Cbench to evaluate their throughput. With 12 threads, In-Kernel showed a throughput of 30M (resp/s) and outperformed other controllers. Following In-Kernel, Beacon had better performance compared to other controllers, and showed a throughput of $7 \mathrm{M}(\mathrm{resp} / \mathrm{s})$.

In 2013, Erickson from Stanford University introduced the Beacon controller [44]. His work outlines the design and architecture of Beacon. The author also compared the performance of Beacon with Floodlight, Maestro, NOX, POX and Ryu. He tested controllers with Cbench with single and multiple threads. In both experiments, Beacon showed higher throughput compared to other controllers $(1.4 \mathrm{M}$ resp/s with a single thread, $10 \mathrm{M}$ resp/s with 12 threads).

Cai from Rice University focused on Maestro controller in his PhD dissertation [38. In his work, he investigated software design choices for the OpenFlow controller. In the design of Maestro, he used static partitioning and static batching techniques that were used 
previously in NOX and Beacon controllers. He examined four different switch partitioning techniques in his design, namely shared-queue, static, dynamic, and round robin. As part of his work, he evaluated the performance of Beacon, Maestro (with each of these techniques), and NOX. His results indicated that Maestro with static batching technique has a higher throughput compared to other controllers in his study.

\subsection{Summary}

In this chapter, we described the foundation and architecture of Software-Defined Networking, and explained the main components of OpenFlow. We described two OpenFlow controllers that we will evaluate to assess their performance. Also, we described virtualization and Hyper-Threading technologies, since they are part of our study. Lastly, we reviewed the related work on the performance evaluation of OpenFlow controllers.

In the next chapter, we will discuss our performance testing methodology and metrics. Also, we will describe our testbed, experimental structure, and the tool used for evaluation of the controllers. 


\section{Chapter 3}

\section{Methodology and Experiment Setup}

\subsection{Overview}

In this chapter, we discuss the methodology and metrics of our experimental evaluation. To be more specific, we explain throughput, latency, and thread scalability as our primary performance metrics. Then we describe the physical and virtual testbeds for our experimental evaluation. We also discuss our experimental structure, phases, and parameters. Lastly, we explain Cbench, which is the benchmarking tool that we used to evaluate controllers.

\subsection{Methodology and Metrics}

We employed a similar benchmarking methodology to prior studies [80] [83] [85] [90] to ensure the accuracy of our results. Our methodology studies performance and thread scalability. The performance of an SDN/OpenFlow controller is identified by two characteristics: throughput and latency [83]. In the multi-threaded controllers, such as ONOS and OpenDaylight, the scalability of the controller is another essential dimension to consider.

In this thesis, we quantify the performance of ONOS [11 35] and OpenDaylight [14 66 with Cbench [1]. Our experimental evaluation examines the performance of single-node ONOS and OpenDaylight controllers. Understanding the performance of a single-node is useful since it provides a baseline to compare ONOS and OpenDaylight with each other, and to other OpenFlow controllers.

We conduct our experiments on stand-alone ONOS and OpenDaylight in three phases. First, we benchmark the throughput, latency, and scalability of the controllers on the physical server (bare-metal) with Hyper-Threading enabled. Second, we perform the same benchmark 
tests on ONOS and OpenDaylight on the physical server without Hyper-Threading. Finally, in the last phase, we benchmark ONOS and OpenDaylight when running in the virtual host.

\subsubsection{Throughput, Latency, and Thread Scalability}

As mentioned in Chapter 2, in software-defined networks, when a new packet flow arrives, the switch checks its flow table(s) to find a match based on the packet's header fields. If the switch finds a match, it applies the corresponding action. If not, it sends the packet (or part of it, depending on the configuration) to the controller through a packet_in message. The controller then replies back with the final decision (action) for that packet, which can be a packet_out message with instructions to forward the packet or drop it entirely. Also, it is possible that the controller installs a flow rule for that packet in the flow table of the switch with a flow_mod message, in case another packet from the same flow arrives [76].

The throughput indicates the number of flow setup requests that the controller can handle per unit of time. The throughput of the control plane is a major factor in determining the overall number of controllers required to handle traffic load on a network.

On the other hand, latency is the time required for the controller to process a packet_in message and send back a packet_out message. The latency between the controller and OpenFlow switch is of vital importance, since it impacts the performance of the entire network. Also, this metric helps to understand the controller response time under an event like network path failure.

In multi-threaded controllers, thread scalability is an issue that impacts the performance of the controller. In single-threaded controllers, the correlation between throughput and latency is quite straightforward. In such controllers, throughput is inversely proportional to latency. However, in multi-threaded controllers, such as ONOS and OpenDaylight, throughput exceeds the inverse of the latency, since packets can be processed in parallel. Thread scalability indicates a controller's efficiency in utilizing available threads to increase the throughput and reduce the flow setup latency. 


\subsection{Testbed Setup}

The testbed is constructed in the TELUS Labs in Toronto. The testbed consists of three Cisco UCS C240 M4SX rackmounted servers. Each server is equipped with two Intel Xeon E5-2670 CPUs (24 cores @ 2.30 GHz), 256 GB RAM, 4 TB hard drive, and two NICs 1 Gbps and 10 Gbps). All servers run Ubuntu 14.04.4 LTS x64 operating system. The servers connect through a Cisco UCS 6248 Fabric Interconnect that operates in Ethernet Switching mode. The Fabric Interconnect is a layer 2 switch with advanced features, such as high port density, high bandwidth, low port-to-port latency, etc. The Cisco UCS C240 M4 servers are enterprise-class servers designed to deliver high performance, and are ideal for a broad spectrum of storage and I/O-intensive infrastructure workloads.

Each server has 24 cores (48 threads with HT), and 2 CPU sockets. With HyperThreading, each processor socket has 24 threads. Threads $0-11$ and 24-35 reside on socket 1, and threads 12-23 and 36-47 reside on socket 2. Without Hyper-Threading, each processor has 12 threads. Threads $0-11$ reside on socket 1 , and threads $12-23$ reside on socket 2 . Tables 3.1 and 3.2 present the number of processor sockets, CPUs, cores, and threads per server with and without Hyper-Threading.

Table 3.1: Total CPU cores and threads per server (HT enabled)

\begin{tabular}{|c|c|c|c|c|}
\hline Sockets & Cores per Socket & Threads per Core & Total Cores & Total Threads \\
\hline 2 & 12 & 2 & 24 & 48 \\
\hline
\end{tabular}

Table 3.2: Total CPU cores and threads per server (HT disabled)

\begin{tabular}{|c|c|c|c|c|}
\hline Sockets & Cores per Socket & Threads per Core & Total Cores & Total Threads \\
\hline 2 & 12 & 1 & 24 & 24 \\
\hline
\end{tabular}

\footnotetext{
${ }^{1}$ The 10 Gbps NIC is used for network traffic, and 1 Gbps NIC is used for out-of-band management. We separate the network traffic from management traffic in our testbed.
} 
Figure 3.1 portrays our physical testbed structure. As shown below, the experiments on the physical host use two servers. One server is used to run Cbench, and the second server is used to run the controllers (one controller at a time).

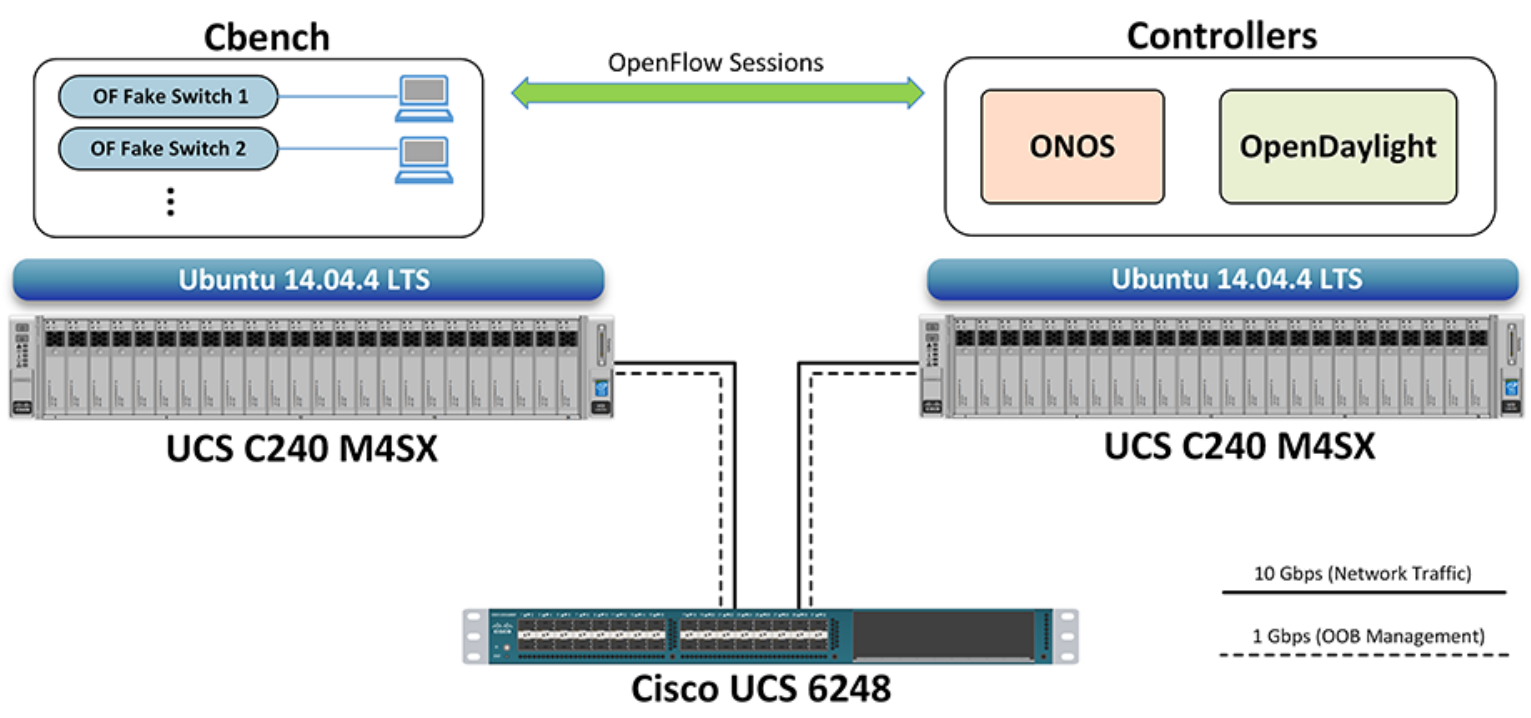

Figure 3.1: Physical testbed structure

We run the experiments with two servers instead of a single shared server for the following reasons. First, the throughput of operating system internal traffic can be erratic [83]. Second, with two servers, we assure that Cbench traffic generation does not affect the controller's operation and overall performance. Lastly, with two servers, we circulate the OpenFlow messages in the network, which reflects OpenFlow controller-switch communication in a real network.

Figure 3.2 illustrates our virtual testbed structure. We deploy OpenStack Kilo version on three servers. One server (labeled as controller_node) has the role of OpenStack controller, and the other two servers are compute nodes. The controller node runs OpenStack's services such as scheduler, Web interface, database server, message queue, etc. The compute nodes run the hypervisor, nova-compute service, and neutron agents. The VMs are run on compute nodes. 


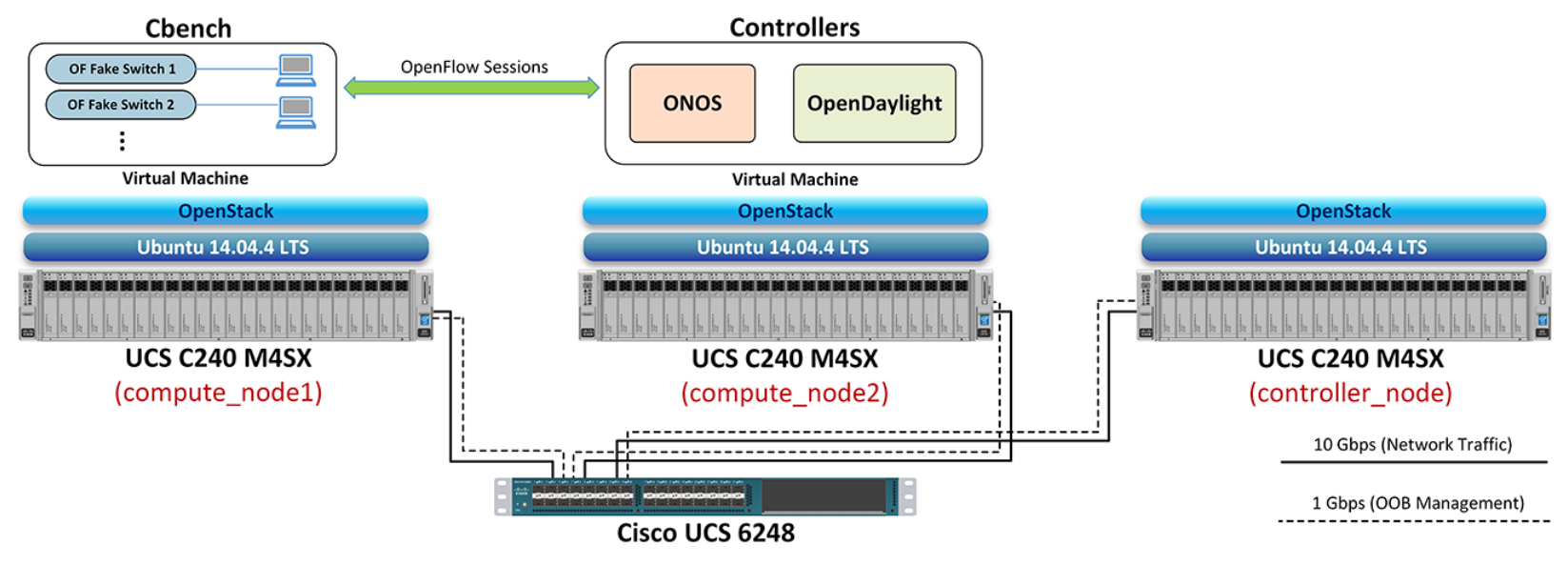

Figure 3.2: Virtual testbed structure

Table 3.3 summarizes the CPU and memory of the controller and Cbench VMs.

Table 3.3: Controller and Cbench VM specifications

\begin{tabular}{|c|c|c|c|c|}
\hline VM & Host & vCPU & RAM & vNIC \\
\hline Cbench & compute_node1 & 8 & 16 GB & 10 Gbps \\
\hline Controllers & compute_node2 & 20 & $20 \mathrm{~GB}$ & $10 \mathrm{Gbps}$ \\
\hline
\end{tabular}

As shown in Figure 3.2, we run controllers and Cbench in separate VMs on separate compute nodes for consistency with settings on the physical host. We set the overcommit ratio to 1 in the nova configuration, so that 1 vCPU and 1 GB RAM of each VM correspond to 1 pCPU and 1 GB RAM on the bare-metal host. For the controller VM, we use 16 vCPUs and 16 GB of memory for running the controller. The remaining 4 vCPUs and 4 GB memory are used to run the operating system.

In the virtual environment, VM communications occur through virtual switches along with the physical switches. There are numerous virtual switches such as Open vSwitch (OVS), VMware Virtual Switch, Cisco Nexus 1000v, etc. OVS $[13]$ is an open-source switch designed for Linux-based hypervisors (e.g., KVM and XEN), and is typically used with OpenStack. In the OpenStack environment, there is an OVS per server. The vNIC(s) of the 
VMs running on each server are connected to the OVS of that server, and the OVS is linked to the server's physical NIC. The OVS supports VLAN tagging of the physical switch, which enables layer 2 traffic isolation.

Figure 3.3 depicts the physical and virtual network that connect the controller and Cbench VMs.

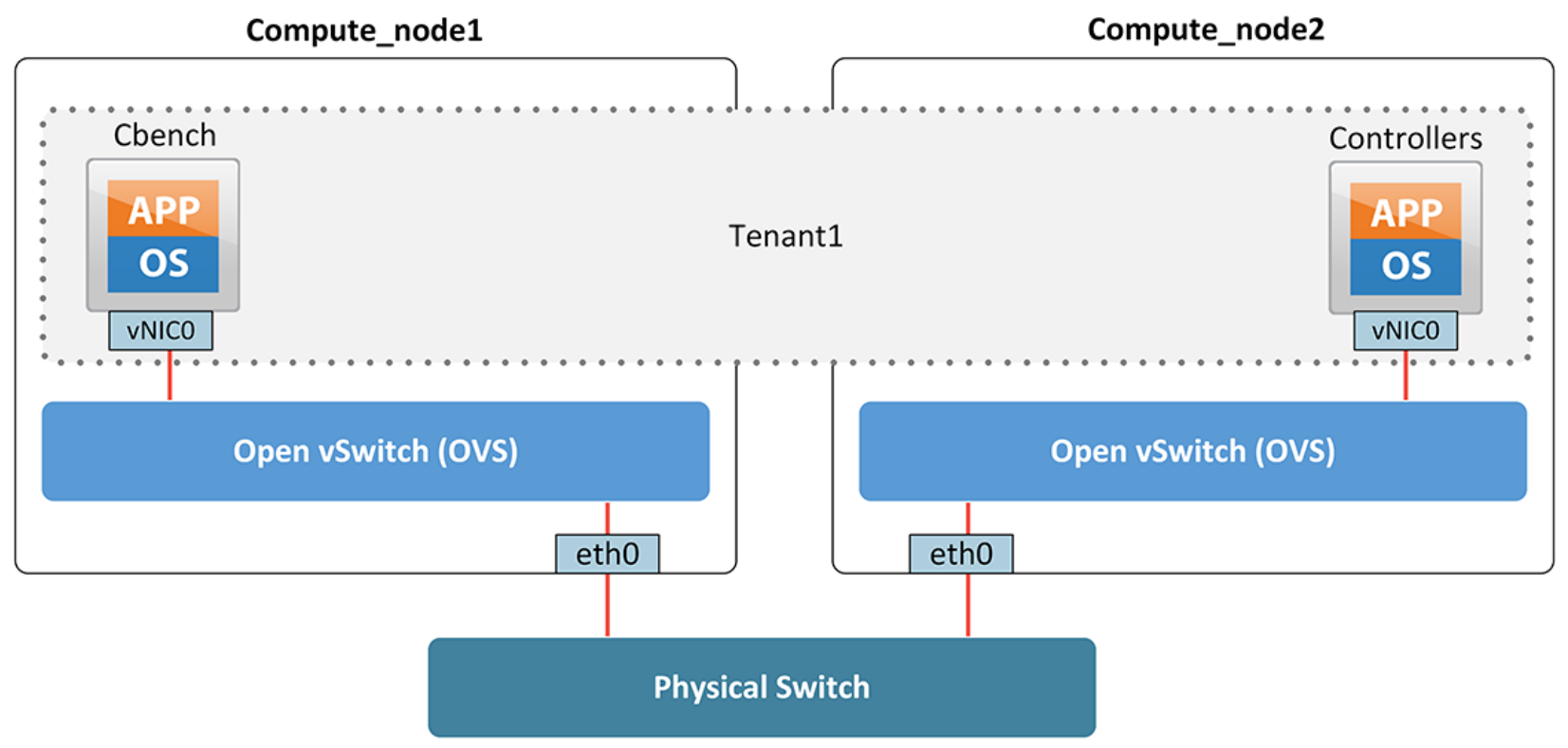

Figure 3.3: Physical/Virtual network of the controller and Cbench VMs

For simplicity, and to avoid Inter-VLAN routing, we run the Cbench and the controllers within the same OpenStack tenant so they are in the same layer 2 broadcast domain. That being said, we have a flat network with a default VLAN.

We performed some network throughput tests between the Cbench server and the controllers to identify the end-to-end link capacity, and ensure there is no bottleneck to impact the performance of controllers. To this end, we used iPerf [7] with its GUI (JPerf) to measure the maximum achievable throughput between VMs. Each server is connected to the Fabric Interconnect with a 10 Gbps link. We measured the throughput with 1-4 TCP streams. As shown in Figure 3.4, the aggregate network throughput is 8 Gbps or more for communication between compute nodes. 


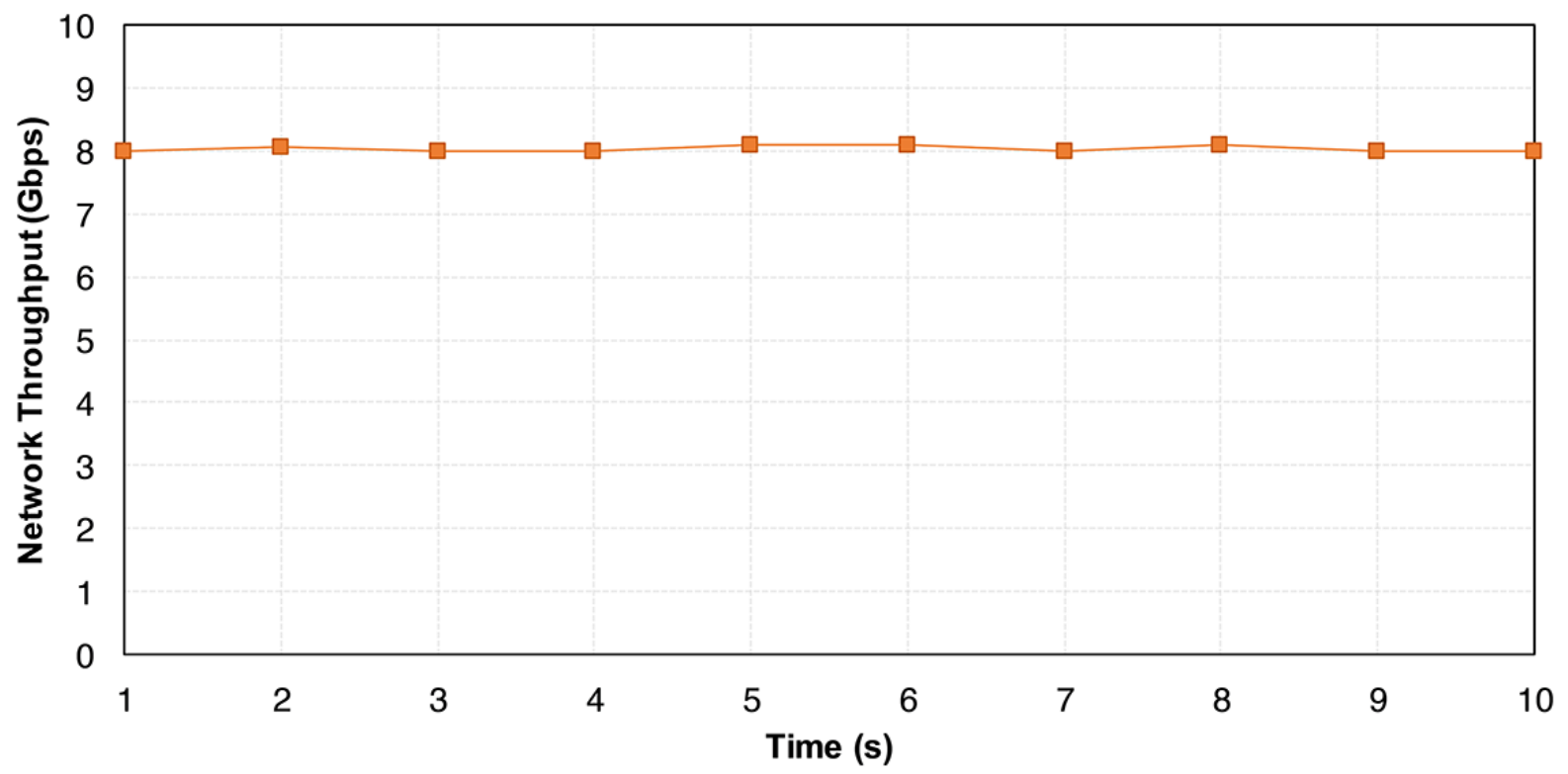

(a) Network throughput test with 1 TCP stream

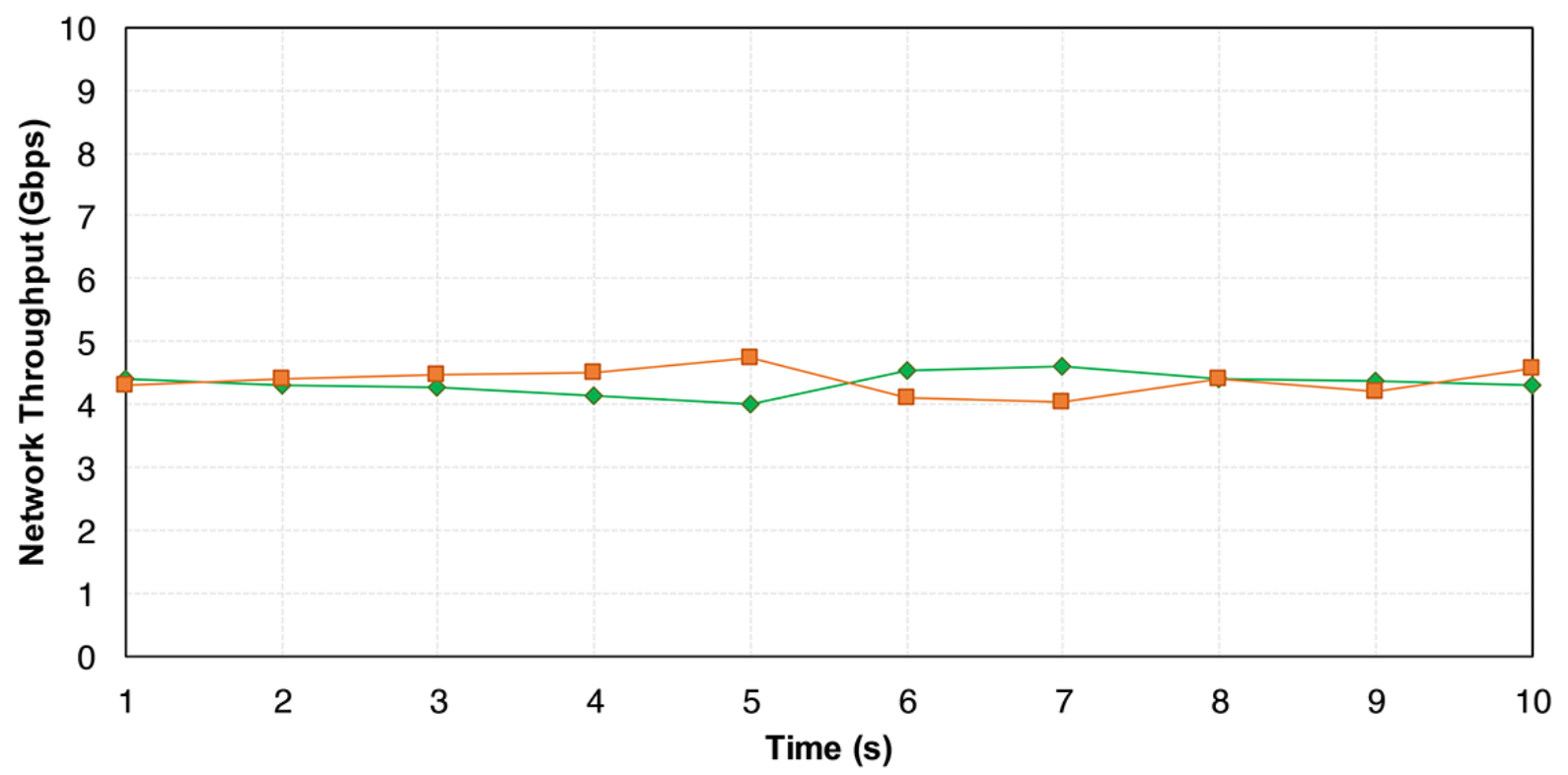

(b) Network throughput test with 2 parallel TCP streams 


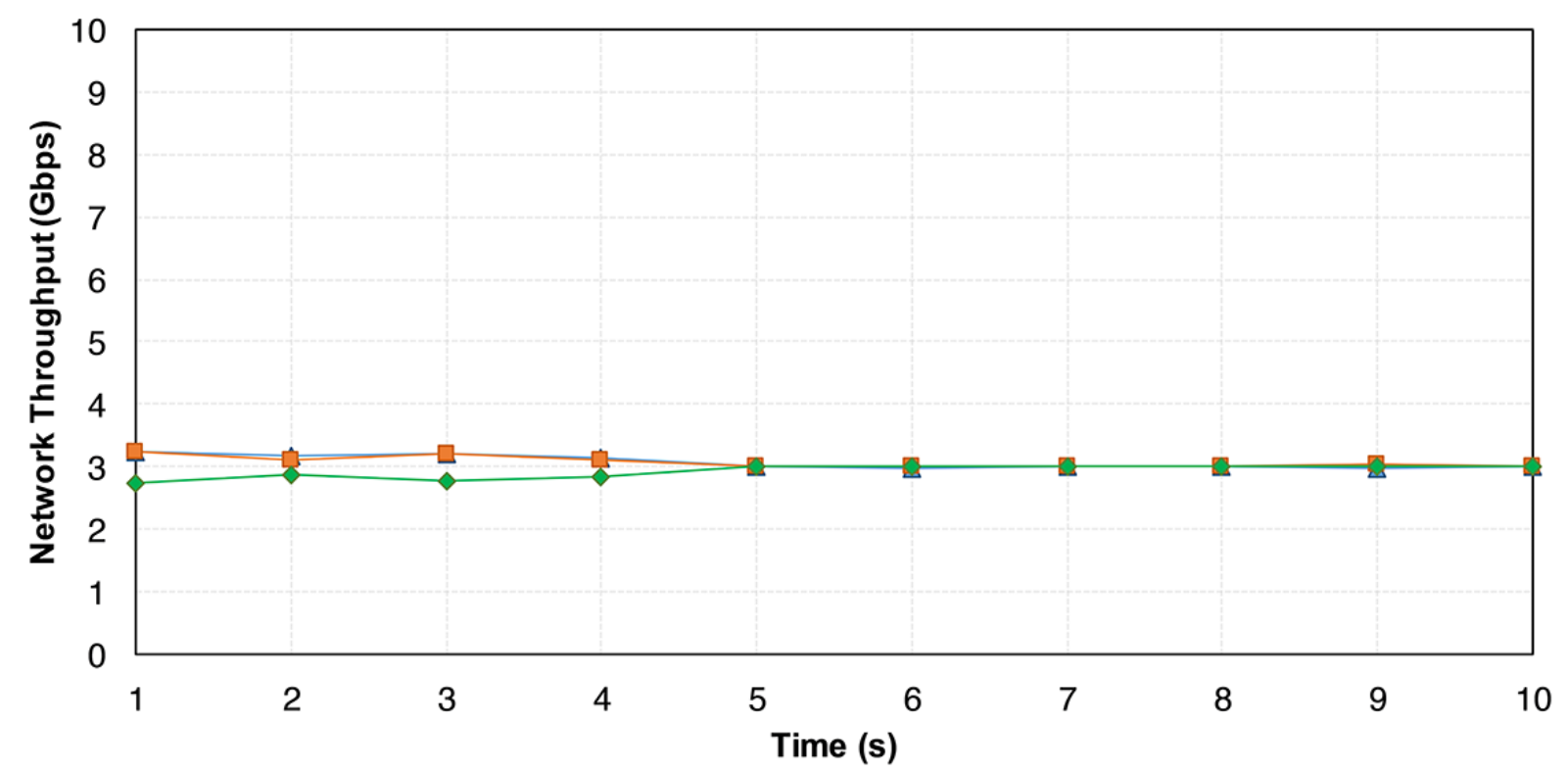

(c) Network throughput test with 3 parallel TCP streams

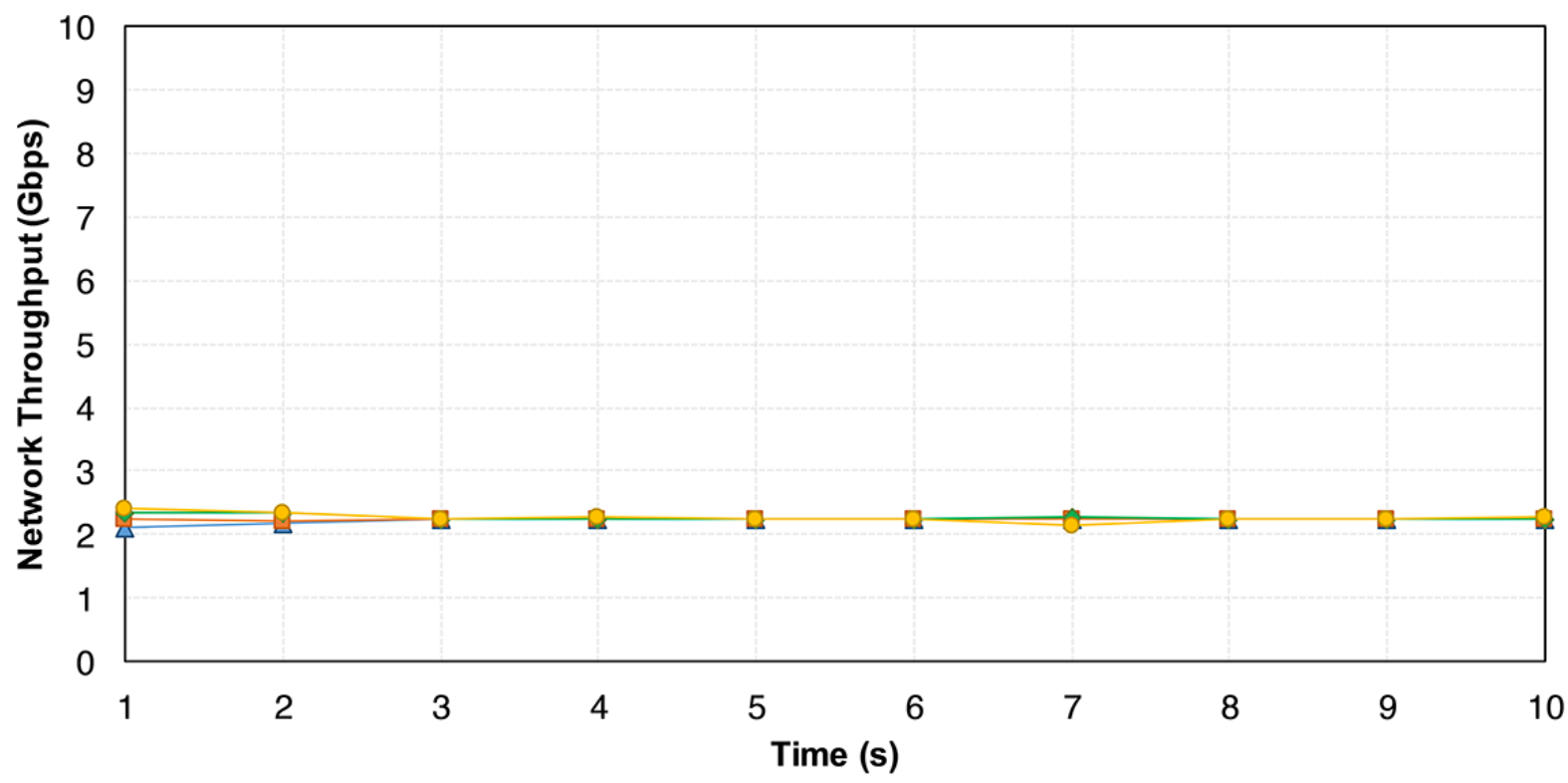

(d) Network throughput test with 4 parallel TCP streams

Figure 3.4: TCP throughput test between Cbench and controller VM 


\subsection{Experimental Setup}

We run all experiments in three phases. In first two phases, we perform the experiments on the physical host, and in the third phase, we perform the tests on the virtual host. In the first phase, we conduct the experiments with Hyper-Threading, since it is enabled by default. In the second phase, we disable Hyper-Threading and perform the experiments. Lastly, we construct a virtual testbed and perform the tests on the virtual host. As shown in Figure 3.5, Hyper-Threading is controlled at the hardware BIOS level.

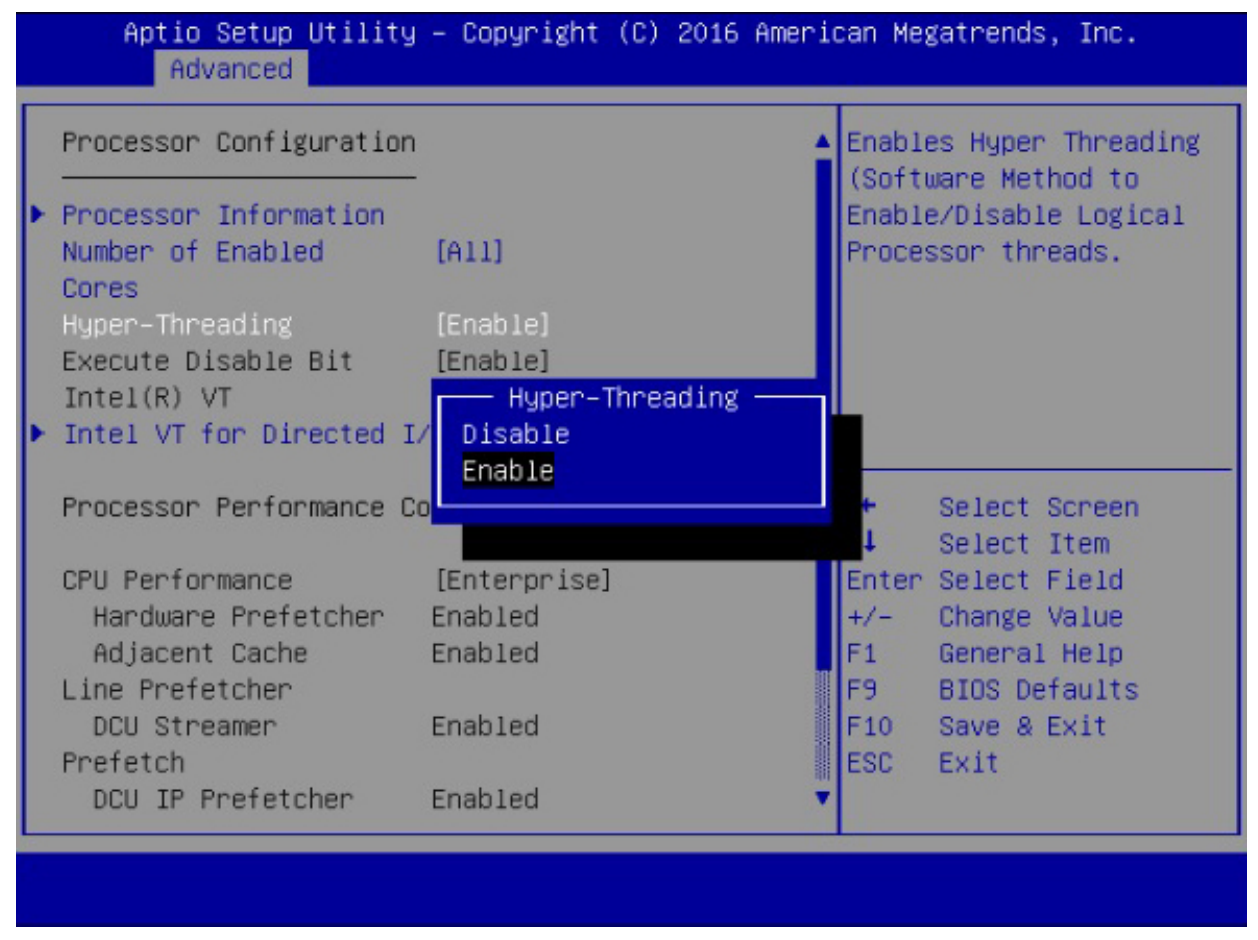

Figure 3.5: Hyper-Threading option in the BIOS menu

To precisely measure the performance of each controller using CPU threads, we prohibit access to those threads by the operating system and other applications. To this end, we use the isolcpus command to isolate the desired number of threads before running the experiment. Then, to run the controller, we use the taskset command to bind the isolated threads to the controller. Also, after each round of tests, we shut down and restart the controller to have a clear cache for the next round. 
Moreover, to ensure that processor affinity (thread pinning) works well with taskset, before running the experiments we examine taskset with the Linux stress tool. It is worth noting that we use ntop (monitoring tool) during all experiments to make sure that only specified CPU threads are being used, and load sharing is fair.

\subsubsection{Experimental Factors}

We assess the throughput and latency of controller based on the following parameters:

- Number of switches: The number of switches connected to the controller (1-512)

- Number of hosts: The number of hosts (MAC addresses) per switch $\left(10^{0}-10^{6}\right)$

- Number of threads: The number of threads assigned to the controller (1-16)

\subsubsection{Structure of Experiments}

For each test, we conduct 350 experiments. We perform our experiments in five groups (replications), and each group consists of 70 experiments with the duration of 5 seconds each. We consider the first 10 experiments of each group for the controller's warm-up and discard their results. Overall, we obtain 300 outputs for each test. Table 3.4 summarizes the number of experiments for each test.

Table 3.4: Rounds of tests per experiment

\begin{tabular}{|c|c|c|c|}
\hline Groups & Tests per Group & Discarded Tests per Group & Total Tests \\
\hline 5 & 70 & 10 & 300 \\
\hline
\end{tabular}

\subsubsection{Output of Experiments}

For each experiment, we obtain five average values and five standard deviations (Cbench calculates and prints these values at the end of each test). We used Matlab code and a function provided in [2] (included in Appendix A to calculate the overall average and standard 
deviation. The Matlab function calculates the combined standard deviation via ANOVA (Analysis of Variance). It calculates and returns the overall number of observations, the overall mean, and the overall standard deviation based on the number of observations, mean value, and standard deviation for each group. The overall standard deviation is calculated with the following steps:

Step 1) Square the standard deviation of each group to get the variance, $V(i)$. Multiply each variance by $n(i)-1$, the so-called "degrees of freedom" for each group to obtain the "Error Sum of Squares" of each group, $E S S G(i)$. The number of observations in group $i$ is $n(i)$.

$$
\operatorname{ESSG}(i)=V(i) \cdot(n(i)-1)
$$

Step 2) Sum the Error Sum of Squares of each Group (ESSG) to obtain the overall Error Sum of Squares (ESS).

$$
E S S=E S S G(1)+E S S G(2)+\ldots+E S S G(G)
$$

Step 3) Compute the deviation $\bar{Y}(i)$ of each group mean from overall grand mean (GM). Square each, and multiply by its $n(i)$.

$$
G S S(i)=(\bar{Y}(i)-G M)^{2} \cdot n(i)
$$

Step 4) Sum the obtained GSS of groups to get the total (overall) group sum of squares.

$$
T G S S=G S S(1)+G S S(2)+\ldots+G S S(G)
$$

Step 5) Add the overall Error Sum of Squares ESS from step (2) to the overall Group Sum of Squares TGSS from step (4) to get the "Total Sum of Squares". Then divide it by the "degrees of freedom" to obtain the grand variance. Degree of freedom is $N-1$, where $N$ is the total number of observations.

$$
G V=(E S S+T G S S) /(N-1)
$$


Then, take the square root of GV to get the overall standard deviation:

$$
\sqrt{G V}
$$

\subsection{Cbench}

Cbench (controller benchmarker) is the current standard tool for evaluating the performance of OpenFlow controllers [1] [90]. It emulates a configurable number of OpenFlow switches and hosts to measure different performance aspects of the controller. Cbench identifies controller's minimum, average, and maximum response time.

Cbench has two modes of testing, namely throughput and latency. In throughput mode, each OpenFlow switch constantly sends packet_in messages, and counts the corresponding packet_out messages. In latency mode, each OpenFlow switch sends a packet_in message to the controller, and waits for the matching packet_out message. Cbench calculates the average flow setup latency based on the total number of responses received during the test period. In a nutshell, Cbench measures the rate and the delay of packet_out messages to calculate the throughput and latency.

\subsection{Summary}

In this chapter, we discussed the details of testbed setup (including physical and virtual) and explained the methodology of our experimental evaluation. We also described the structure of experiments and reviewed Cbench, the benchmarking tool used in this study.

In the next chapter, we present the results of our experimental evaluation of throughput, latency, and thread scalability for ONOS and OpenDaylight controllers. 


\section{Chapter 4}

\section{Baseline Experimental Results}

In this chapter, we present the results of our experimental evaluation based on the performance metrics discussed in Chapter 3, namely throughput, latency, and thread scalability. Section 4.1 provides an overview of the tests. Section 4.2 discusses the results of throughput tests on ONOS and OpenDaylight. Section 4.3 presents the results of latency tests on the controllers. Lastly, we discuss the thread scalability tests in Section 4.4. Where possible, we compare the results of each test with previous studies to better understand the performance differences (if any).

\subsection{Overview}

As mentioned in Chapter 3, we conducted our experiments on stand-alone ONOS and OpenDaylight in three phases, with the objective to create a performance baseline and investigate which controller provides the highest throughput and lowest latency. First, we examined the throughput, latency, and scalability of the controllers on the physical server (bare-metal) with Hyper-Threading enabled. Second, we performed the same tests on ONOS and OpenDaylight on the physical server without Hyper-Threading. Finally, in the last phase, we constructed a virtual testbed based on OpenStack on top of our physical infrastructure, and examined ONOS and OpenDaylight performance. In total, we performed 36 tests (12 tests in each phase). In presenting the results, we combined the outcomes from the tests in each phase into one graph to facilitate comparison of results and avoid having repetitive figures.

The parameters for the throughput, latency, and scalability tests are summarized in Table 4.1, Table 4.2, and Table 4.3, respectively. Also, for the sake of brevity, we do not discuss the configuration of controllers for the performance tests in this chapter. However, Appendix A 
provides all the configuration settings.

Table 4.1: Parameters for throughput tests

\begin{tabular}{|c|c|c|}
\hline CPU Threads & Switches & Hosts \\
\hline 16 & $1-512$ & $100 \mathrm{~K}$ \\
\hline 16 & 16 & $1-1 \mathrm{M}$ \\
\hline
\end{tabular}

Table 4.2: Parameters for latency tests

\begin{tabular}{|c|c|c|}
\hline CPU Threads & Switches & Hosts \\
\hline 16 & $1-512$ & $100 \mathrm{~K}$ \\
\hline 16 & 16 & $1-1 \mathrm{M}$ \\
\hline
\end{tabular}

Table 4.3: Parameters for thread scalability tests

\begin{tabular}{|c|c|c|}
\hline CPU Threads & Switches & Hosts \\
\hline $1-16$ & 16 & $100 \mathrm{~K}$ \\
\hline $1-16$ & 16 & $100 \mathrm{~K}$ \\
\hline
\end{tabular}

It is worth noting that for all experiments (with 16 threads) we use the threads from both CPU sockets (first 12 threads from socket 1, and last 4 threads from socket 2). 


\subsection{Throughput Results}

In this section, we present the results of throughput tests on ONOS and OpenDaylight. We vary the number of connected switches as well as the number of hosts in the network. In the first test, to evaluate the impact of the number of connected switches on the throughput, all other parameters are fixed (e.g., duration of the test, the number of loops, the number of CPU threads, and the number of hosts). We conducted the first test with different number of switches and kept other parameters fixed. For the second throughput test, we varied the number of hosts, while keeping all other parameters fixed.

Figures 4.1 and 4.2 present the average throughput results for ONOS and OpenDaylight, respectively.

In Figure 4.1, throughput (in responses per second) is plotted versus the number of switches managed by the controller. The horizontal axis indicates the number of switches, which ranges from 1 to 512, on a logarithmic scale. The vertical axis of the graph indicates ONOS's average throughput, in responses per second. The vertical scale ranges from 0 to 1.8 million, on a linear scale. The error bars around each point denote one standard deviation above and below the mean. The mean and standard deviation are calculated based on five replications of the benchmark test.

Figure 4.1 shows that the throughput for ONOS scales very well. There is a steady increase in throughput for 1 to 16 switches, at which point it reaches 1.4 million responses/sec on the physical host with HT enabled. On the physical host with HT, ONOS's average throughput is around $200 \mathrm{~K}$ responses/sec with one switch. The standard deviation at this data point is $20 \mathrm{~K}$ responses/sec. ONOS's average throughput is about $255 \mathrm{~K}(\mathrm{resp} / \mathrm{s}), 440 \mathrm{~K}$ $(\mathrm{resp} / \mathrm{s}), 795 \mathrm{~K}(\mathrm{resp} / \mathrm{s})$, and 1.43M (resp/s) with 2, 4, 8, and 16 switches, respectively. The standard deviations for these data points are moderately larger and are about 30K (resp/s). Scaling up the number of switches beyond 16 does not significantly change the throughput for ONOS. 


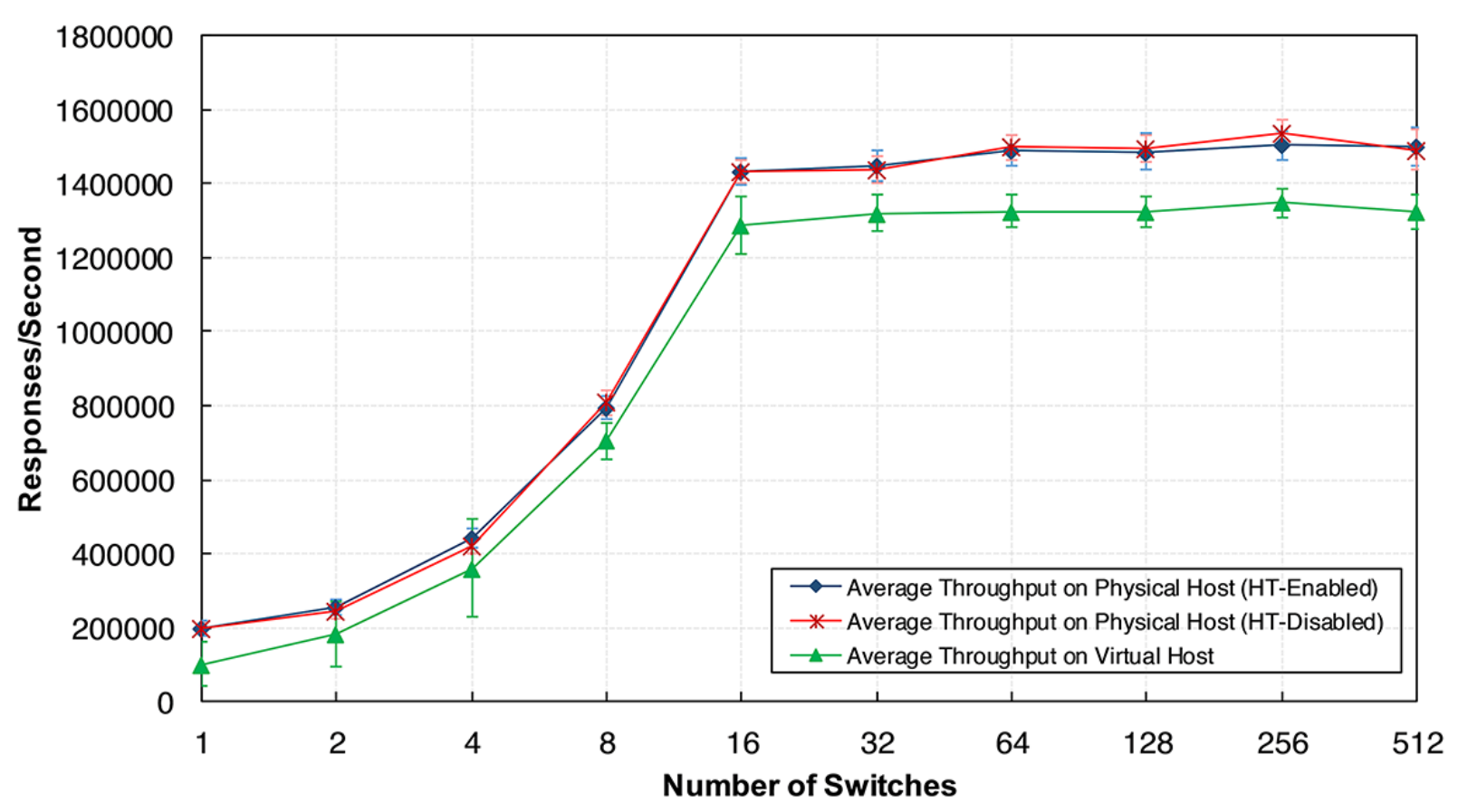

Figure 4.1: ONOS average throughput with different number of switches

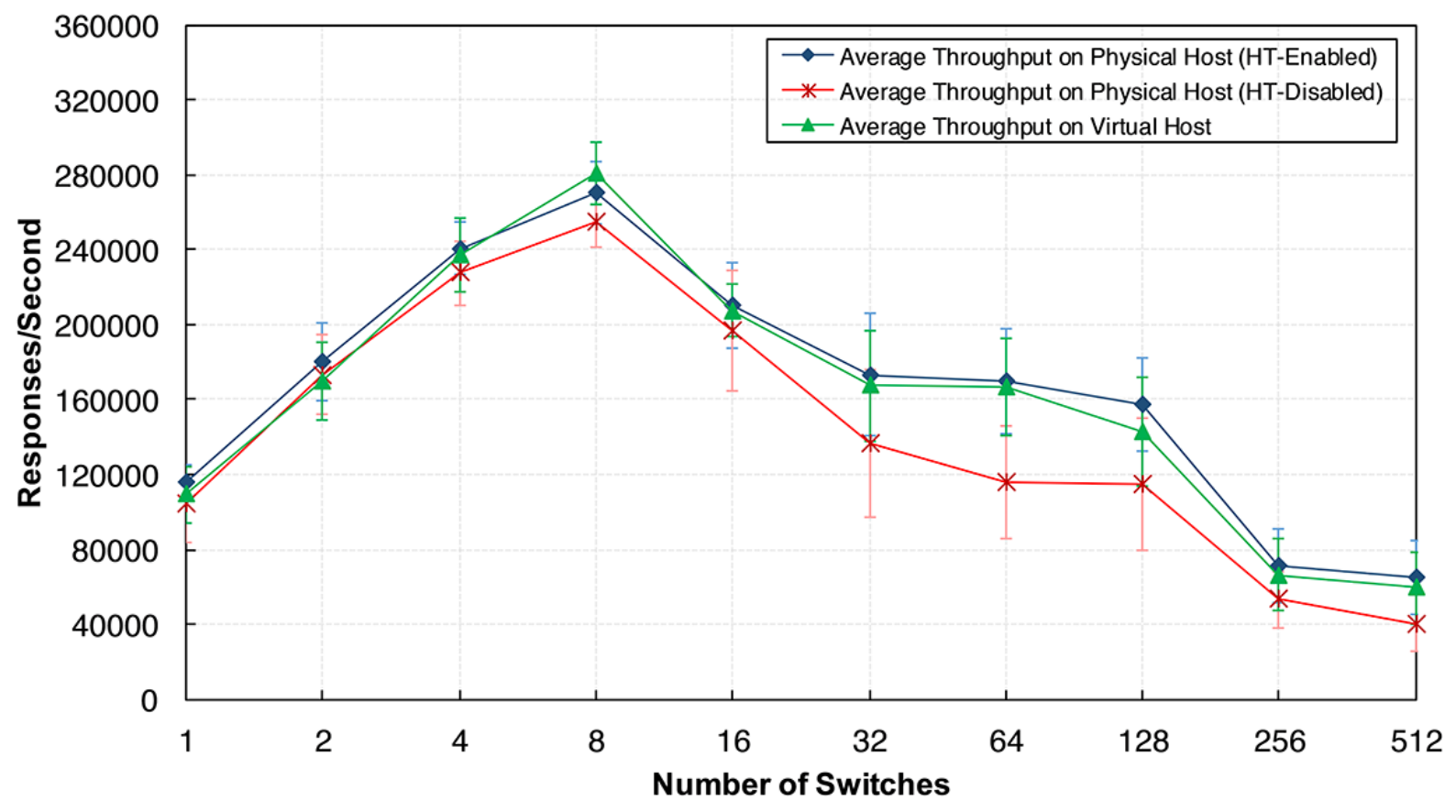

Figure 4.2: OpenDaylight average throughput with different number of switches 
As shown in Figure 4.1, the throughput enters a saturation plateau (1.5 million responses/sec) with $64,128,256$, and 512 switches. The error bars indicate the standard deviation for the mentioned data points is around 40K (resp/s). We observed a growth in the standard deviation as the number of switches increases.

For ONOS, turning Hyper-Threading off still provides the same average throughput as Hyper-Threading enabled. Also, the error bars are similar in this test with and without HT.

When using the virtual host, ONOS's throughput is roughly $13.5 \%$ lower than on the physical host. This performance gap indicates the impact of virtualization overhead on ONOS's throughput. With one switch, ONOS delivers $100 \mathrm{~K}$ responses/sec on the virtual host. The standard deviation at this data point is about 40K (resp/s). The average throughput results with 2, 4, 8 and 16 switches are $185 \mathrm{~K}(\mathrm{resp} / \mathrm{s}), 360 \mathrm{~K}(\mathrm{resp} / \mathrm{s}), 705 \mathrm{~K}$ (resp/s) and 1.3M (resp/s), respectively. Similar to throughput results on the physical host, ONOS's throughput on the virtual host doesn't change significantly with more than 16 switches.

As discussed above, the throughput trends for the physical and virtual hosts are similar, which indicates that ONOS behaves consistently regardless of the underlying infrastructure.

In our experiment, the throughput of ONOS increases until the number of switches reaches the number of threads used to run the controller (16 threads). The observed trend matches the behavior of other multi-threaded controllers that have been studied in the past. As mentioned in an earlier benchmarking study 83 for multi-threaded controllers, having more connected switches typically leads to better utilization of CPU threads until the number of switches exceeds the number of available threads.

Table 4.4 compares ONOS's average throughput in our study with prior work [80]. A comparison of these results shows that ONOS's performance has dramatically improved. In particular, the throughput doesn't degrade after reaching its peak performance. The authors of [80] conducted an experimental study on several SDN controllers including ONOS (Blackbird release). They examined ONOS's throughput with 8, 16, 64, and 100 switches. 
The highest throughput that they achieved is around $500 \mathrm{~K}$ responses/sec with 8 switches. Since they used $8 \mathrm{CPU}$ threads, increasing the number of switches beyond 8 did not lead to higher throughput. For ONOS's throughput, they achieved 200K (resp/s), 300K (resp/s) and $200 \mathrm{~K}$ (resp/s) with 16, 64, and 100 switches, respectively. In their experiments, ONOS's throughput declined after its peak performance with 8 switches. Their results indicate that ONOS had a performance bottleneck in the throughput test. However, our results don't show the throughput decline with increasing the number of switches. This indicates that the issue has been resolved in the Goldeneye release. It should be noted that the authors of 80 ] ran their experiments on a single server equipped with one Intel Core i7-3630QM CPU (8 cores @ 2.40GHZ) and 16 GB RAM.

Table 4.4: ONOS throughput comparison with prior work $\mid 80$

\begin{tabular}{|c|c|c|}
\hline \# Switches & Avg. TP with Blackbird $[80$ & Avg. TP with Goldeneye (Our Study) \\
\hline 8 & $400-500 \mathrm{~K}(\mathrm{resp} / \mathrm{s})$ & $800 \mathrm{~K}(\mathrm{resp} / \mathrm{s})$ \\
\hline 16 & $200 \mathrm{~K}(\mathrm{resp} / \mathrm{s})$ & $1.43 \mathrm{M}(\mathrm{resp} / \mathrm{s})$ \\
\hline 32 & $250 \mathrm{~K}(\mathrm{resp} / \mathrm{s})$ & $1.44 \mathrm{M}(\mathrm{resp} / \mathrm{s})$ \\
\hline 64 & $300 \mathrm{~K}(\mathrm{resp} / \mathrm{s})$ & $1.5 \mathrm{M}(\mathrm{resp} / \mathrm{s})$ \\
\hline
\end{tabular}

Figure 4.2 illustrates the average throughput of OpenDaylight based on number of switches. The graph is in the same format as Figure 4.1, except that the vertical axis scale is from 0 to $360 \mathrm{~K}$, rather than to $1.8 \mathrm{M}$.

OpenDaylight's highest throughput is achieved with 8 connected switches. At this point, the throughput averages $270 \mathrm{~K}$ responses/sec on the physical host with HT, and $255 \mathrm{~K}$ responses/sec on the physical host without HT. On the virtual host, the average is $280 \mathrm{~K}$ responses/sec.

For a single switch, OpenDaylight's average throughput on the physical host with HT is $120 \mathrm{~K}(\mathrm{resp} / \mathrm{s})$. Throughput grows steadily at first, reaching $180 \mathrm{~K}$ (resp/s), 240K (resp/s), 
and $270 \mathrm{~K}(\mathrm{resp} / \mathrm{s})$ with 2,4 , and 8 switches, respectively. However, there is a drastic throughput reduction as the number of switches is increased beyond 8. As shown in Figure 4.2. OpenDaylight's average throughput with 16 switches is $210 \mathrm{~K}$ (resp/s), and 170(resp/s) for 32 switches. The throughput declines slowly beyond 32 switches, reaching $157 \mathrm{~K}$ (resp/s) with 128 switches. OpenDaylight's throughput drops sharply after this point. With 256 and 512 switches, the results are about $70 \mathrm{~K}$ responses/sec.

For OpenDaylight, the throughput with and without HT are not the same. OpenDaylight has its highest throughput on the physical host when Hyper-Threading is enabled. For instance, the throughput on the physical host with HT is $157 \mathrm{~K}$ (resp/s) for 128 switches, while it is $114 \mathrm{~K}(\mathrm{resp} / \mathrm{s})$ without HT. OpenDaylight's standard deviations in the throughput test are comparatively larger when HT is disabled. For instance, with 32 switches, the standard deviation is about $23 \mathrm{~K}(\mathrm{resp} / \mathrm{s})$ when HT is enabled. Without HT, the standard deviation is about $32 \mathrm{~K}(\mathrm{resp} / \mathrm{s})$ for the same number of switches.

OpenDaylight's throughput on the virtual host is only slightly lower than its throughput on the physical host with HT. OpenDaylight's average throughput on the virtual host for a single switch is $110 \mathrm{~K}(\mathrm{resp} / \mathrm{s})$, and rises steadily to $280 \mathrm{~K}(\mathrm{resp} / \mathrm{s})$ for 8 switches. The decline in throughput beyond 8 switches is similar to that on the physical host. OpenDaylight's average throughput on the virtual host is typically higher than the throughput on the physical host without HT.

Comparing OpenDaylight's average throughput on the physical host (with HT) with that for the virtual host, OpenDaylight's performance change is smaller than ONOS's performance change. As an example, ONOS's average throughput, with 16 switches, is $1.43 \mathrm{M}$ (resp/s) on the physical host with HT, and $1.28 \mathrm{M}(\mathrm{resp} / \mathrm{s})$ on the virtual host. On the other hand, OpenDaylight's throughput results for 16 switches are $210 \mathrm{~K}(\mathrm{resp} / \mathrm{s})$ and $207 \mathrm{~K}$ (resp/s), on the physical host with HT, and on the virtual host, respectively.

The results clearly indicate that ONOS outperforms OpenDaylight in the throughput 
test. ONOS shows very stable performance, and about 10 times better overall throughput (for 32 switches) than OpenDaylight.

As shown in Figure 4.2, OpenDaylight's throughput declines when the number of switches exceeds 8. This behavior of OpenDaylight has been mentioned in the literature [60]. We observed some throughput test failures with 256 and 512 switches. Even though we managed to have some successful tests with 512 switches, OpenDaylight responded with zero in many of our tests (Figure 4.3b). We discarded the failed tests and calculated the average throughput and overall standard deviation based on the successful tests.

In [60], the authors discovered that OpenDaylight is unable to respond to many of the latency tests, and the failure ratio increases with more switches. We discuss this in further detail in the latency section.

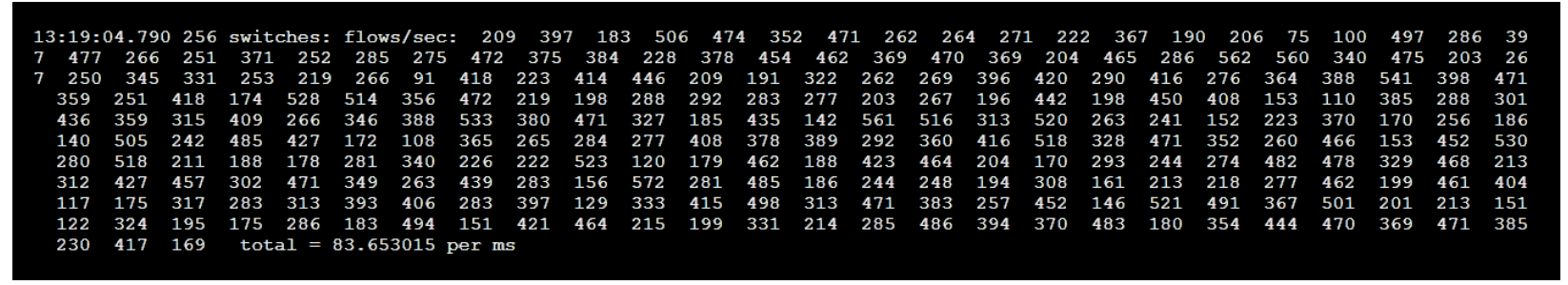

(a) OpenDaylight successful test with 256 switches

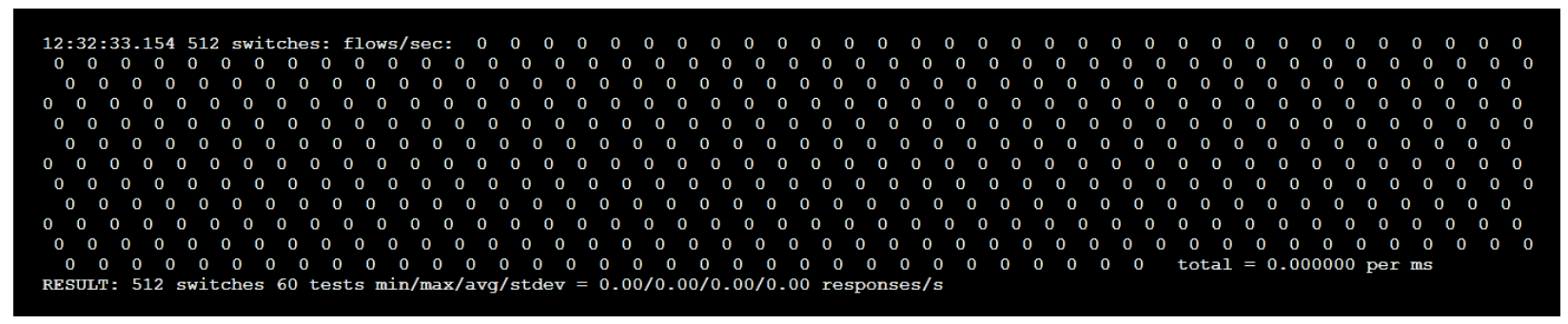

(b) OpenDaylight failed test with 512 switches

Figure 4.3: Example of OpenDaylight throughput tests with 256 and 512 switches 
Table 4.5: OpenDaylight throughput failed tests

\begin{tabular}{|c|c|c|}
\hline \# Switches & Total Tests & Failed Test \\
\hline 256 & 300 & $20 \%$ \\
\hline 512 & 300 & $40 \%$ \\
\hline
\end{tabular}

Table 4.5 summarizes OpenDaylight's throughput test failure ratio in our study. We did not observe any throughput test failures with 128 switches or fewer.

We summarize OpenDaylight's average throughput in our study in Table 4.6, and compare it with average and maximum throughput from [60]. The authors conducted a preliminary experimental evaluation on throughput and latency of OpenDaylight and Floodlight controllers. They used one HP Compaq Pro 6300s PC to run the controller (equipped with one Intel Core i5 CPU and 8 GB RAM), and four servers with a similar specification to run multiple instances of Cbench. The average throughput achieved in their study is extremely low compared to our result. Comparing the results shows that OpenDaylight's performance has significantly improved in Beryllium-SR2 (our study) compared to Hydrogen release used in their study.

Table 4.6: OpenDaylight throughput comparison with prior work 60

\begin{tabular}{|c|c|c|c|}
\hline \# Switches & Avg. TP in $\mid 60$ & Max. TP in $60 \mid$ & Avg. TP in Our Study \\
\hline 8 & $270(\mathrm{resp} / \mathrm{s})$ & $4.8 \mathrm{~K}(\mathrm{resp} / \mathrm{s})$ & $270 \mathrm{~K}(\mathrm{resp} / \mathrm{s})$ \\
\hline 16 & $33(\mathrm{resp} / \mathrm{s})$ & $350(\mathrm{resp} / \mathrm{s})$ & $210 \mathrm{~K}(\mathrm{resp} / \mathrm{s})$ \\
\hline 32 & $34(\mathrm{resp} / \mathrm{s})$ & $900(\mathrm{resp} / \mathrm{s})$ & $173 \mathrm{~K}(\mathrm{resp} / \mathrm{s})$ \\
\hline
\end{tabular}

It is worth noting that each SDN controller might have a particular configuration for the throughput test. Through discussions with OpenDaylight developers, we learned that running the learning switch application is not recommended for the throughput test with Cbench. Having this feature enabled can slow down the test. Instead, it is recommended 
to install a feature (odl-openflowplugin-drop-test) that is designed for the throughput test. The learning switch application has frequently been used for throughput tests in prior work [48] [81] [90], since it is a lightweight application with reasonable memory usage. More importantly, it allows testing the I/O throughput of the controller. In essence, the learning switch responds to the packet_in messages that Cbench sends toward the controller. However, OpenDaylight still allows the test to run without the learning switch installed.

As discussed in Chapter 3, the throughput test evaluates the controller's capacity to handle packet_in messages being sent by switches toward the controller. In other words, the throughput test measures the number of packet_in messages that can be processed per unit time. In single thread controllers, such as Ryu and POX, the correlation between throughput and latency is quite straightforward. Specifically, throughput is equal to 1/latency. However, in multi-threaded controllers, such as ONOS and OpenDaylight, throughput is greater than 1 /latency since packets can be processed in parallel. So the throughput test gives an indication of multi-threaded controller efficiency, as well as I/O capacity.

For I/O modules, ONOS (Goldeneye) and OpenDaylight (Beryllium-SR2) both use the Netty [23] framework. Netty is a high-performance Non-blocking I/O (NIO) networking library for development of Java-based network applications. In essence, Netty was designed to streamline network programming (e.g., UDP and TCP socket servers). Netty allows high throughput I/O communications and has low latency [23]. It has been used in other controllers as well, such as Floodlight [44] 82]. Interestingly, the Netty version that OpenDaylight uses (4.0.33.Final) is newer than the version that ONOS employs (3.10). Floodlight controller (version 1.1 or above), which also uses Netty, showed an average throughput of 1 - 1.5 million responses/sec in various studies [44 [60 [76 83 [87 85. The results are very close to ONOS average throughput numbers, and provides an indication of the maximum throughput deliverable by Netty.

As a next step, we conducted a follow-up experiment on the OpenDaylight throughput 
and assigned more CPU threads (24) to the controller, compared to the initial test with 16 threads.

Figure 4.4 shows slightly better throughput compared to the initial result with 16 threads. However, the trend is still the same, and throughput declines when increasing the number of switches to more than 8. We discuss OpenDaylight's throughput issue in further detail in the next chapter.

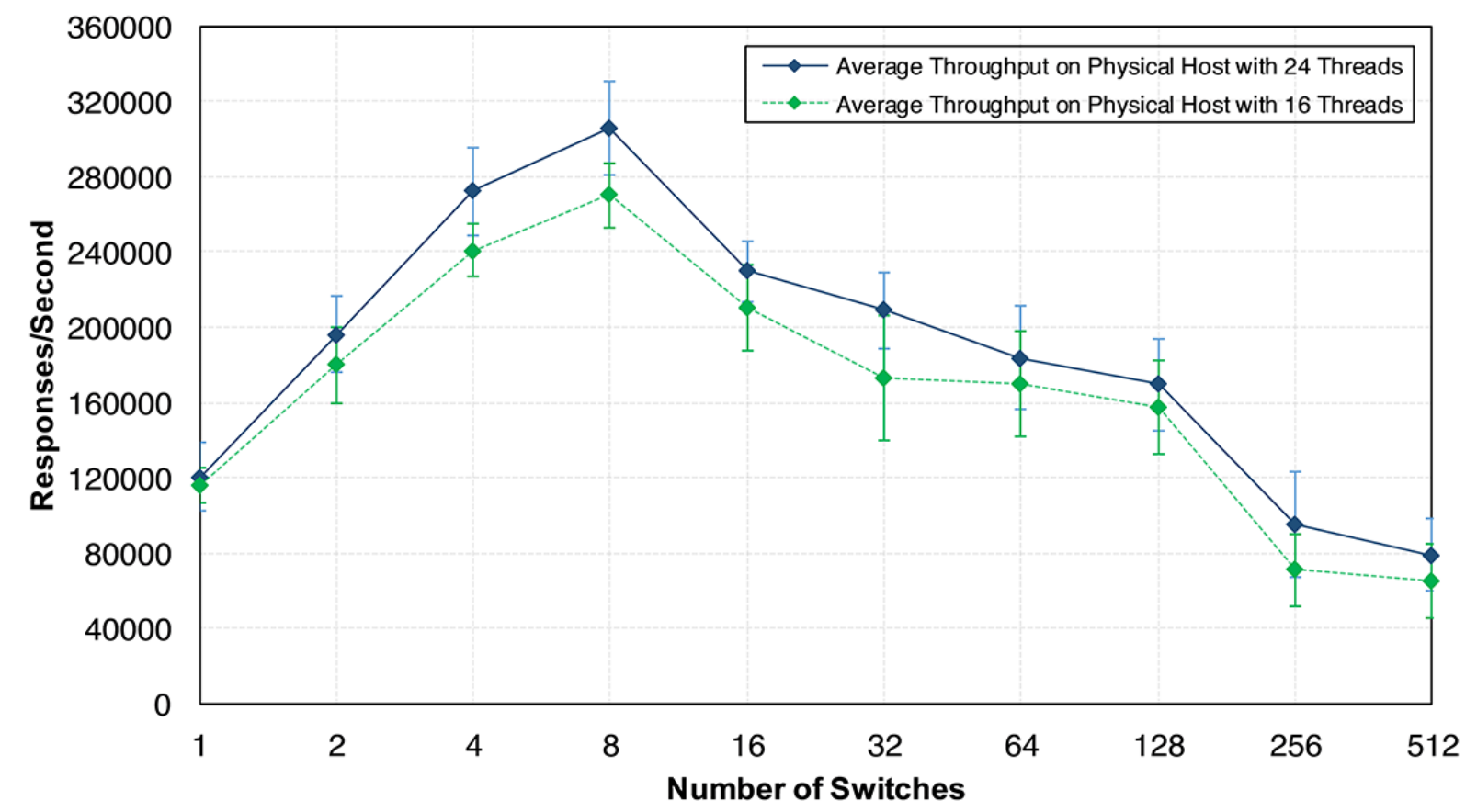

Figure 4.4: OpenDaylight average throughput with different number of switches using 16 and 24 threads

We compared the throughput results of ONOS and OpenDaylight from our study with other controllers in previous work. The authors of [83] conducted an experimental evaluation of throughput and latency for some open-source controllers, namely Beacon, NOX, POX, MUL, Floodlight, Ryu, and Maestro. They used two servers to conduct their experiments. The server used to run the controllers had two Intel Xeon E5645 CPUs (6 cores @ 2.40GHz) and 48 GB RAM. 
Table 4.7 summarizes the results, and compares the throughput of ONOS and OpenDaylight with other open-source multi-threaded controllers (presented in alphabetical order).

Table 4.7: ONOS and OpenDaylight throughput comparison with [83]

\begin{tabular}{|c|c|c|c|c|c|c|}
\cline { 2 - 7 } \multicolumn{1}{c|}{} & \multicolumn{6}{c|}{ Throughput (resp/s) } \\
\hline \# Switch & Beacon & Floodlight & Maestro & MUL & ONOS & OpenDaylight \\
\hline 1 & $1.2 \mathrm{M}$ & $300 \mathrm{~K}$ & $2 \mathrm{M}$ & $600 \mathrm{~K}$ & $200 \mathrm{~K}$ & $120 \mathrm{~K}$ \\
\hline 8 & $5.3 \mathrm{M}$ & $1.2 \mathrm{M}$ & $3.7 \mathrm{M}$ & $2.5 \mathrm{M}$ & $800 \mathrm{~K}$ & $270 \mathrm{~K}$ \\
\hline 16 & $6.2 \mathrm{M}$ & $1.5 \mathrm{M}$ & $3.9 \mathrm{M}$ & $2.8 \mathrm{M}$ & $1.43 \mathrm{M}$ & $207 \mathrm{~K}$ \\
\hline 32 & $6.4 \mathrm{M}$ & $1.5 \mathrm{M}$ & $4 \mathrm{M}$ & $2.7 \mathrm{M}$ & $1.43 \mathrm{M}$ & $167 \mathrm{~K}$ \\
\hline 64 & $6.1 \mathrm{M}$ & $1.6 \mathrm{M}$ & $3.6 \mathrm{M}$ & $2.6 \mathrm{M}$ & $1.49 \mathrm{M}$ & $166 \mathrm{~K}$ \\
\hline 128 & $6 \mathrm{M}$ & $1.5 \mathrm{M}$ & $2 \mathrm{M}$ & $2.5 \mathrm{M}$ & $1.49 \mathrm{M}$ & $157 \mathrm{~K}$ \\
\hline 256 & $6 \mathrm{M}$ & $1.5 \mathrm{M}$ & $100 \mathrm{~K}$ & $2.5 \mathrm{M}$ & $1.53 \mathrm{M}$ & $70 \mathrm{~K}$ \\
\hline
\end{tabular}

As shown in Table 4.7, Beacon has the highest throughput among multi-threaded controllers. Following Beacon, Maestro and MUL have higher throughput compared to the rest of the controllers. Maestro showed drastic throughput degradation in their study. The authors attributed this to Maestro's packet distribution algorithm, which is round-robin. Floodlight's throughput results are similar to ONOS, since they both use identical network I/O libraries, and are Java-based controllers. OpenDaylight's throughput performance is lower than all other controllers.

Comparing the throughput results of our study with the throughput of other multithreaded controllers reveals that ONOS is the only controller that does not show throughput degradation when increasing the number of switches. However, ONOS still has room for improvement, since its throughput is about average compared to other open-source controllers.

Figures 4.5 and 4.6 demonstrate the average throughput of ONOS and OpenDaylight, respectively, based on the number of hosts (different MAC address) in the network. 
In Figure 4.5, the horizontal axis shows the number of hosts in the network. The logarithmic scale of this axis goes from 1 to 1 million. The vertical axis of the graph indicates ONOS's average throughput, on a linear scale. The error bars correspond to one standard deviation above and below the mean value for each point.

Figure 4.5 shows that the throughput of ONOS is always between 1.4 million (resp/s) and 1.5 million (resp/s) on the physical host. ONOS showed similar performance with and without HT. For instance, the average throughput with 1 host is 1.44 million (resp/s) on the physical host with and without HT.

For ONOS, the average throughput on the virtual host is always between 1.3 million (resp/s) and 1.35 million (resp/s). The gap between throughput on the physical host and virtual host in Figure 4.5 is quantitatively similar to that in Figure 4.1 (about 13\%). Overall, ONOS's throughput is about $150 \mathrm{~K}(\mathrm{resp} / \mathrm{s})$ lower on the virtual host. The standard deviations for most of the data points are around $50 \mathrm{~K}(\mathrm{resp} / \mathrm{s})$ on the physical host (with HT and without HT) and virtual host.

Figure 4.6 shows the average throughput of OpenDaylight based on the number of hosts in the network. The format of the graph is the same as Figure 4.5, except that the vertical axis scale ranges from 0 to $320 \mathrm{~K}$.

As shown in Figure 4.6, OpenDaylight's throughput in this test is not the same as ONOS. The average throughput of OpenDaylight is around 200K (resp/s) on the physical host with HT. Without HT, the throughput is lower for some data points. For example, with $10 \mathrm{~K}$ hosts, the throughput is $195 \mathrm{~K}(\mathrm{resp} / \mathrm{s})$. The standard deviations for throughput tests on the physical host without HT are comparatively larger than in the test on the physical host with HT. For most of the data points, the standard deviations are $35 \mathrm{~K}(\mathrm{resp} / \mathrm{s})$.

On the other hand, the throughput of OpenDaylight on the virtual host is similar to the results on the physical host with HT. The standard deviation for the throughput test on the virtual host is around $20 \mathrm{~K}(\mathrm{resp} / \mathrm{s})$. 


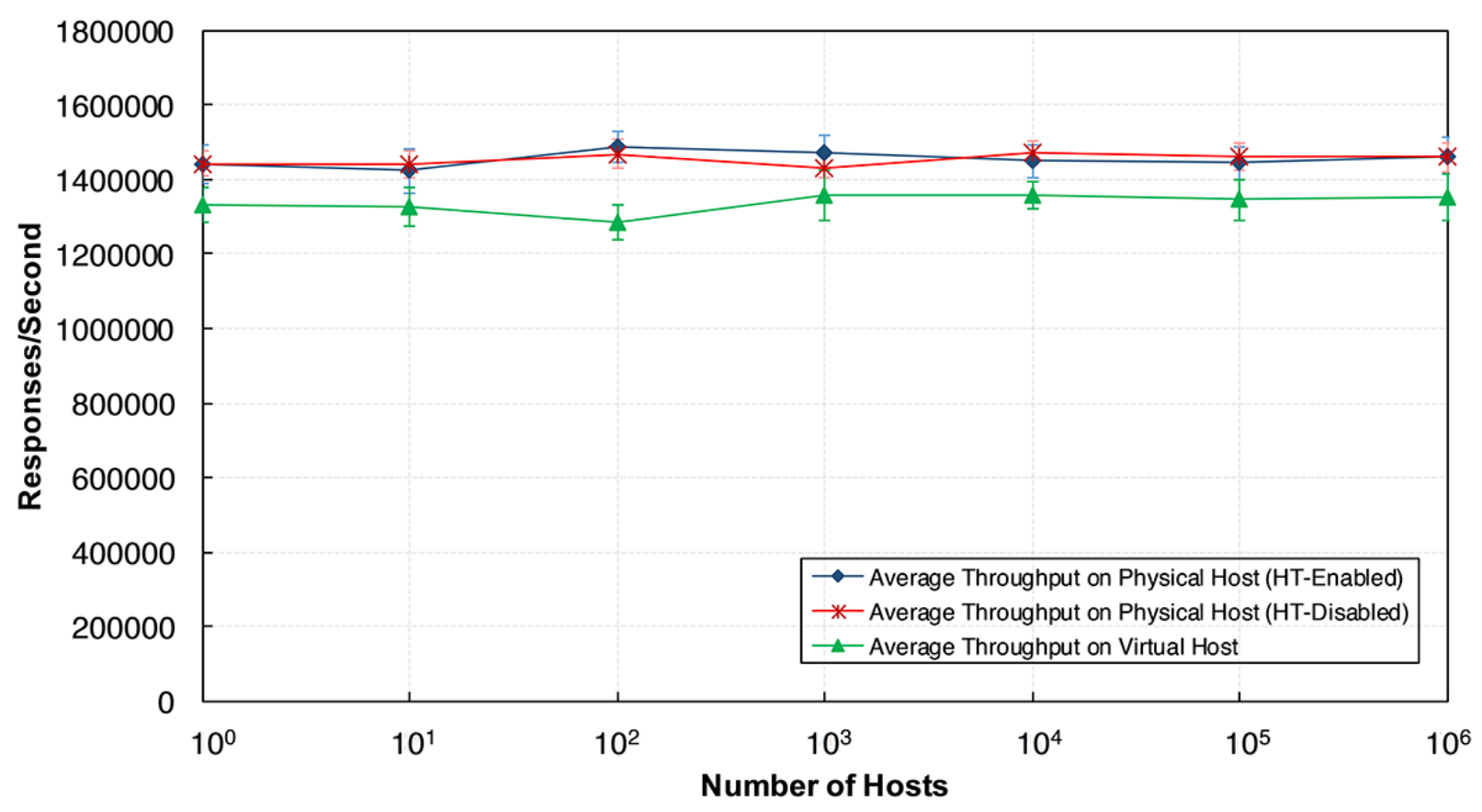

Figure 4.5: ONOS average throughput with different number of hosts

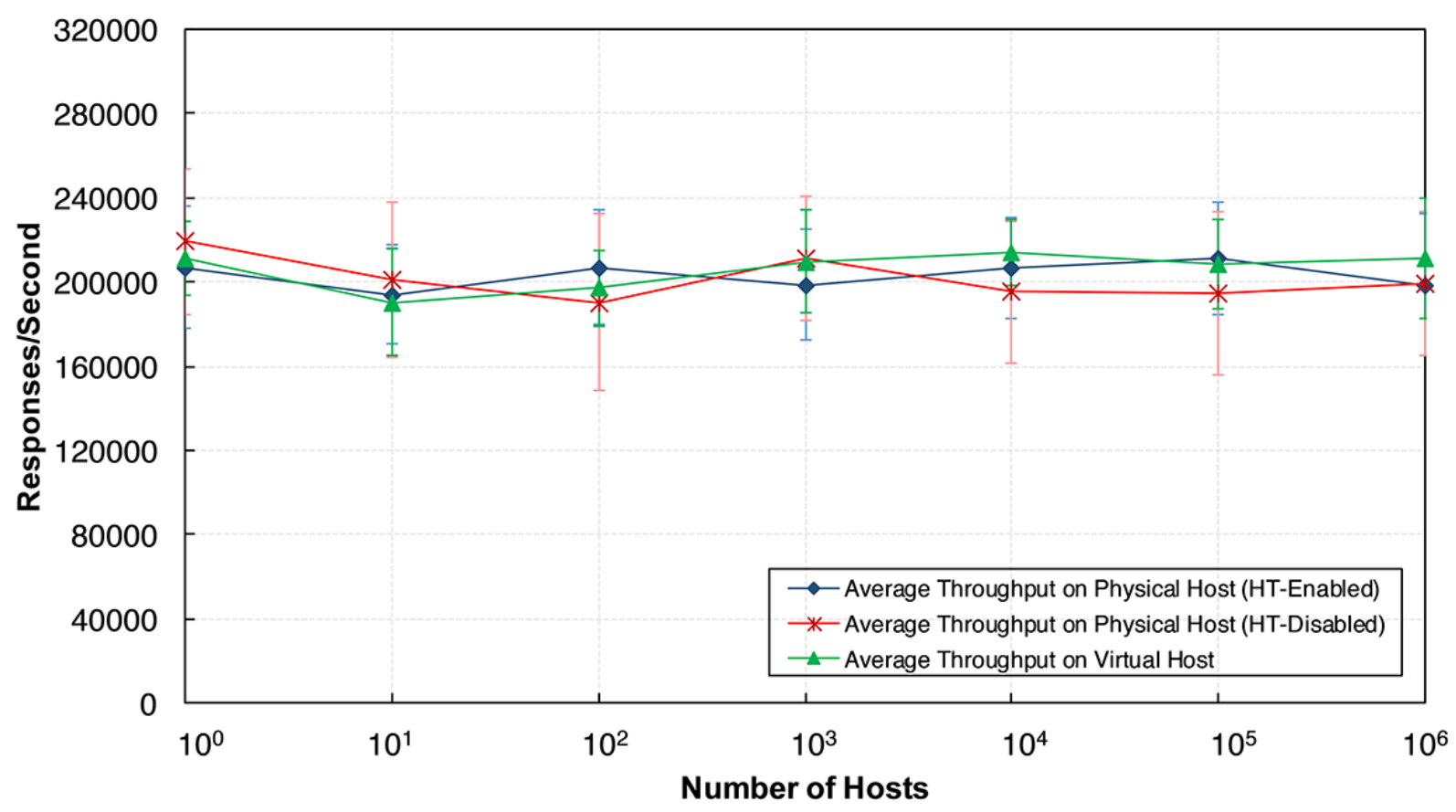

Figure 4.6: OpenDaylight average throughput with different number of hosts 
The results of this test demonstrate that the number of hosts in the network has negligible impact on the performance of ONOS and OpenDaylight. Comparing our result in this test with an earlier study on OpenFlow controllers [83], the behavior of ONOS and OpenDaylight in this test is consistent with the behavior of Floodlight, MUL, NOX, POX, and Ryu in their work, for which the number of hosts in the network has negligible influence on the throughput. In [83, Beacon and Maestro showed throughput degradation when increasing the number of hosts. In particular, Beacon's throughput declined when the number of hosts exceeded 1 million. For Maestro, the throughput degradation occurred when the number of hosts increased beyond $100 \mathrm{~K}$. The authors determined that implementation of the lookup table in the Learning Switch application caused this throughput decline in these controllers [83].

\subsection{Latency Results}

In this section, we show the results of latency tests on ONOS and OpenDaylight. We vary the number of connected switches, as well as the number of hosts in the network. Similar to the throughput tests, we kept all parameters fixed except the number of switches in the first test, and the number of hosts in the second test.

Figure 4.7 and Figure 4.8 depict the average flow setup latency of ONOS and OpenDaylight, respectively, versus the number of connected switches.

In Figure 4.7, the horizontal axis of the graph shows the number of switches, using a logarithmic scale. The vertical axis of the graph shows ONOS's average flow setup latency, in microseconds. The error bars represent one standard deviation above and below data points, with the mean and standard deviations calculated using 5 runs. The error bars in this graph are barely visible, since they are very small.

Both controllers demonstrate structurally similar trends in this test. As the number of connected switches increases, the flow setup latency decreases. 


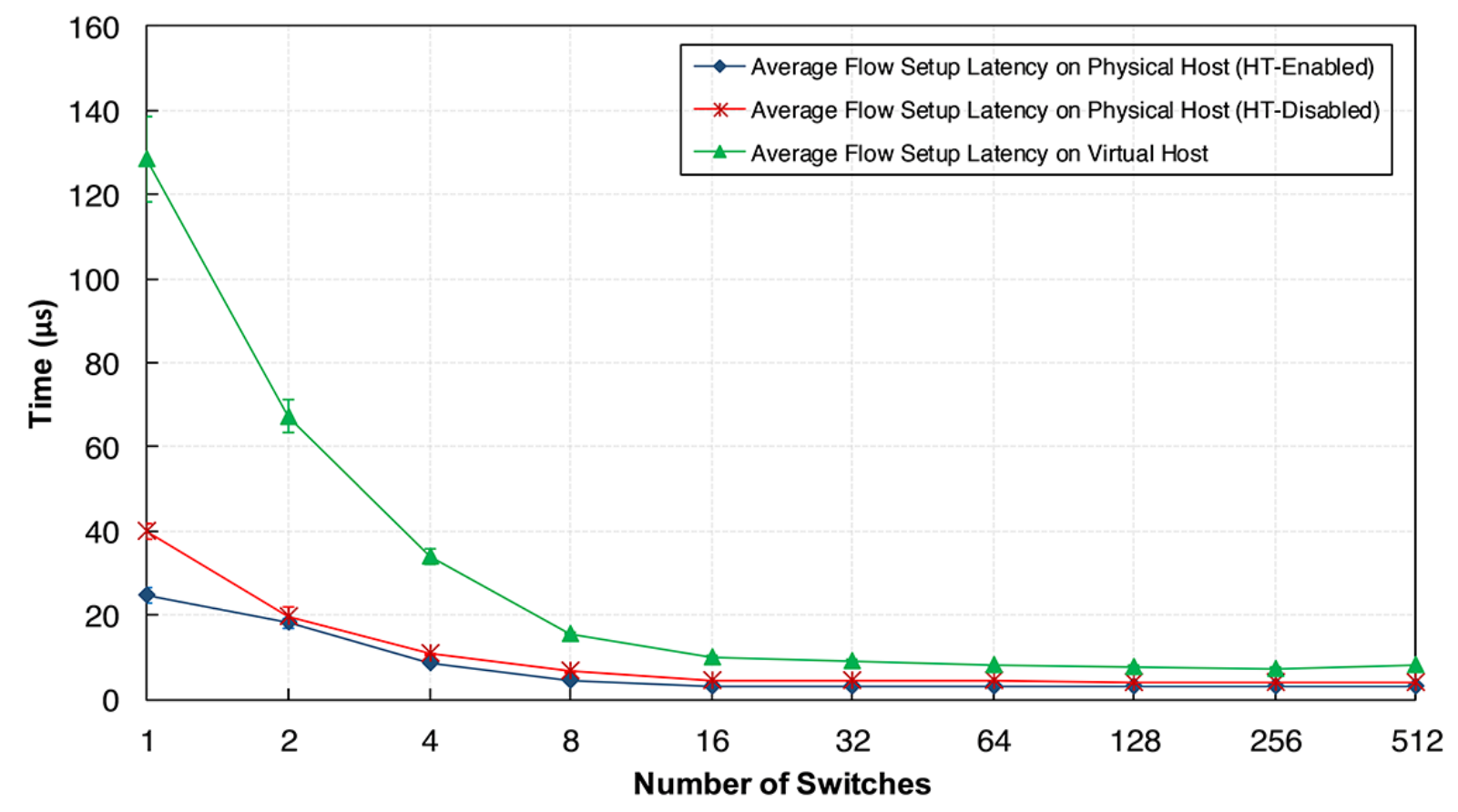

Figure 4.7: ONOS average flow setup latency with different number of switches

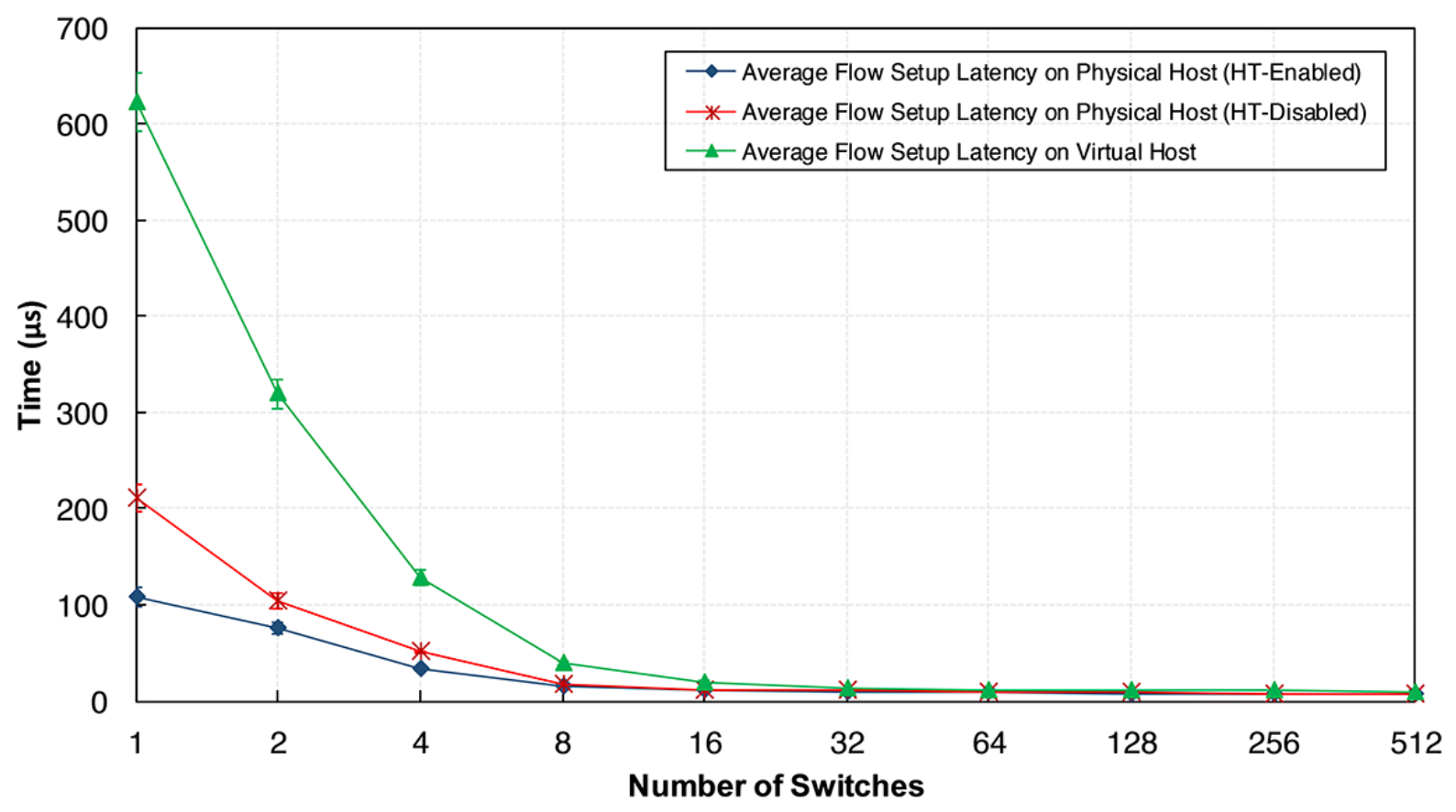

Figure 4.8: OpenDaylight average flow setup latency with different number of switches 
As discussed in Chapter 3 , in the latency test, each emulated switch in Cbench sends a packet_in message to the controller, and waits to receive the packet_out message (response from controller). Cbench measures the delay between the packet_in message and the packet_out message to calculate flow setup latency of the controller. By increasing the number of switches, the rate of packet_in messages in Cbench increases. As a result, the controller receives more packet_in messages, which are then processed in parallel. This results in receiving more packet_out messages from the controller in the given time, and subsequently decreases flow setup latency. This reflects the Task Batching model implemented in each controller.

Task Batching is a method used in multi-threaded controllers to allocate already received packet_in messages to the worker threads for processing. Task Batching is one of the key features in multi-threaded controllers that impacts the latency [81]. That being said, flow setup latency in the multi-threaded controllers does not increase when scaling up the number of switches.

It is worth noting that Cbench only establishes one TCP connection to the controller for all emulated switches. Thus, it gathers aggregated statistics for all switches, but not for each switch individually [58]. As a result, it is not possible to have a granular analysis (e.g., determine per-switch latency).

The observed trend in the latency test, for both ONOS and OpenDaylight, matches the behavior of other multi-threaded controllers that have been studied before [60] [80] [87.

Figure 4.7 shows that ONOS's average flow setup latency for one switch is $24 \mu$ s on the physical host with HT. For 2 switches, latency is about $20 \mu \mathrm{s}$. The average flow setup latency with 32 switches (and more) is 3 to $4 \mu$ s on the physical host.

On the other hand, without HT, the average flow setup latency with 1 switch is about $40 \mu$ s. However, with 2 switches (and more) the results are similar to the results with HT. Our latency tests on the virtual host indicate ONOS's average flow setup latency with 
one switch is $128 \mu \mathrm{s}$, which is high compared to the flow setup latency with one switch on the physical host. However, increasing the number of switches yielded lower latency. For example, with $2,4,8$, and 16 switches the flow setup latencies are $67 \mu \mathrm{s}, 33 \mu \mathrm{s}, 15 \mu \mathrm{s}$, and $10 \mu \mathrm{s}$, respectively. The latency for 32 switches (and more) is 7 to $9 \mu \mathrm{s}$.

Figure 4.8 presents the average flow setup latency of OpenDaylight. In Figure 4.8, the vertical axis scale is from 0 to 700 microseconds. Similar to Figure 4.7, the error bars in this graph are barely visible.

For OpenDaylight, when HT is enabled, the average flow setup latency with 1 switch is $108 \mu \mathrm{s}$. Adding more switches results in lower latency. For instance, with 2, 4, and 8 switches, the OpenDaylight flow setup latencies are $76 \mu \mathrm{s}, 33 \mu \mathrm{s}$, and $10 \mu \mathrm{s}$, respectively. The average flow setup latency with 32 switches (and more) is about $7 \mu$ s on the physical host.

Similar to ONOS, we observed slightly better performance when HT is enabled. Without HT, the average flow setup latency with 1 switch is $200 \mu \mathrm{s}$. Adding the second switch decreases flow setup latency to $100 \mu \mathrm{s}$. With 4 connected switches, we observed the flow setup latency is about $50 \mu \mathrm{s}$. The average flow setup latency with 32 switches (and more) is about $8 \mu \mathrm{s}$.

OpenDaylight's flow setup latency on the virtual host, with one switch, is about 600 $\mu$ s. Similar to ONOS, OpenDaylight's flow setup latency on the virtual host with 1 switch is about 6 times higher than latency on the physical host with HT. The latency values decrease with additional switches, up to 16 switches. With 2, 4, 8, and 16 switches, flow setup latencies are $318 \mu \mathrm{s}, 120 \mu \mathrm{s}, 35 \mu \mathrm{s}$, and $20 \mu \mathrm{s}$. The average flow setup latency with 32 switches (and more) is about $10 \mu$ s.

Overall, the average flow setup latency of ONOS is about half of OpenDaylight's latency. Moreover, we compared our result with a recent study, summarized in Table 4.8. OpenDaylight latency is about the same, but ONOS latency has improved by $50 \%$. 
Table 4.8: ONOS and OpenDaylight latency comparison with prior work 80|

\begin{tabular}{|c|c|c|c|c|}
\hline \# Switches & ONOS LT 80 & ONOS LT Our Work & ODL LT 80$]$ & ODL LT Our Work \\
\hline 8 & $8 \mu \mathrm{s}$ & $4.8 \mu \mathrm{s}$ & $6 \mu \mathrm{s}$ & $10 \mu \mathrm{s}$ \\
\hline 16 & $7 \mu \mathrm{s}$ & $4 \mu \mathrm{s}$ & $5 \mu \mathrm{s}$ & $8 \mu \mathrm{s}$ \\
\hline 32 & $7 \mu \mathrm{s}$ & $3.8 \mu \mathrm{s}$ & $7 \mu \mathrm{s}$ & $7 \mu \mathrm{s}$ \\
\hline 64 & $7 \mu \mathrm{s}$ & $3.7 \mu \mathrm{s}$ & $7 \mu \mathrm{s}$ & $7 \mu \mathrm{s}$ \\
\hline
\end{tabular}

For 8 and 16 connected switches, the latency values shown in the prior work are slightly better compared to ours. However, since the latency values for 32 and 64 switches are the same, the observed gap for 8 and 16 switches could be the result of the difference in loops per iteration, or rounds of experiment in the two studies. Also, it is possible that the prior work presents minimum obtained latency, instead of average.

Table 4.9: Comparing OpenDaylight latency failed tests ratio with prior work 60|

\begin{tabular}{|c|c|c|c|c|}
\hline \# Switches & Total Tests $\mid 60$ & Failed Test 60 & Our Total Tests & Our Failed Tests \\
\hline 8 & 80 & $20 \%$ & 300 & $0 \%$ \\
\hline 16 & 160 & $52 \%$ & 300 & $0 \%$ \\
\hline 32 & 320 & $58 \%$ & 300 & $0 \%$ \\
\hline 64 & N/A & N/A & 300 & $0 \%$ \\
\hline 128 & N/A & N/A & 300 & $0 \%$ \\
\hline 256 & N/A & N/A & 300 & $0 \%$ \\
\hline 512 & N/A & N/A & 300 & $0 \%$ \\
\hline
\end{tabular}

Table 4.9 summarizes OpenDaylight's latency test failure ratio in our study, and compares it with prior work [60]. The authors benchmarked throughput and latency of OpenDaylight and Floodlight controllers with 8, 16, and 32 switches. In their study, they used Hydrogen, 
the very first release of the OpenDaylight controller. As shown in Table 4.10, OpenDaylight provided unexpectedly high latency values in their work.

Table 4.10: OpenDaylight latency comparison with prior work $\mid 60\rfloor$

\begin{tabular}{|c|c|c|}
\hline \# Switch & Latency with Hydrogen $|60|$ & Latency with Beryllium-SR2 (Our Study) \\
\hline 8 & $18.18 \mathrm{~ms}$ & $10 \mu \mathrm{s}$ \\
\hline 16 & $37 \mathrm{~ms}$ & $8 \mu \mathrm{s}$ \\
\hline 32 & $66 \mathrm{~ms}$ & $7 \mu \mathrm{s}$ \\
\hline
\end{tabular}

We also compared the flow setup latency of ONOS and OpenDaylight in our study with the flow setup latency of some other controllers studied in previous work [99]. Each controller used only one thread, and the number of connected switches is one. Table 4.11 summarizes the flow setup latency of controllers in both studies.

The authors of [99] focused on performance evaluation of several well-known open-source controllers, namely Beacon, Floodlight, NOX, POX, and Ryu. Their study was designed to determine the performance of controllers with only one thread.

Table 4.11: ONOS and OpenDaylight flow setup latency comparison with 99

\begin{tabular}{|c|c|c|c|}
\hline \# Switch & Controller & Version & Latency $(\mu \mathrm{s})$ \\
\hline 1 & Beacon & 1.04 & 16.4 \\
\hline 1 & Floodlight & 0.90 & 22.2 \\
\hline 1 & NOX & 0.9 .2 & 17.9 \\
\hline 1 & POX & 0.2 .0 & 41.6 \\
\hline 1 & Ryu & 3.19 & 37 \\
\hline 1 & ONOS & Goldeneye & 24 \\
\hline 1 & OpenDaylight & Beryllium-SR2 & 108 \\
\hline
\end{tabular}


The authors ran their experiments on a single server equipped with one Intel Xeon E51620 (with 4 cores @ 3.6GHz). As shown in Table 4.11. Beacon has the lowest latency among all controllers. Its flow setup latency is $16.4 \mu$ s for one switch. Following Beacon, NOX, and Floodlight, ONOS has lower flow setup latency compared to the other controllers. ONOS's flow setup latency is $24 \mu$ s for one switch. On the other hand, OpenDaylight's flow setup latency with one switch is $108 \mu \mathrm{s}$, which is highest among all controllers listed above.

We also compared the latency results of ONOS and OpenDaylight in our study with previous work. The authors of [87] conducted a preliminary evaluation of some open-source controllers, namely Beacon, Floodlight, Maestro, NOX, OpenIRIS [16], and OpenMUL [17]. Table 4.12 summarizes the latency results of their work (presented in alphabetical order), and compares it with the latency of ONOS and OpenDaylight in our study. The author ran the controllers on a server equipped with one Intel Core i7-3537U CPU (2 cores @ $3.10 \mathrm{GHz}$ ) and 8 GB RAM.

Table 4.12: ONOS and OpenDaylight flow setup latency comparison with 87

\begin{tabular}{|c|c|c|c|c|c|c|c|c|}
\cline { 2 - 10 } \multicolumn{1}{c|}{} & \multicolumn{10}{c|}{ Latency $(\mu \mathrm{s})$} \\
\hline \# Switch & Beacon & Floodlight & Maestro & NOX & OpenIRIS & OpenMUL & ONOS & ODL \\
\hline 1 & 15 & 21 & 61 & 23 & 13 & 12 & 24 & 108 \\
\hline 8 & 7 & 9 & 15 & 9 & 5 & 10 & 4 & 10 \\
\hline 16 & 9 & 9 & 12 & 9 & 5 & 10 & 3 & 8 \\
\hline 32 & 7 & 9 & 11 & 9 & 5 & 10 & 3 & 7 \\
\hline 64 & 8 & 9 & 11 & 9 & 5 & 10 & 3 & 7 \\
\hline 128 & 8 & 14 & 11 & 9 & 5 & 10 & 3 & 7 \\
\hline
\end{tabular}

As shown in Table 4.12, for one switch, OpenMUL and OpenIRIS have the lowest flow setup latency among all controllers. ONOS latency with one switch is $24 \mu \mathrm{s}$, which is close to $\operatorname{NOX}(23 \mu \mathrm{s})$ and Floodlight $(21 \mu \mathrm{s})$ latency values. With one switch, OpenDaylight's 
flow setup latency is $108 \mu \mathrm{s}$, which is high compared to other controllers. With 8 switches and beyond, ONOS outperforms other controllers in the latency test. It shows $3 \mu$ s to 4 $\mu$ s of flow setup latency. With 16 switches and more, OpenDaylight's flow setup latency is better than most controllers, except ONOS and OpenIRIS.

Figures 4.9 and 4.10 illustrate the ONOS and OpenDaylight average flow setup latency with different numbers of hosts. For ONOS, the vertical axis scale ranges from 0 to 12 . For OpenDaylight, the vertical axis scale ranges from 0 to 25 . The error bars are barely visible in both graphs.

ONOS and OpenDaylight have similar flow setup latency trends in this test. ONOS's average flow setup latency is around $4 \mu$ s on the physical host, regardless of the number of hosts. On the physical host without HT, ONOS shows almost the same performance. On the virtual host, the latency is around $10 \mu \mathrm{s}$. This is about double ONOS's latency on the physical host.

As shown in Figure 4.10, OpenDaylight shows higher flow setup latency compared to ONOS. OpenDaylight's flow setup latency is around $10 \mu \mathrm{s}$ on the physical host. The performance with and without HT is similar in this test.

For OpenDaylight, on the virtual host, the latency is around $20 \mu \mathrm{s}$, regardless of the number of hosts. The latency on the virtual host is about double its latency on the physical host. Similar to the throughput test, these results show that the number of hosts in the network has low impact on the average flow setup latency. 


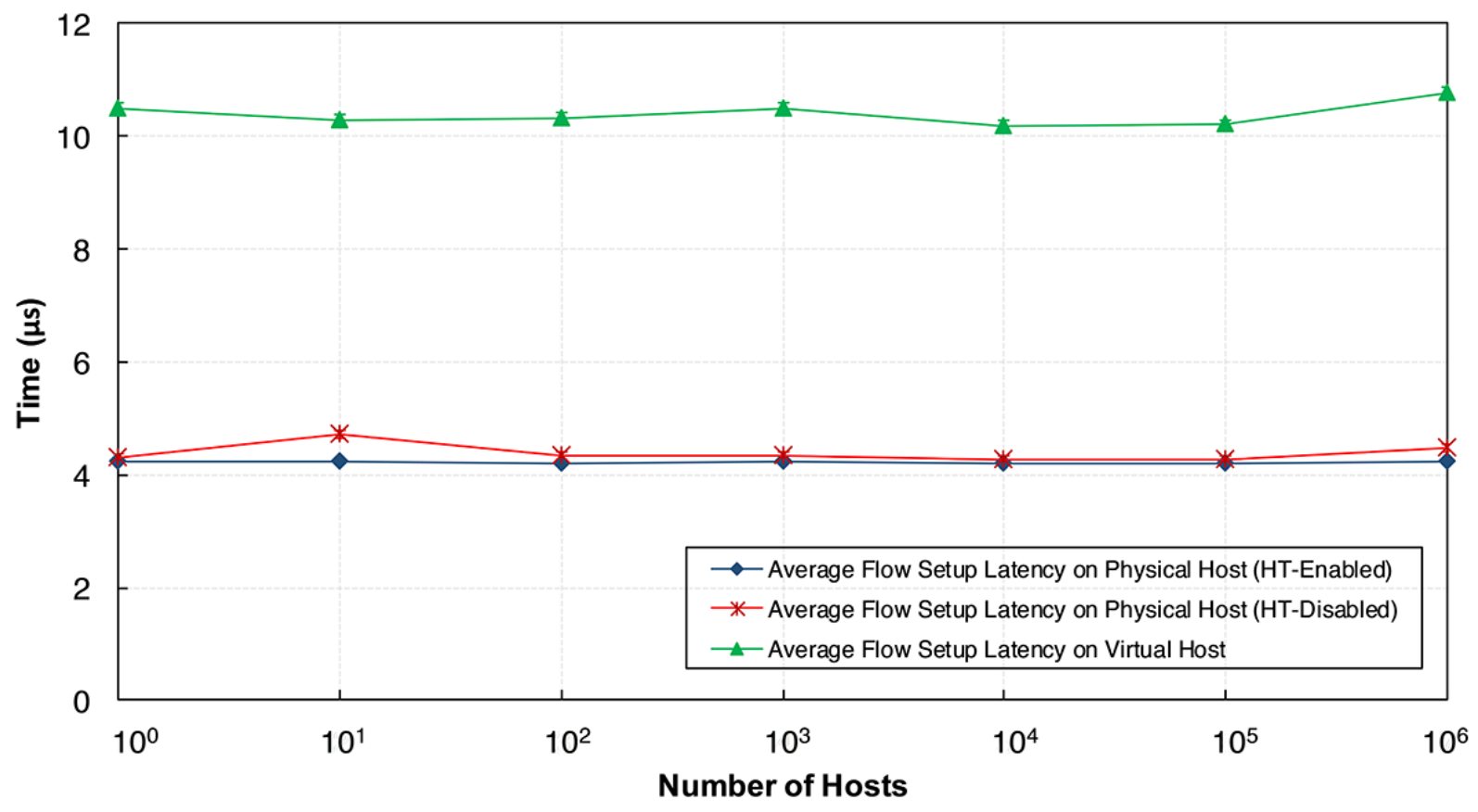

Figure 4.9: ONOS average flow setup latency with different number of hosts

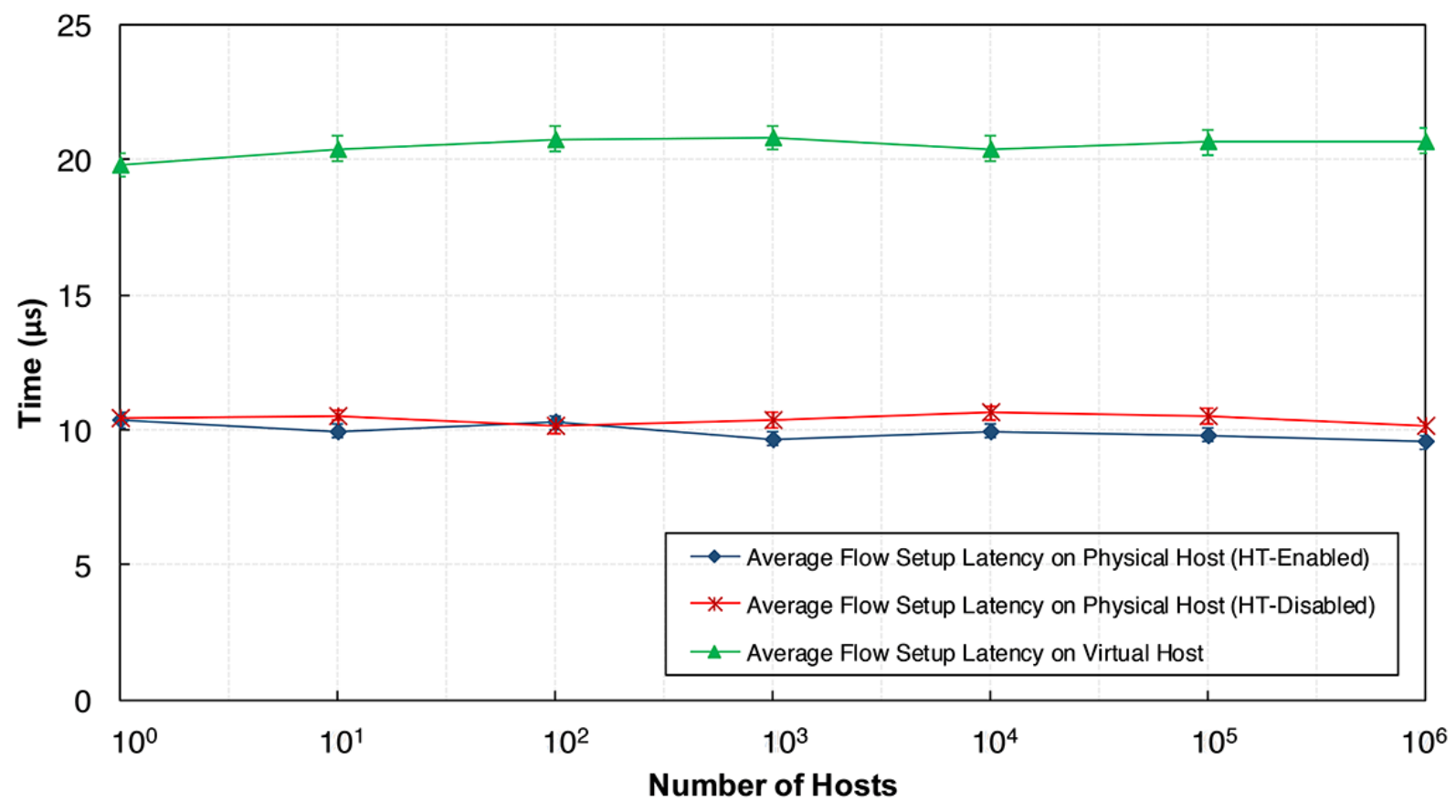

Figure 4.10: OpenDaylight average flow setup latency with different number of hosts 


\subsection{Thread Scalability Results}

In this section, we present the results of our CPU thread scalability tests on ONOS and OpenDaylight. In both experiments, we used a different number of threads to identify the effects on throughput and latency. We kept the number of hosts and switches fixed to identify the impact of adding more CPU threads to the controllers. We conducted the tests with 16 emulated switches, based on the outcome of throughput tests (Figure 4.1 and Figure 4.2).

For the thread scalability tests, we examined throughput and latency with 1 to 16 threads. We limited the number of threads to 16 for consistency in all experiments. Without HT, the server used to run the controllers has only 24 threads, some of which are used for running the operating system. For experiments on the virtual host, we reserved 8 threads on the server to run the host operating system, OpenStack, and its services.

Figures 4.11 and 4.12 illustrate the average throughput of ONOS and OpenDaylight with different numbers of threads. Both controllers exhibit a similar trend in this test. Throughput increases steadily at first, with a plateau at 12-16 threads, which reveals their multithreading capabilities. Unlike the majority of SDN controllers, ONOS and OpenDaylight are not limited to 8 threads [56] [72] [83], and perform well across all 16 threads.

As shown in Figure 4.11, ONOS's throughput on the physical host with and without HT is similar. ONOS's average throughput on the physical host with one thread is $190 \mathrm{~K}$ (resp/s). The average throughput with 2 threads is $290 \mathrm{~K}(\mathrm{resp} / \mathrm{s})$ and reaches $640 \mathrm{~K}(\mathrm{resp} / \mathrm{s})$ with 5 threads. With 16 threads, ONOS's throughput reaches $1.47 \mathrm{M}$ (resp/s).

On the physical host without HT, the throughput is slightly better with 6-10 threads. ONOS's average throughput with 6-10 threads are 785K (resp/s), 918K (resp/s), 1.04M (resp/s), and 1.11M (resp/s), respectively. The standard deviations for these data points are around 30K (resp/s) which is larger than the mentioned performance gap. Similar to the throughput results with HT, there is a decline in throughput with 12-15 threads.

ONOS's average throughput on the virtual host, with one thread, is about $70 \mathrm{~K}$ (resp/s). 
The throughput with 16 threads is $1.21 \mathrm{M}(\mathrm{resp} / \mathrm{s})$, which is about $260 \mathrm{~K}(\mathrm{resp} / \mathrm{s})$ lower than the throughput on the physical host. Unlike the results on the physical host, there is no decline in throughput with 12-15 threads on the virtual host.

As mentioned above, we observed that the ONOS throughput decreased slightly after 12 threads (between 12-15 threads) and rose again with 16 threads. This trend reflects the communication delay between threads on separate sockets. As explained in Chapter 3, the server used to run the controllers has 48 threads (with HT), and 2 CPU sockets. Each processor socket has 24 threads. Threads $0-11$ and 24-35 reside on socket 1 , and threads 12-23 and 36-47 reside on socket 2 . We used the isolcpus and taskset commands to isolate and bind the desired number of threads to controllers. We isolated and pinned the first 16 threads to each controller at run time. Hence, the first 12 threads (thread 0-11) reside on processor socket 1 , and threads $12-15$ reside on processor socket 2 . Cores (threads) on the same socket typically share L2 and L3 caches [4], whereas cores on different sockets usually communicate via memory or use cache coherency protocol (cache-to-cache communications). The additional overhead for this coordination affects the achievable throughput.

The average throughput on the virtual host doesn't show the downturn between 1216 threads since the CPU allocation to the virtual machine (pinning guest vCPUs to host pCPUs) is automatically handled by the libvirt driver in OpenStack Nova. Since the guest operating system (virtual machine) only sees one processor socket, all 16 threads are on the same socket. Hence, further increasing the number of threads could result in better performance on the virtual host compared to the physical machine. A related point to consider is that in the OpenStack configuration, we set the CPU overcommit ratio to 1 , which means one vCPU of each VM corresponds to one pCPU on the bare-metal host.

Figure 4.12 shows the corresponding results for OpenDaylight. The graph uses the same format as Figure 4.11, except that the vertical axis scale ranges from 0 to $260 \mathrm{~K}$, instead of 0 to $1.6 \mathrm{M}$. 


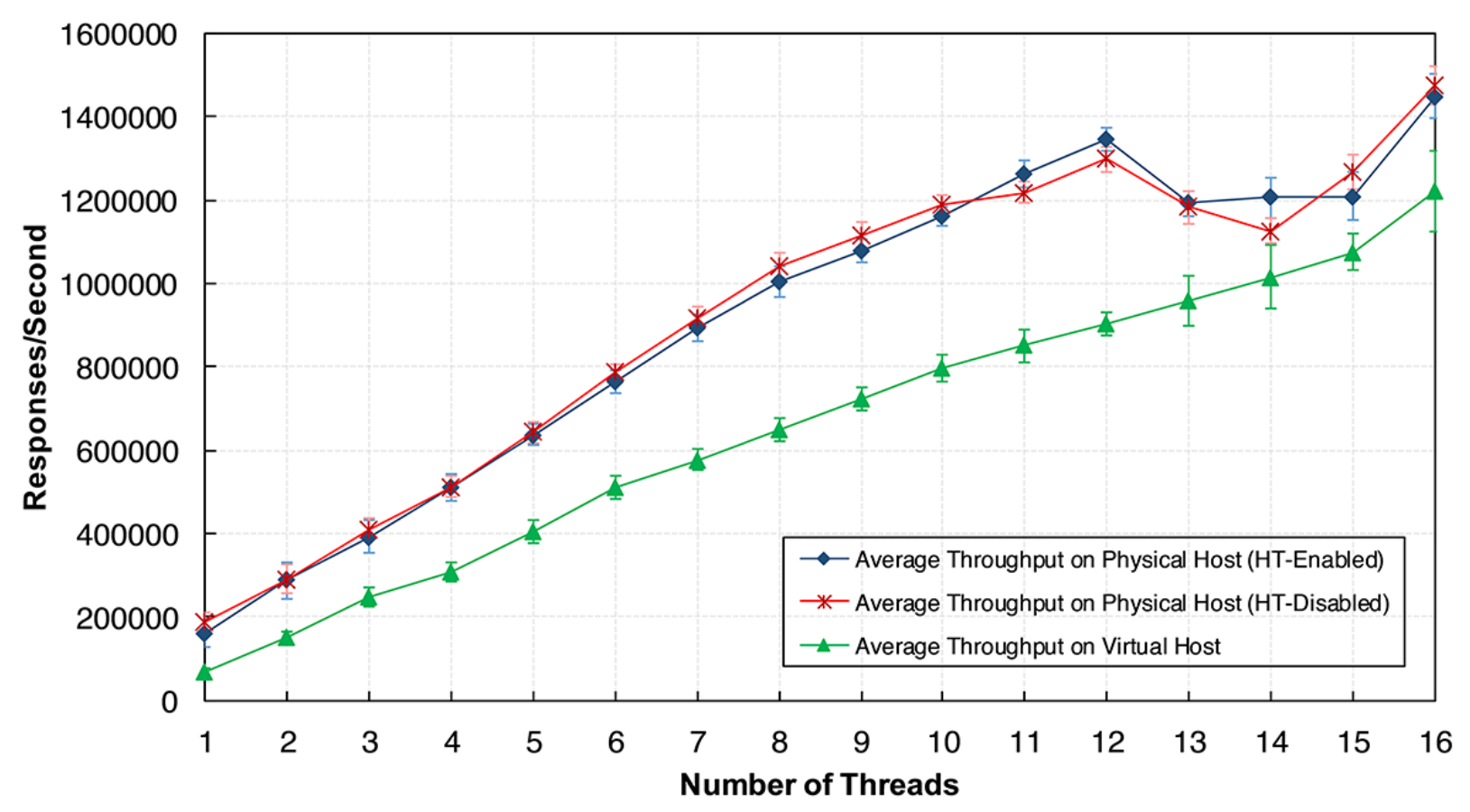

Figure 4.11: ONOS average throughput with different number of threads

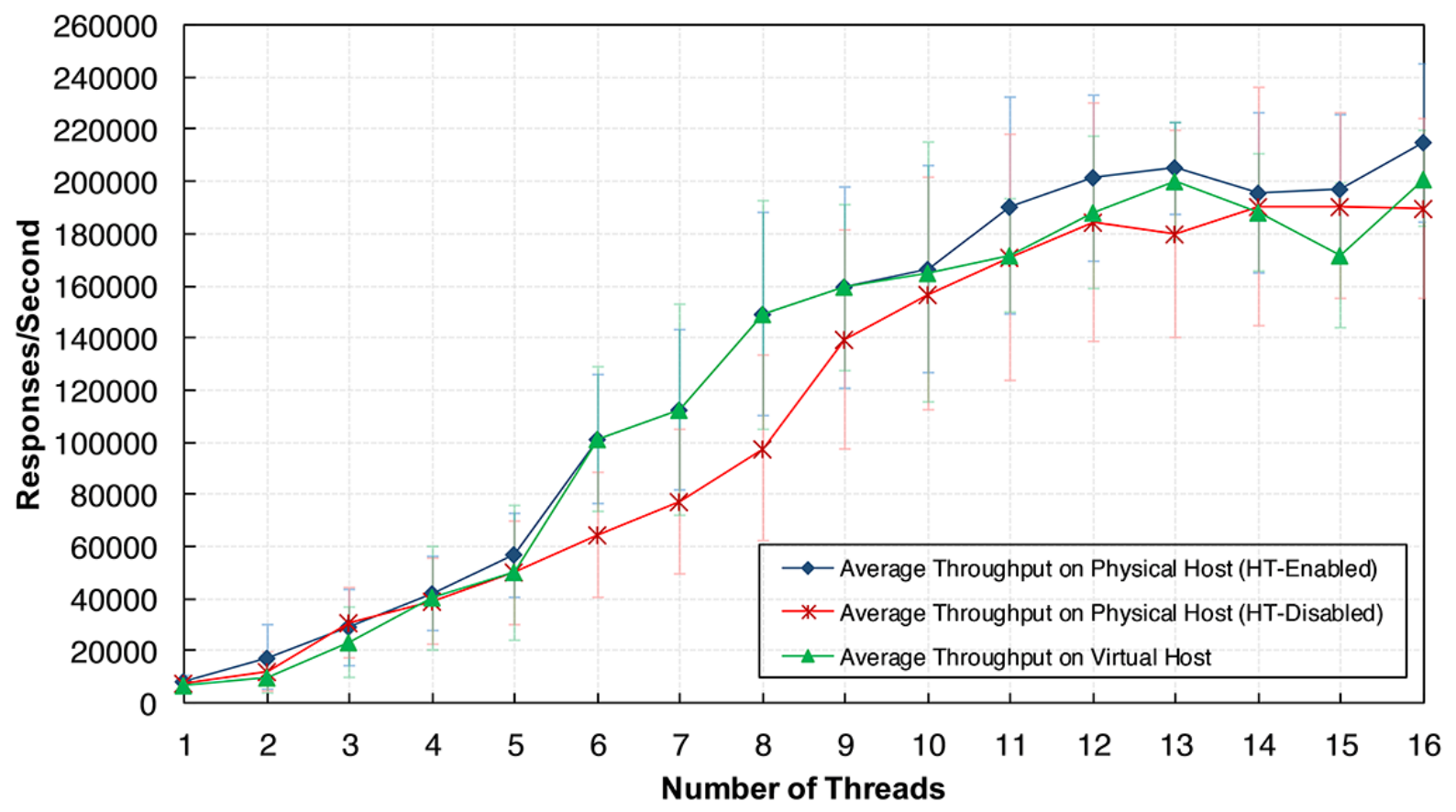

Figure 4.12: OpenDaylight average throughput with different number of threads 
As shown in Figure 4.12, OpenDaylight delivers an average throughput of $9 \mathrm{~K}$ (resp/s) with one thread on the physical host with HT. The throughput increases by adding more threads. For instance, the throughput with 5 threads reaches $60 \mathrm{~K}$ (resp/s). The throughput improvements observed between threads 5-6 and threads 7-8 are higher compared to the throughput increase between other threads. The decline in throughput between 12-15 threads happens, but with minor effect. OpenDaylight's throughput with 16 threads is $215 \mathrm{~K}$ (resp/s).

On the physical host without HT, OpenDaylight shows its lowest throughput. Even though increasing the number of threads results in higher throughput, between 6-10 threads the performance is considerably lower than the results with HT. There is a slight decline in throughput between 12-13 threads. The throughput with 16 threads is about $190 \mathrm{~K}$ (resp/s).

OpenDaylight's throughput on the virtual host, with 1-10 threads, is similar to its throughput on the physical host with HT. The decline in throughput occurs between 1315 threads. The throughput with 16 threads is $200 \mathrm{~K}$ (resp/s).

As shown in Figure 4.12, OpenDaylight's responses in this test show large standard deviations. First of all, it should be pointed out that in all throughput tests, OpenDaylight showed greater standard deviation in its responses compared to ONOS. However, in this experiment, we observed relatively larger standard deviations, especially when we run OpenDaylight with fewer than 16 threads. For example, the throughput with 16 threads on the physical host with $\mathrm{HT}$ is $215 \mathrm{~K}(\mathrm{resp} / \mathrm{s})$. The standard deviation at this point is $30 \mathrm{~K}(\mathrm{resp} / \mathrm{s})$. With 8 threads, the throughput is $148 \mathrm{~K}$ (resp/s), and the standard deviation is $35 \mathrm{~K}$ (resp/s). We speculate that this may reflect an issue in OpenDaylight's OpenFlow plugin, which fails to process packet_in messages in a consistent manner.

Recent studies on the performance of SDN controllers indicate that among all well-known multi-threaded controllers, only Beacon, IRIS, ONOS, and OpenDaylight are able to utilize more than 8 threads effectively 72 , 83 .

We compared the throughput of the single thread ONOS in our study with prior work 99$]$. 
Table 4.13 compares the average throughput of two different releases of ONOS with one connected switch. The performance difference in Table 4.13 indicates substantial throughput improvement of Goldeneye release compared to Blackbird release.

Table 4.13: ONOS throughput comparison with prior work $[99$

\begin{tabular}{|c|c|c|c|}
\hline \# Switch & \# Thread & Avg. TP with Blackbird |99| & Avg. TP with Goldeneye \\
\hline 1 & 1 & $49 \mathrm{~K}(\mathrm{resp} / \mathrm{s})$ & 190K (resp/s) \\
\hline
\end{tabular}

The authors of [99] also evaluated the throughput of OpenDaylight with one thread. Table 4.14 presents the average throughput of two different releases of OpenDaylight with one connected switch.

Table 4.14: OpenDaylight throughput comparison with prior work $\mid 99$

\begin{tabular}{|c|c|c|c|c|}
\hline \# Switch & \# Thread & Avg. TP with Helium-SR3 & 99 & Avg. TP with Beryllium-SR2 \\
\hline 1 & 1 & $8 \mathrm{~K}(\mathrm{resp} / \mathrm{s})$ & $9 \mathrm{~K}(\mathrm{resp} / \mathrm{s})$ \\
\hline
\end{tabular}

Figures 4.13 and 4.14 portray the latency test results with different numbers of threads. In both graphs, the vertical axes indicate the average flow setup latency, in microseconds. For ONOS, in Figure 4.13, the vertical axis ranges from 0 to 35. For OpenDaylight, in Figure 4.14, the vertical axis scale ranges from 0 to 160 .

Both controllers demonstrate structurally similar trends in this test. In Figure 4.13, ONOS's average flow setup latency with 1 thread is around $15 \mu$ s on the physical host with HT. ONOS's average flow setup latency with 2,3 , and 4 threads are $8.5 \mu \mathrm{s}, 5.5 \mu \mathrm{s}$, and $4.5 \mu \mathrm{s}$, respectively. With 4 threads (and above), ONOS shows its lowest latency, which is around $4 \mu \mathrm{s}$.

ONOS's performance on the physical host without HT is similar. The average flow setup latency with 5 threads is slightly higher without $\mathrm{HT}$, and is about $4.7 \mu \mathrm{s}$. 


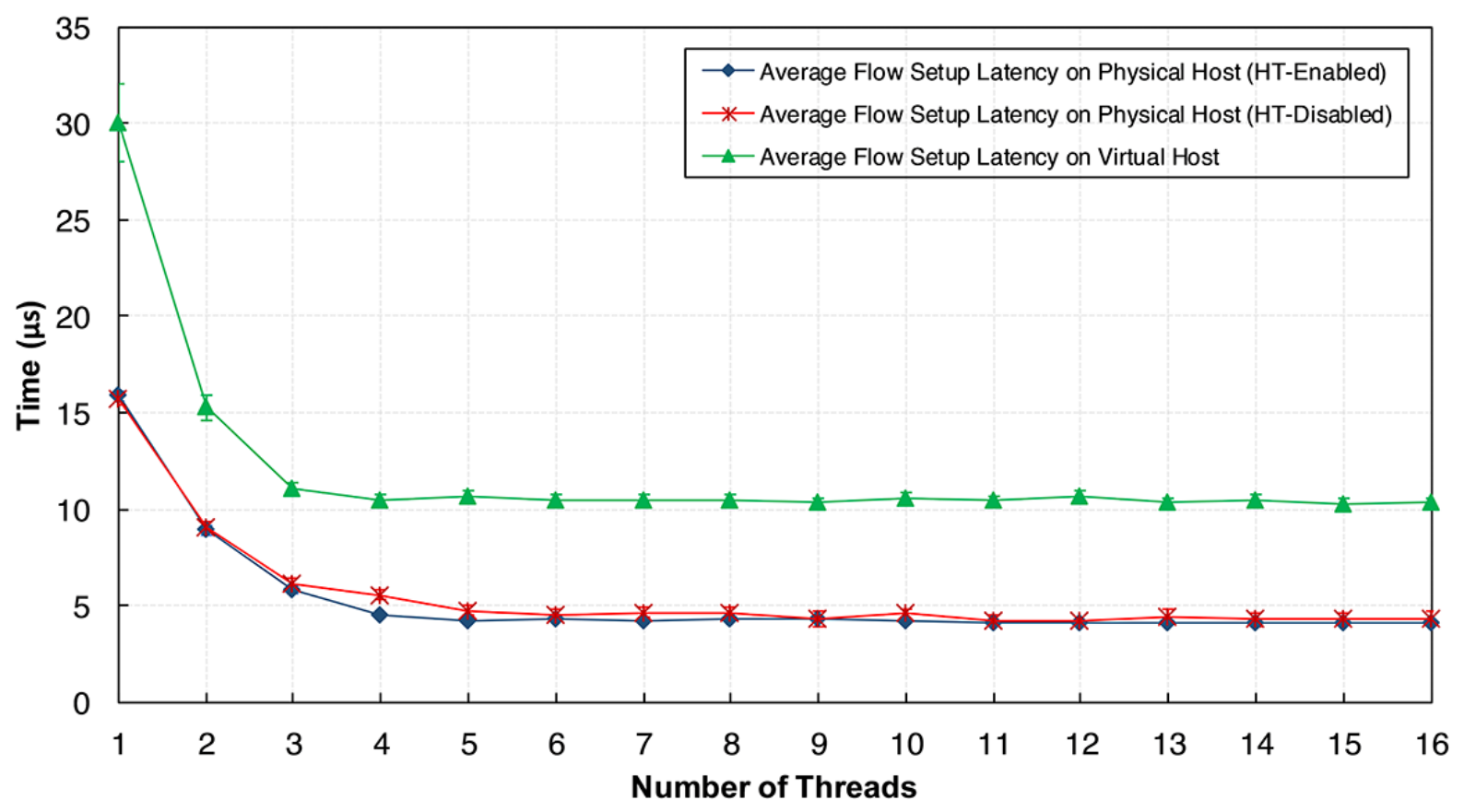

Figure 4.13: ONOS average flow setup latency with different number of threads

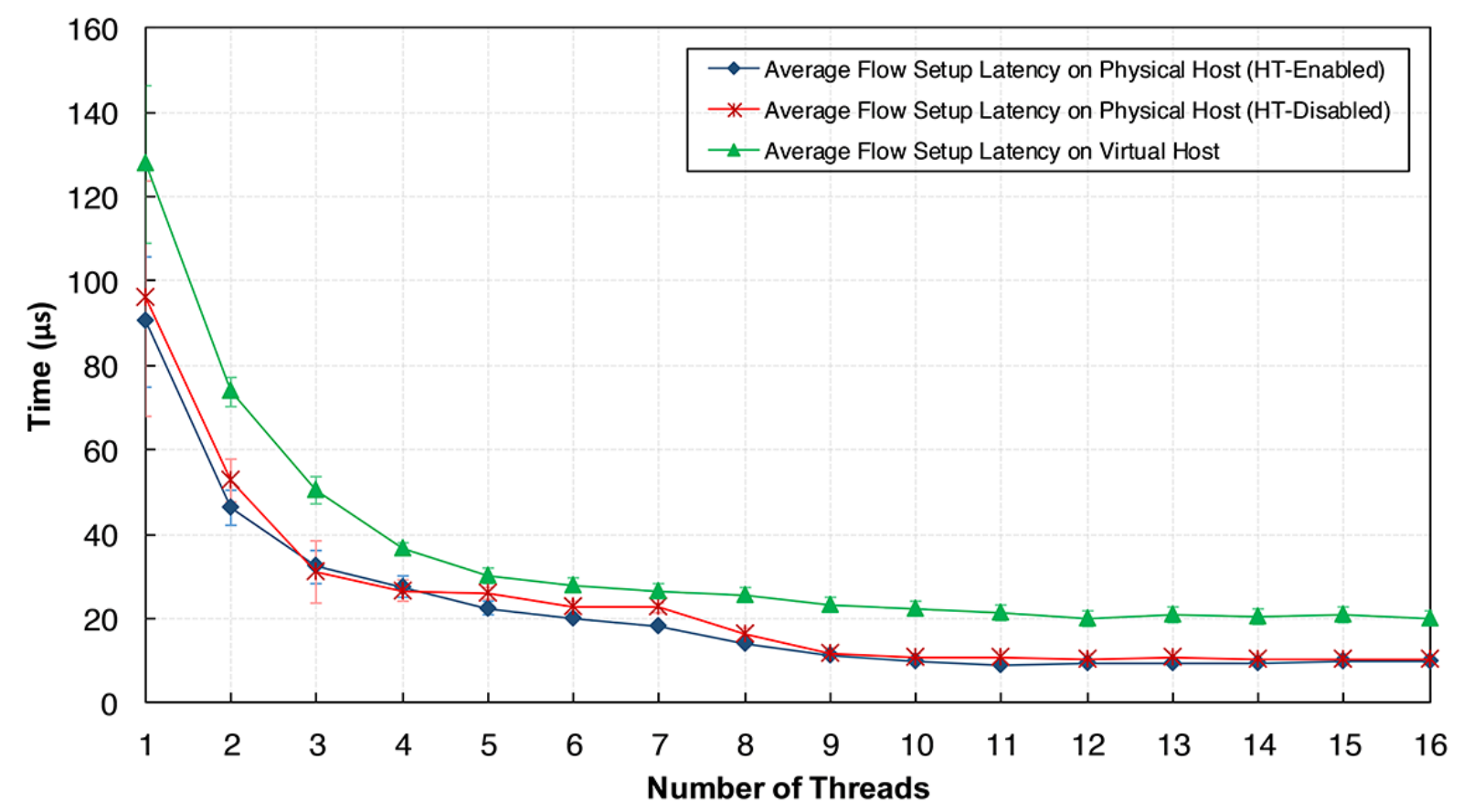

Figure 4.14: OpenDaylight average flow setup latency with different number of threads 
On the virtual host, ONOS's average flow setup latency is $29 \mu \mathrm{s}$. With 2-4 threads, ONOS latency values are $15 \mu \mathrm{s}, 11 \mu \mathrm{s}$, and $10.5 \mu \mathrm{s}$. Adding more than 4 threads doesn't decrease the latency of ONOS. The average flow setup latency with more than 4 threads is $10.5 \mu \mathrm{s}$.

For ONOS, the error bars for the physical host are barely visible. On the virtual host, the standard deviations become very small when increasing the number of threads, and are not visible beyond 4 threads.

In Figure 4.14, OpenDaylight's average flow setup latency with 1 thread is around $90 \mu \mathrm{s}$ on the physical host with HT. OpenDaylight's average flow setup latency with 2, 3, and 4 threads are $46 \mu \mathrm{s}, 32 \mu \mathrm{s}$, and $27 \mu \mathrm{s}$, respectively. OpenDaylight reaches its lowest latency with the use of 10 threads, where it has $10 \mu$ s of average flow setup latency.

Without HT, the results are similar for OpenDaylight. The average flow setup latency with 1 thread is $95 \mu \mathrm{s}$. With 2 threads, the latency drops to $52 \mu \mathrm{s}$. With $3-4$ threads, OpenDaylight's latency is similar to results with HT. With 5-9 threads, the average flow setup latency is slightly higher than with HT. OpenDaylight with 10 threads (and more) shows its lowest flow setup latency on the physical host without HT, which is $10 \mu \mathrm{s}$.

On the virtual host, OpenDaylight's average flow setup latency is higher than the physical host results. With the use of one thread, the latency is around $127 \mu$ s. Adding more threads leads to lower latency results. Similar to the physical host, OpenDaylight shows its lowest latency with the use of 10 threads. The latency with 10 threads (and more) is $20 \mu \mathrm{s}$, which is double the latency values on the physical host.

Both controllers are able to reduce flow setup latency using more threads. For both controllers, the latency on the virtual host is about double that on the physical host, when sufficient threads are used.

We compared the latency of the single thread ONOS and OpenDaylight in our study with prior work [99]. Table 4.13 shows the average flow setup latency of ONOS Blackbird 
release in the previous study, and compares it with Goldeneye release in our study. On the other hand, Table 4.16 presents the average flow setup latency of OpenDaylight Helium-SR3 in prior work, and compares it with the Beryllium-SR2 release in our study. The presented flow setup latencies in the following tables are with one connected switch.

Table 4.15: ONOS latency comparison with prior work 99

\begin{tabular}{|c|c|c|c|}
\hline \# Switch & \# Thread & Avg. LT with Blackbird |99| & Avg. LT with Goldeneye \\
\hline 1 & 1 & $26 \mu \mathrm{s}$ & $24 \mu \mathrm{s}$ \\
\hline
\end{tabular}

Table 4.16: OpenDaylight latency comparison with prior work 99$]$

\begin{tabular}{|c|c|c|c|}
\hline \# Switch & \# Thread & Avg. LT with Helium-SR3 |99| & Avg. LT with Beryllium-SR2 \\
\hline 1 & 1 & $111 \mu \mathrm{s}$ & $108 \mu \mathrm{s}$ \\
\hline
\end{tabular}

\subsection{Summary}

In this chapter, we presented the results of our Cbench experimental evaluation of ONOS and OpenDaylight. In the throughput test, ONOS showed better and more robust performance than OpenDaylight. With ONOS, the highest average throughput achieved was 1.5 million responses/sec for 64 switches. OpenDaylight showed its highest throughput of around $270 \mathrm{~K}$ responses/second with 8 switches. The throughput of ONOS with and without HyperThreading was almost the same, and the average throughput on the virtual host was about $13.5 \%$ lower. On the other hand, OpenDaylight showed drastic throughput degradation with more than 8 switches. OpenDaylight showed its worst performance on the physical host when Hyper-Threading is turned off. In the next chapter, we perform a few follow-up tests to investigate the OpenDaylight throughput issue. 


\section{Chapter 5}

\section{Additional Experimental Results}

In this chapter, we present and discuss the results of our additional experiments on ONOS and OpenDaylight. In particular, in Section 5.1, we show the results of memory profiling of controllers under the throughput test. In Section 5.2, we study CPU utilization of both controllers in throughput tests, which helps to better understand the cause of OpenDaylight's poor performance. Finally, we discuss the results of our follow-up tests on thread scalability of controllers when threads reside on one socket.

\subsection{Memory Usage Results}

In the discussion section of [60], the authors speculated that OpenDaylight might have a memory leak, which could be the reason for its poor performance in the throughput test. We investigated this issue further, and managed to show that OpenDaylight's memory management is normal, and it doesn't have a memory leak.

A memory leak in a Java application happens when objects that are no longer needed are superfluously maintained in memory [96]. In other words, the memory leak in Java is a situation where the created objects, which are being stored in the Heap memory, are not used by the application anymore. However, the garbage collector fails to recognize them as unused. Hence, they still remain in the Heap memory.

A memory leak can put unnecessary stress on the memory of the machine, and exhaust the resources. The Java Virtual Machine (JVM) is responsible for scheduling and execution of garbage collection (GC) to reclaim memory (created Java objects that are never explicitly

deallocated [98]). Garbage collection is an automatic memory management approach in modern programming languages, such as Java. 
Java uses a generational garbage collector [59], which relies on two assumptions. First, most of the created objects are quickly discarded. Second, there are not many references from old objects to young objects. Figure 5.1 exhibits the generations of the garbage collection. JVM Heap memory is physically divided into two spaces (referred to as generations), namely young generation, and old generation. There is also a third space in the Java's memory layout (not part of the Heap) known as permanent generation [55] [70].

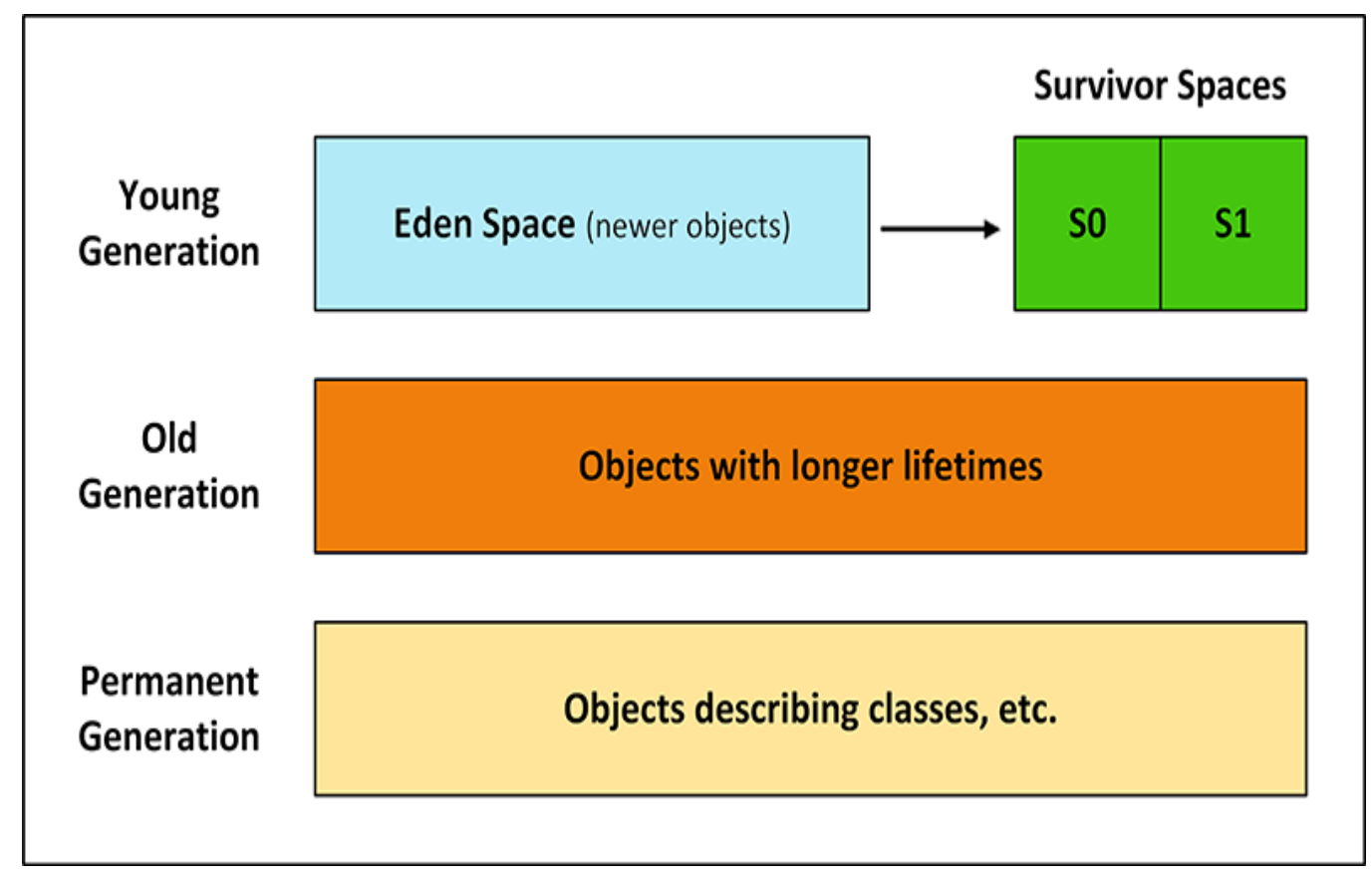

Figure 5.1: Garbage collection generations

The young generation is where objects first appear. Most of the newly generated objects are placed in this space. The young generation has two sub-generations, namely Eden space and Survivor space. Eden space is where most of the objects are generated and then destroyed with a minor garbage collection. In other words, after a minor GC in Eden space, the references to objects that are still in use need to migrate to Survivor space. That being said, the objects that survive from Eden space end up in the Survivor space.

There are two survivor spaces, namely S0 and S1. Only one of these spaces is in use at a given time, and the other one is empty. Otherwise, it indicates that the application has a 
memory leak.

The next space in the Heap memory is the old generation. It is also known as the tenured generation, and is typically larger than the young generation. It is a space for objects with longer lifetimes. After minor GC(s) in the young generation, the objects from Survivor space that are still in use move to this area.

The third area in Java's memory pool for garbage collection is the permanent generation. Even though it is categorized as a generation, the objects do not transfer from old generation to this area. Instead, it is used by JVM to keep class metadata (e.g., class data structures, strings, etc.).

The JVM garbage collector performs two operations to reclaim unused memory, namely minor garbage collection and full garbage collection.

Figure 5.2 shows the Heap memory before and after minor GC (called young collection) [70]. Minor GC occurs when the Eden space is full. At the end of the minor GC, all existing objects in the Eden space transfer out. Live objects transfer to the empty Survivor space (S0 in our diagram), and objects that are already in the other Survivor space (S1) move to the old generation. It is worth noting that many objects are discarded with minor GC since they are no longer in use. Minor GC is usually quite fast, and does not impact the performance of the application.

On the other hand, Figure 5.3 illustrates the schematic view of Heap memory before and after full GC [70]. Full GC occurs when the Heap memory is full. Full GC empties the young generation (including Eden space and Survivor spaces) and compacts old generation objects. The objects that remain in the old generation after full GC are those that have active references. Major GC takes longer compared to minor GC since it needs to scan all of the Heap memory for live objects. The frequency of full GC should be minimized since it may negatively impact the performance of the application. 
Before minor GC

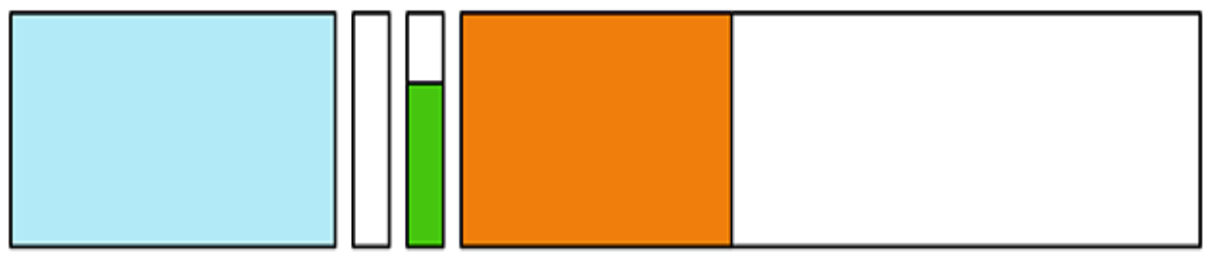

Eden

So S1 Old Generation

After minor GC

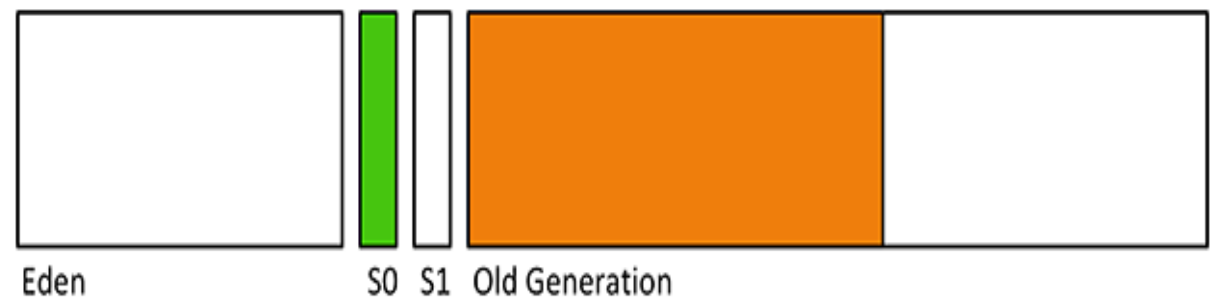

Figure 5.2: Schematic view of Heap memory before and after minor GC

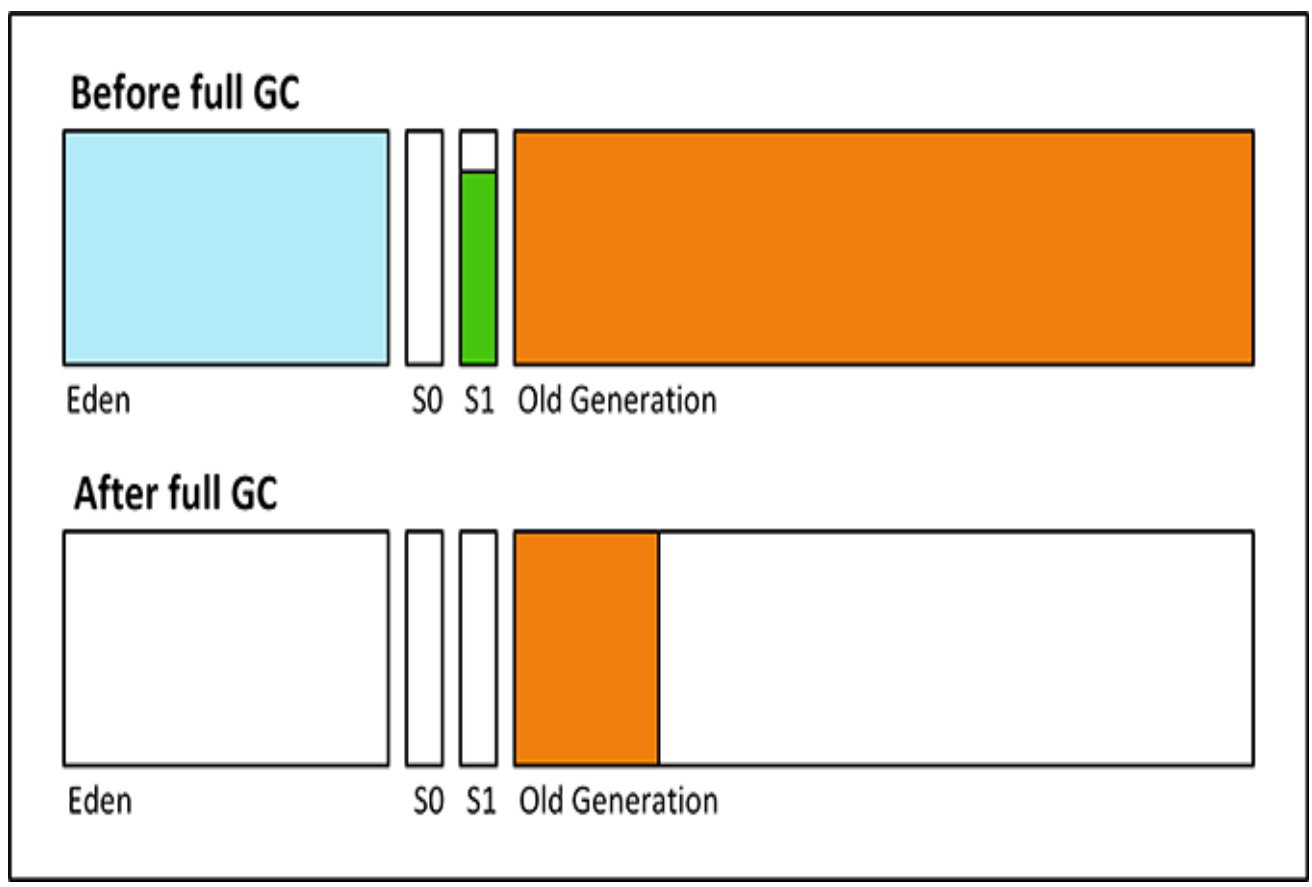

Figure 5.3: Schematic view of Heap memory before and after full GC 
Making the young generation larger results in fewer minor GCs. However, this means that the size of the old generation becomes smaller, which increases the frequency of full GCs.

By default, the ratio between the young and old generation is 1:2. It means the JVM allocates $1 / 3$ of Heap memory to the young generation (Eden and the Survivor spaces), and the rest (2/3 of the Heap) is occupied by the old generation [19]. However, the JVM allows us to control the Heap size, and adjust the default ratio to change the size of young and old generation.

We used YourKit 28] (version 2016.02-b46), one of the most recognized profiling tools for CPU and memory profiling, to scrutinize OpenDaylight memory usage under test load. To capture memory usage statistics of controllers, we used a remote profiling approach. To this end, we downloaded the profiler's agent to the controller's server. Then we started the controller with the agent's path to load it into JVM. Subsequently, we executed the YourKit shell script on the server, and attached it to the PID of the running controller. As shown in Figure 5.4, upon successful completion of these steps, YourKit Profiler's agent generates a prompt on the terminal to indicate that it's ready to attach to the profiler UI. Lastly, we attached the profiler UI (installed on the remote machine) to the agent.

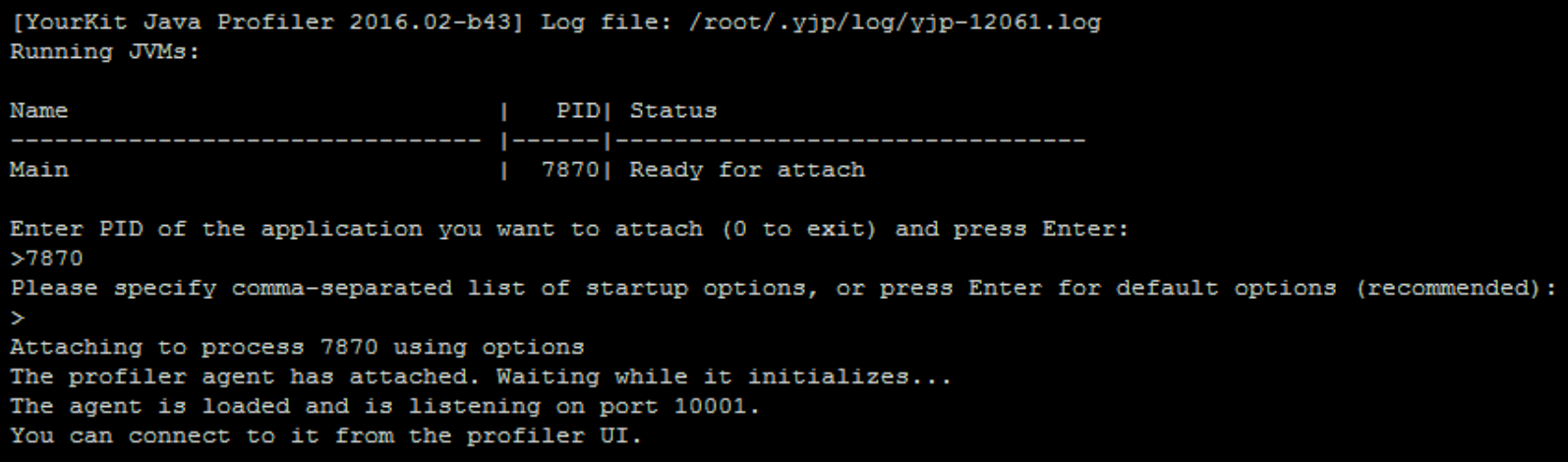

Figure 5.4: YourKit profiler's prompt when successfully attached to the controller

Figure 5.5a presents OpenDaylight's Heap memory usage before and during the through- 
put test. Figure $5.5 \mathrm{~b}$ shows Heap memory usage after test. Lastly, Figure $5.5 \mathrm{c}$ exhibits Heap memory utilization after full garbage collection. Heap memory is the storage of Java objects. In other words, Heap memory is the memory pool used by JVM for created objects in a Java application.

In Figures 5.5a, 5.5b, and 5.5c, the horizontal axis of each graph shows time in seconds. The vertical axis in each graph shows Heap memory usage. The vertical axes present the Heap memory usage in Gigabyte (GB). We conducted the memory profiling on a fresh install of OpenDaylight, and ran OpenDaylight with 16 threads for this test.

We assigned $16 \mathrm{~GB}$ of memory to the controller. Following the recommended Java settings for OpenDaylight, we set equal values for the minimum and maximum Heap size (16 GB).

The green area in each graph shows the entire size of young and old generations together. The blue area shows the Eden space usage. The orange and dark green lines at the very bottom of each graph indicate Survivor areas and old generation usage.

Based on the assigned memory, and recommended settings, the JVM allocates a small portion of memory to the permanent generation, which is considered Non-Heap memory. For OpenDaylight, the JVM assigned $200 \mathrm{MB}$ to the permanent generation. The size of Eden space, each Survivor space, and old generation are 5.1 GB, $250 \mathrm{MB}$, and $10.2 \mathrm{~GB}$, respectively.

In Figure 5.5a, when the test begins, the usage of Eden space is $361 \mathrm{MB}$. The Survivor spaces, and old generation usage, is 0 bytes.

The throughput test runs for about 125 seconds. In Figure 5.5a, the red arrow indicates the start of the test. The memory usage growth is normal as OpenDaylight allocates objects for its data. The spikes in the blue area show that the Eden space is almost full. The Eden space usage at most of the spikes is near 5.0 GB. As shown in Figures $5.5 \mathrm{a}$ and 5.5b, when the Eden space fills, a minor GC occurs. In Figure 5.5b, the red arrow indicates the end of the throughput test. After that point, the memory is still occupied, which is normal since 
the Eden space is filled partially, not completely. Hence, the minor GC is not triggered.

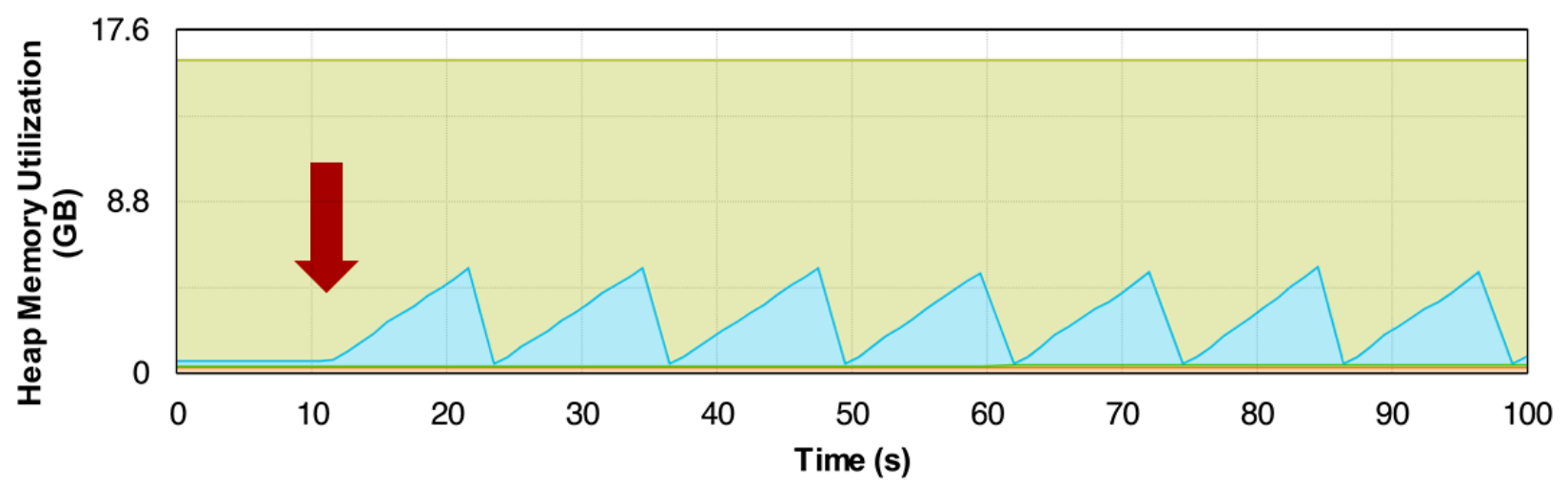

(a) OpenDaylight's memory usage (before and during test)

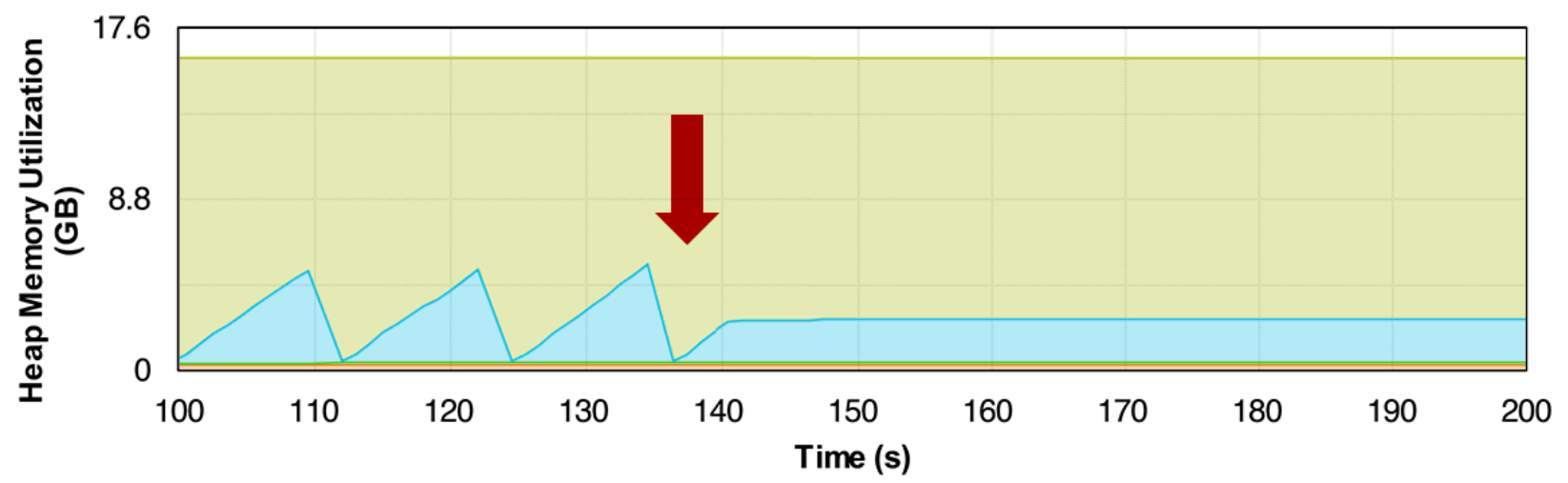

(b) OpenDaylight's memory usage (after test)

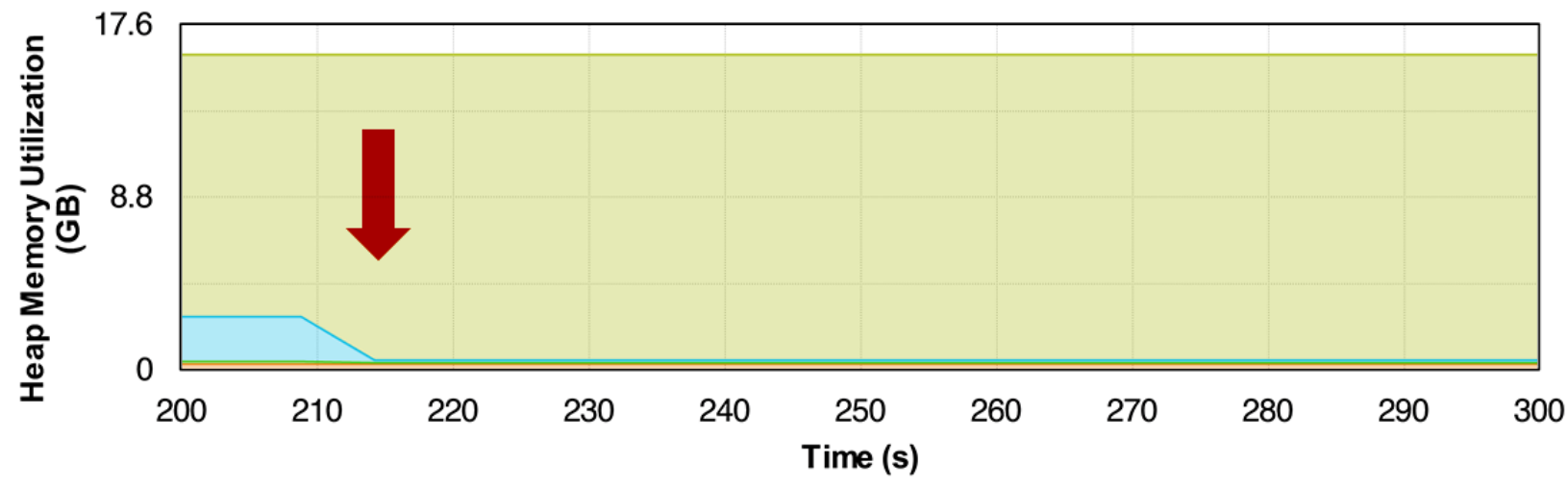

(c) OpenDaylight's Heap Memory usage (after full GC)

Figure 5.5: OpenDaylight Heap memory usage under throughput test 
In Figure 5.5c, we explicitly forced a full garbage collection (the red arrow) to reclaim the memory. After full GC, the Eden space and both Survivor spaces became empty. After 10 minor GCs during the throughput test, the usage of old generation is $180 \mathrm{MB}$, which is very low compared to its limit (10.2 GB). There is no evidence of a memory leak here. In programs with a memory leak, the old generation gets full quite fast, and even with a full $\mathrm{GC}$, the old generation is ever-growing [6].

We also used VisualVM [25] (version 1.3.9) with Visual GC plugin to study memory management of OpenDaylight. We used a different tool to repeat the test to ensure the accuracy of our results, and obtain better insight into OpenDaylight's GC activity. VisualVM is a powerful tool for monitoring Java application CPU usage, GC activity, etc. Visual GC is a plugin that integrates with VisualVM to graphically display garbage collection details.

We ran the second throughput test with the same parameters as the first test. Figure $5.6 a$ depicts generations of garbage collection before the start of the throughput test. Since OpenDaylight runs for the first time, there are no objects in Survivor spaces and old generation. As shown in Figure 5.6b, after the test, more than half of Eden space is full. During the test, 10 minor GCs occurred, which resulted in moving some objects into Survivor spaces, and then old generation.

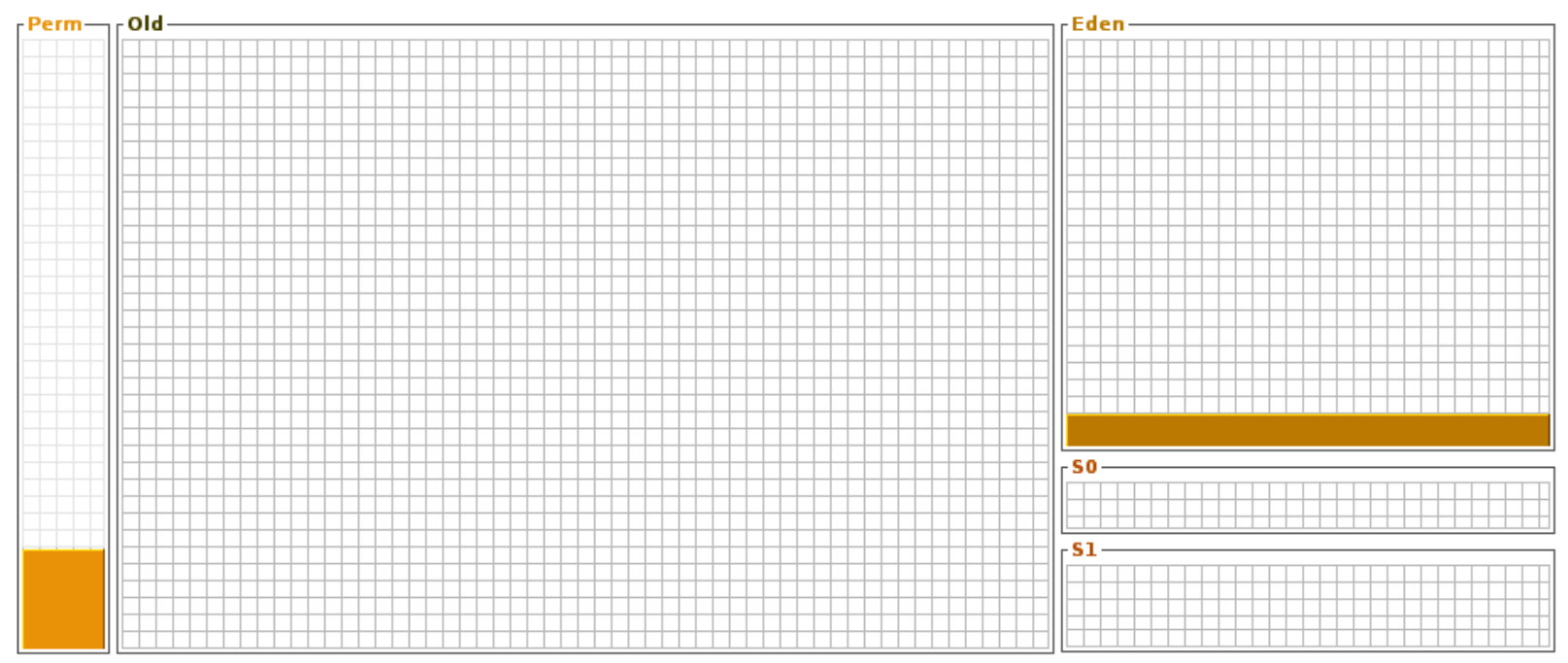

(a) GC generations before test 

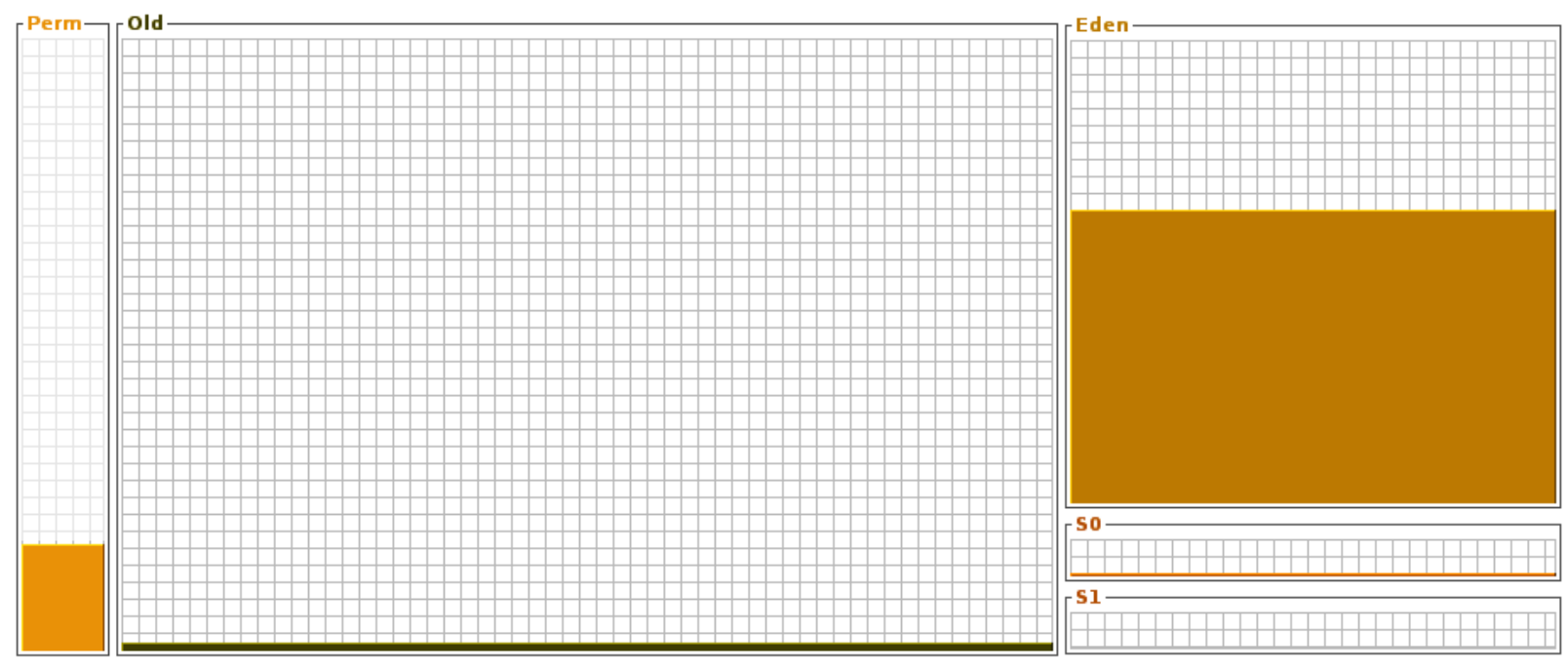

(b) GC generations after test

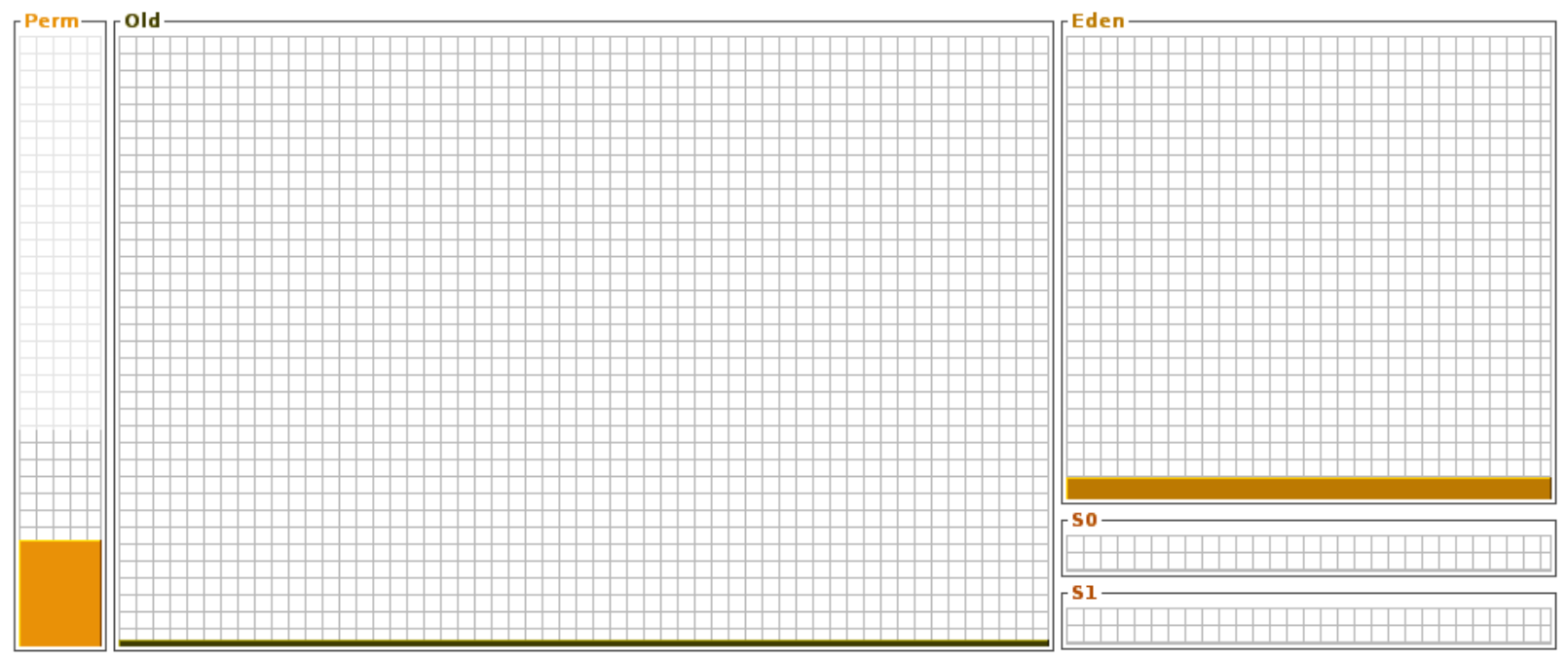

(c) GC generations after full GC

Figure 5.6: OpenDaylight GC generations in throughput test

In Figure 5.6b, S0 area shows usage of $55 \mathrm{MB}$, and the old generation is about $200 \mathrm{MB}$. As shown in Figure 5.6c, after performing a full GC, the size of old generation reduced to 158 MB. Also, both S0 and S1 spaces became empty. A related point to consider is that, even though the Eden space gets empty with minor or full GC, it is not supposed to remain empty afterward. In other words, depending on the application's usage, the Eden space usage may increase after garbage collection. 
For consistency, we also conducted memory profiling on ONOS to study its memory management. Similar to OpenDaylight, we conducted the throughput tests on a fresh install of ONOS. For the tests, we ran ONOS with 16 threads. We assigned 16 GB of memory to ONOS. The recommended Java settings for ONOS were the same as OpenDaylight. Hence, we set equal values (16 GB) for the minimum and maximum Heap size.

In Figures $5.7 \mathrm{a}, 5.7 \mathrm{~b}$, and $5.7 \mathrm{c}$, the horizontal axis of each graph shows time in seconds. The vertical axes show Heap memory utilization in Gigabyte (GB).

The red arrow in Figure 5.7a indicates the start of the test. Before that point, YourKit shows the Eden space usage is about $348 \mathrm{MB}$, and Survivor spaces and old generation usage is 0 bytes. As shown in Figure 5.7a, with start of the test, Eden space usage increases as ONOS creates objects. The spikes in the blue area show that the Eden space gets full, and subsequently, a minor GC occurs to free up the Eden area. The Eden space usage at most of the spikes is near $5 \mathrm{~GB}$.

ONOS's JVM defines the size of generations based on specified minimum and maximum Heap size, and its default setting. From the available memory pool, ONOS's JVM allocates 5.1 GB to Eden space, $500 \mathrm{MB}$ for both Survivor spaces, and 10.2 GB to the old generation. Also, it allocates $200 \mathrm{MB}$ to the permanent generation.

In Figure 5.7b, the red arrow indicates the end of the throughput test. At that point, the usage of old generation is $170 \mathrm{MB}$. After the test, the Eden space usage is around 2.6 GB, and the Eden space limit is 5.1 GB. Hence, it does not trigger the minor GC.

In Figure $5.7 \mathrm{c}$, as shown with the red arrow, we explicitly forced a full garbage collection to reclaim the memory. After full GC, the size of old generation reduced to $146 \mathrm{MB}$. Also, both Survivor spaces are empty. 


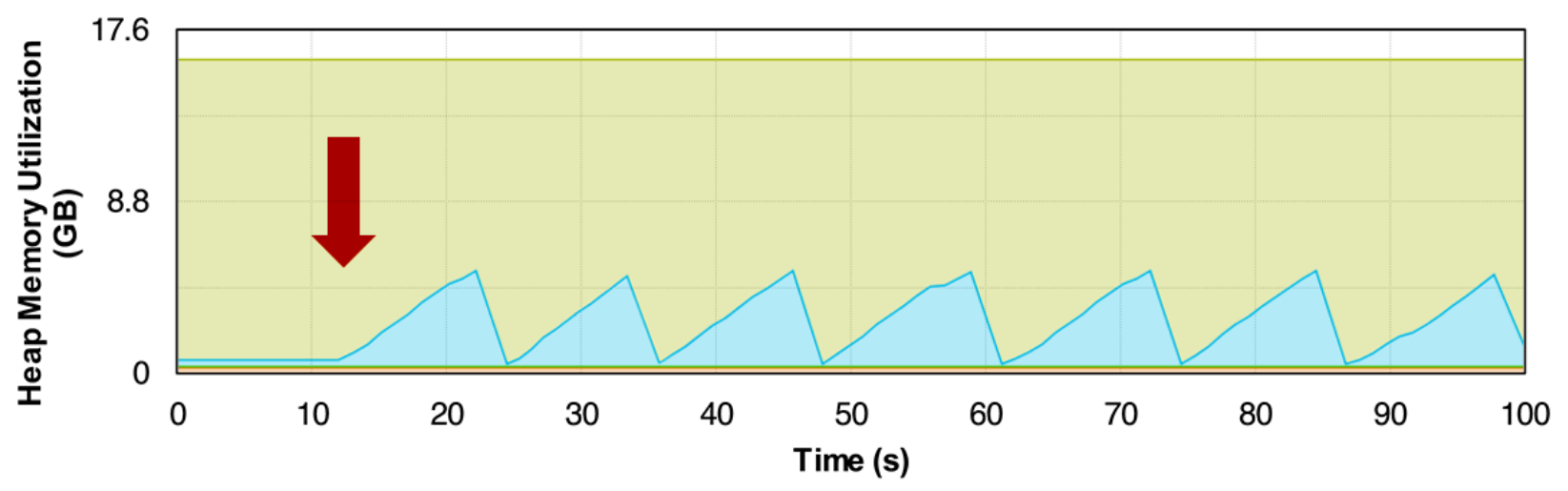

(a) ONOS's memory usage (before and during test)

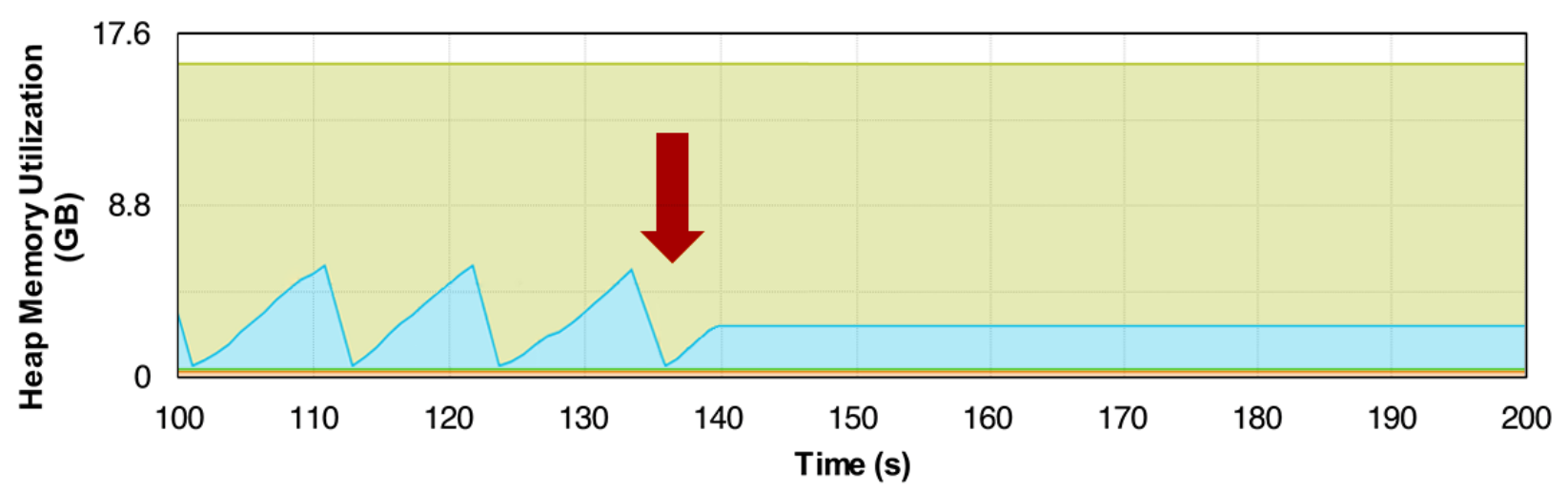

(b) ONOS's memory usage (after test)

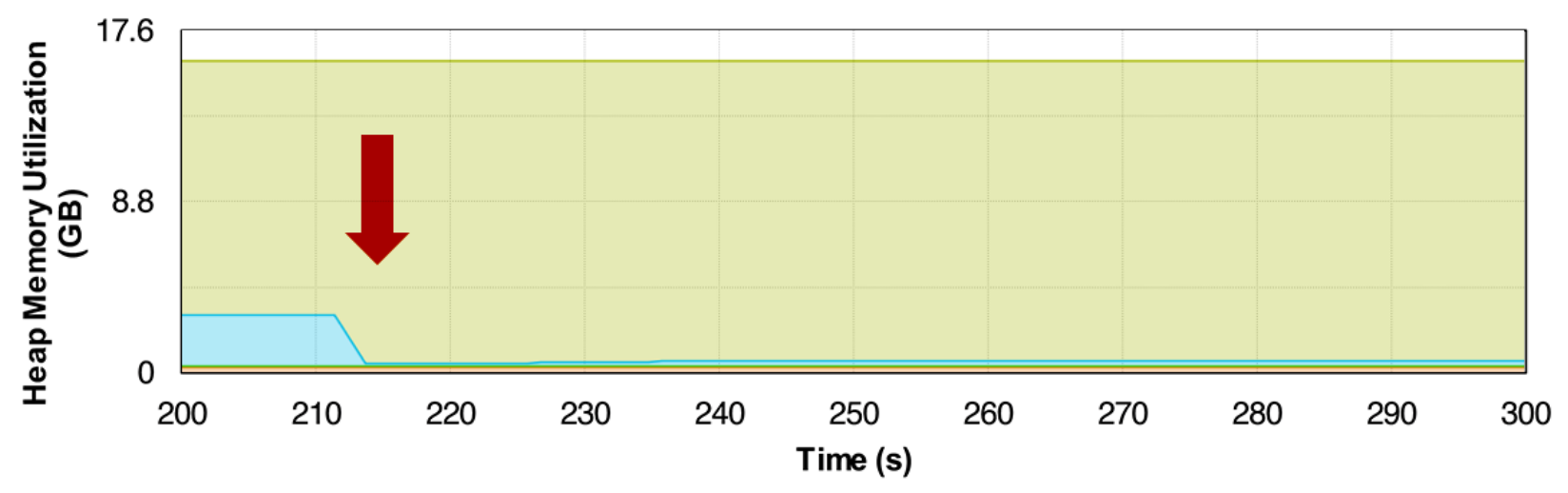

(c) ONOS's memory usage (after full GC)

Figure 5.7: ONOS's Heap memory usage under throughput test 


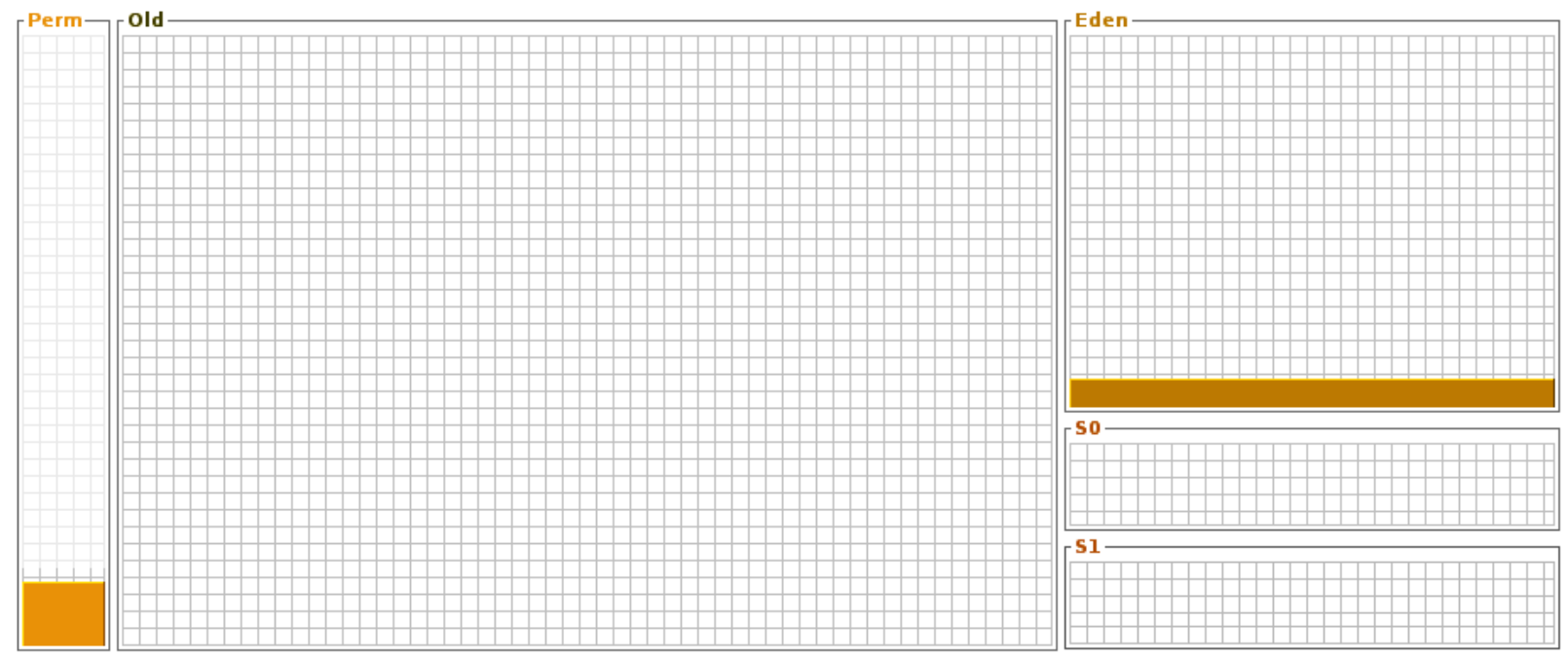

(a) GC generations before test

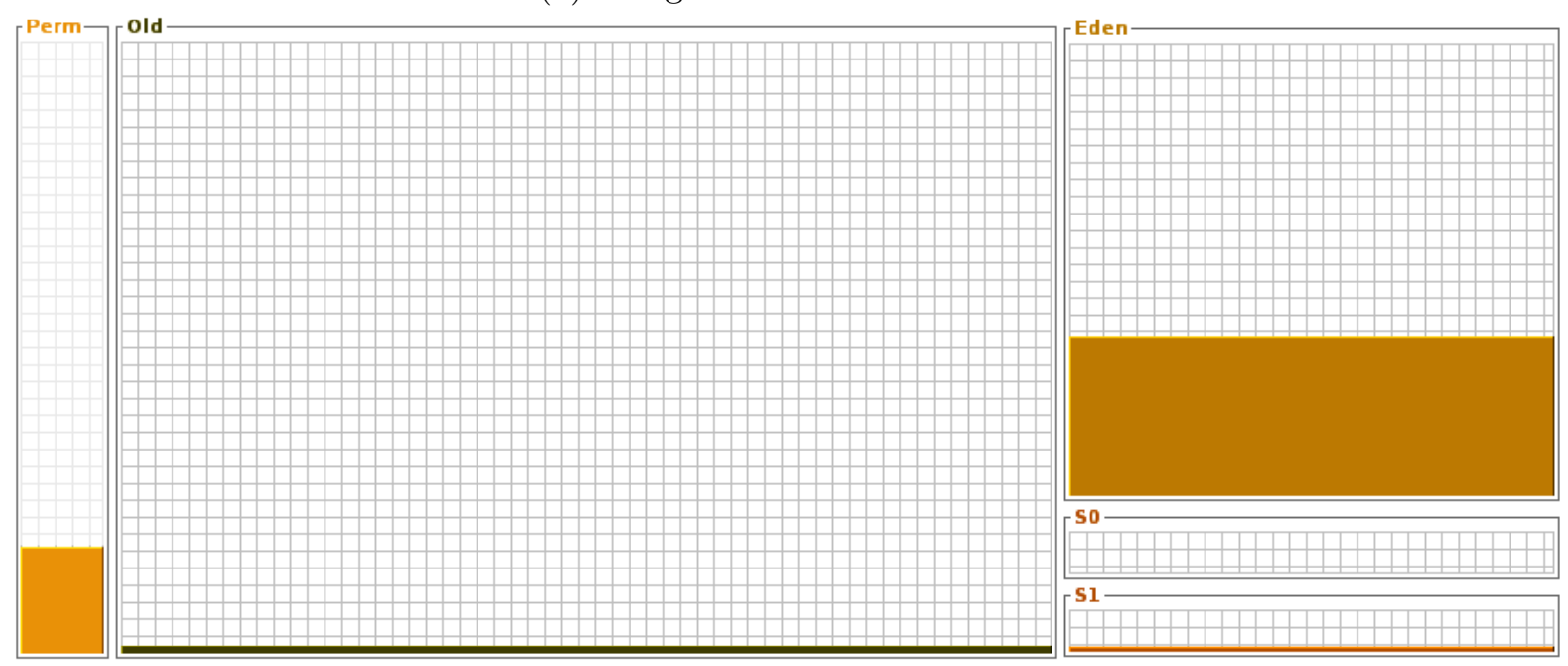

(b) GC generations after test 


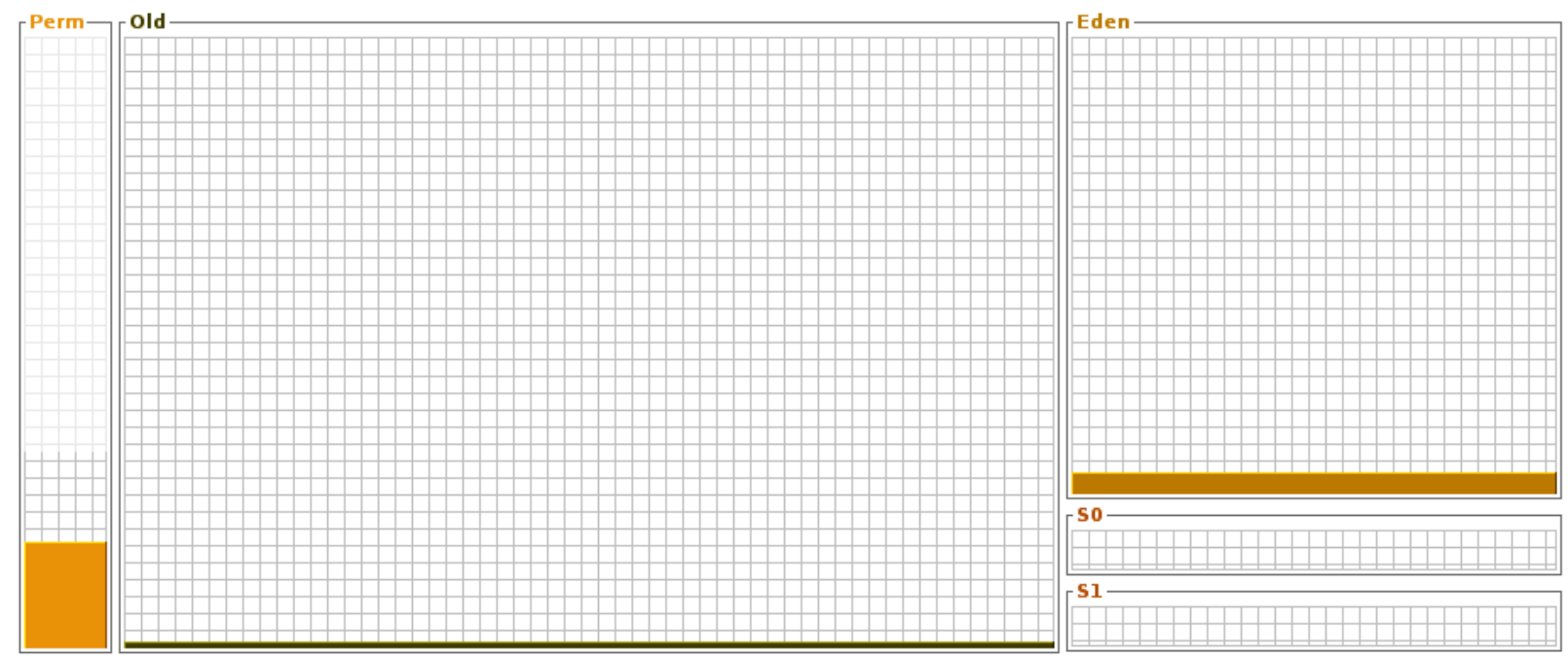

(c) GC generations after full GC

Figure 5.8: ONOS GC generations in throughput test

Figures 5.8a, 5.8b, and 5.8c present the result of the second test for ONOS obtained with the VisualVM profiler. Figure 5.8a depicts the generations before the start of the test. Old generation and survivor spaces are empty, as they should be, and Eden space usage is around 500 MB. The JVM allocated $100 \mathrm{MB}$ to the permanent generation. However, as presented in Figure 5.8b, with the start of the test, JVM increased it to 200 MB.

As shown in Figure 5.8b, after the test, the usage of old generation is about $190 \mathrm{MB}$, and S1 shows usage of 66 MB. In Figure 5.8c, after performing a full GC, the size of old generation reduced to $149 \mathrm{MB}$. Also, the full GC made S0 and S1 spaces empty.

Tracing and identifying memory leaks can be difficult, and time-consuming. However, examining memory management of OpenDaylight and ONOS with two different profilers indicate they behave normally throughout the garbage collection cycle, and there is no sign of a memory leak. 


\subsection{CPU Usage Results}

Aside from memory, YourKit also provides CPU utilization of the profiled application. While investigating the memory usage of controllers, we noticed OpenDaylight's CPU usage was extremely high compared to ONOS. This behavior of OpenDaylight motivated some additional follow-up tests on both controllers to understand and compare their CPU utilization under throughput test.

Along with YourKit, we used netdata [9], a powerful tool for real-time performance monitoring, to capture the CPU utilization of ONOS and OpenDaylight under the throughput test. We conducted our follow-up throughput tests on ONOS and OpenDaylight with 1 to 64 switches. The tests were run on the physical host with HT enabled.

We used isolcpus command to isolate the CPUs, and the taskset command to bind them to controllers at run time. We used 16 threads to run each controller. Hence, the graphs present total CPU utilization of controllers in each test with 16 threads. Each CPU usage graph shows the average from three runs.

In the CPU utilization graphs, the horizontal axis of each graph shows time in seconds. The scale of this axis is from 0 to 100, which indicates the duration of the test. The vertical axes show CPU utilization percentage for controllers under the throughput test. The vertical axis scale ranges from 0 to 100 .

Figure 5.9 shows ONOS and OpenDaylight CPU utilization under throughput test with 1 to 64 switches, respectively. These graphs appear over the next 7 pages.

Figure $5.9 \mathrm{~b}$ shows that OpenDaylight's total CPU usage is around $40 \%$ with only one connected switch. This utilization is extremely high compared to ONOS, which only consumes $7 \%$ of its total CPU. With two connected switches, ONOS CPU usage is about $14 \%$. However, as shown in Figure $5.9 \mathrm{~d}$, OpenDaylight utilizes about $50 \%$ of its allocated CPU with two switches. With four switches, ONOS CPU usage doesn't exceed 25\%. On the other hand, OpenDaylight's CPU utilization in the same test is more than $60 \%$. 


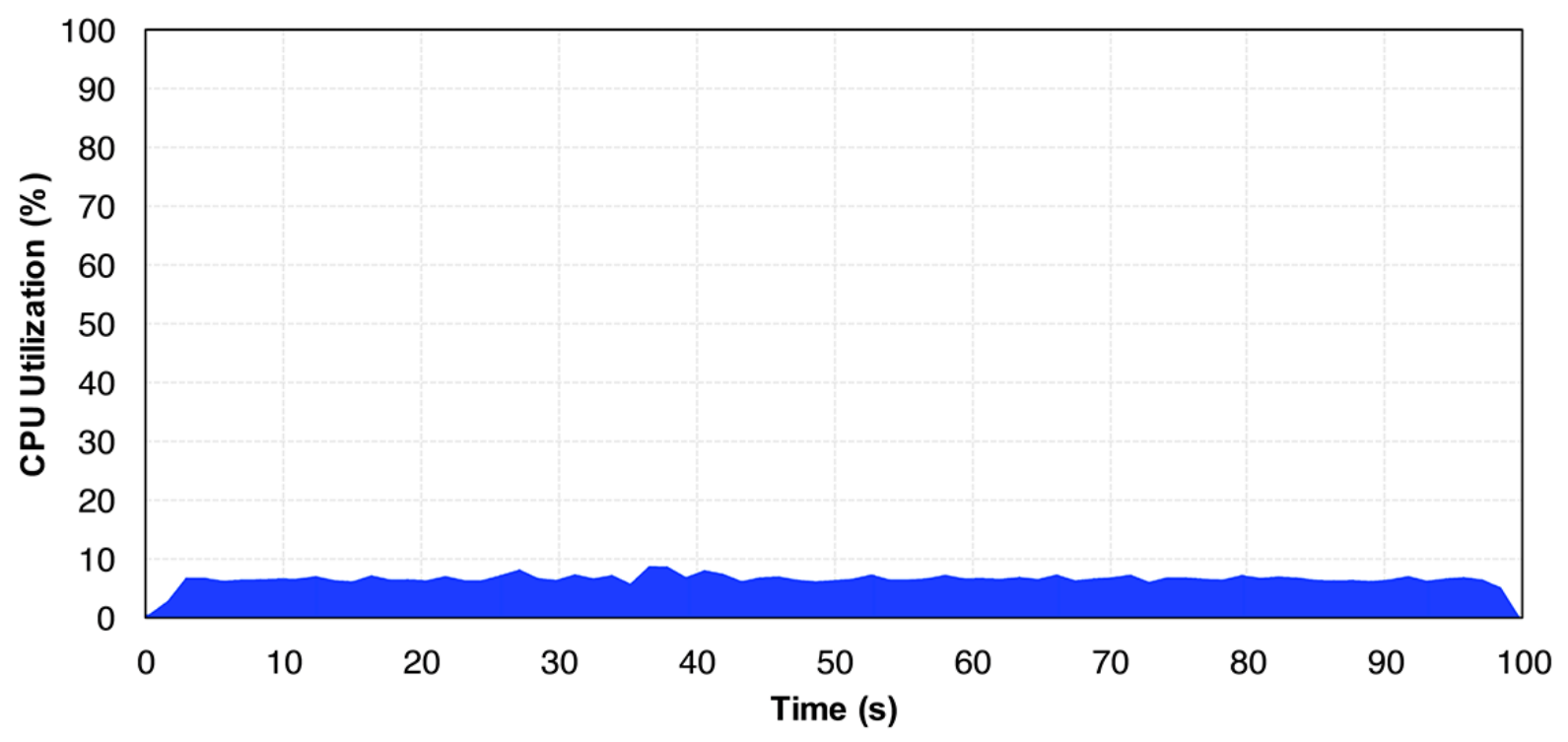

(a) ONOS CPU usage with 1 switch

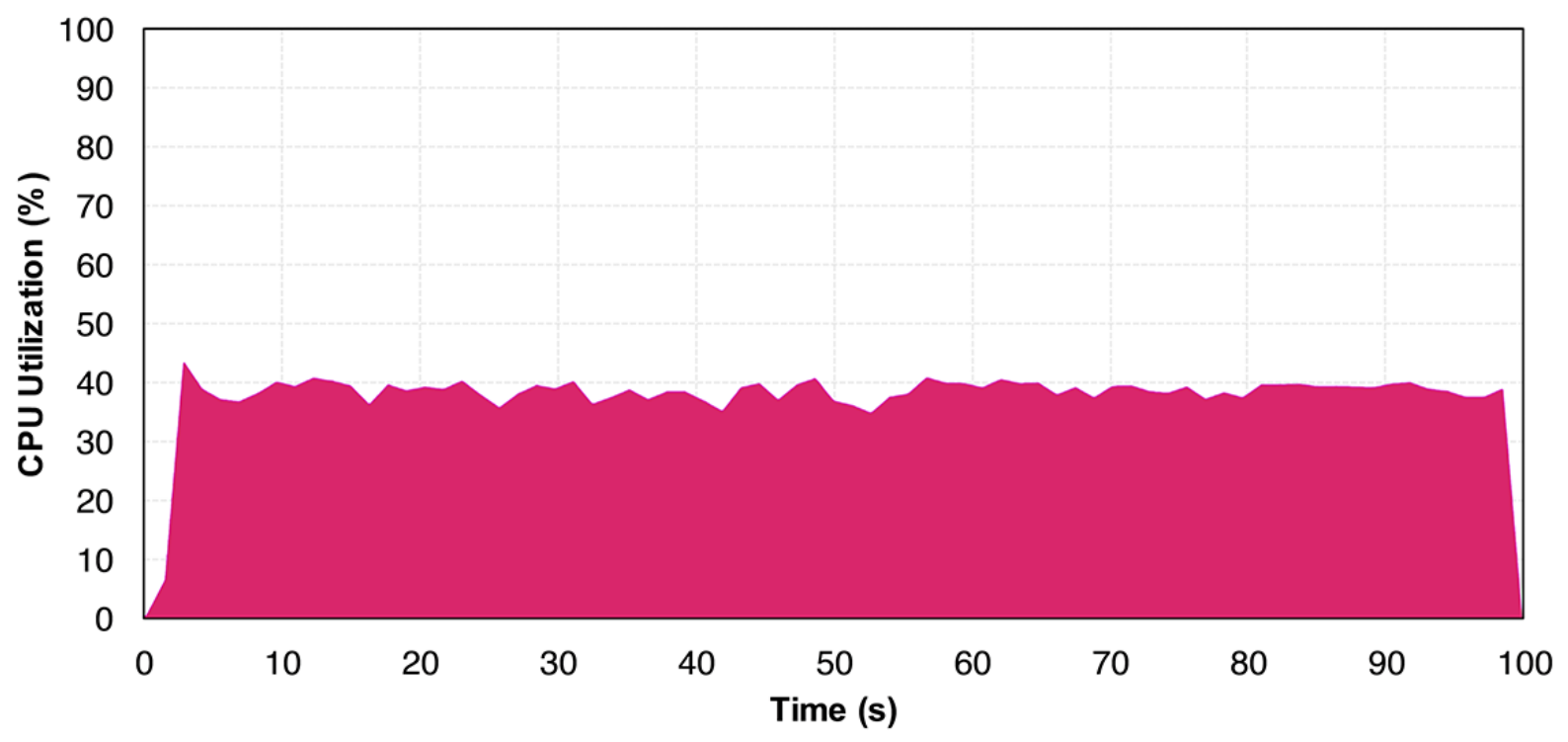

(b) OpenDaylight CPU usage with 1 switch 


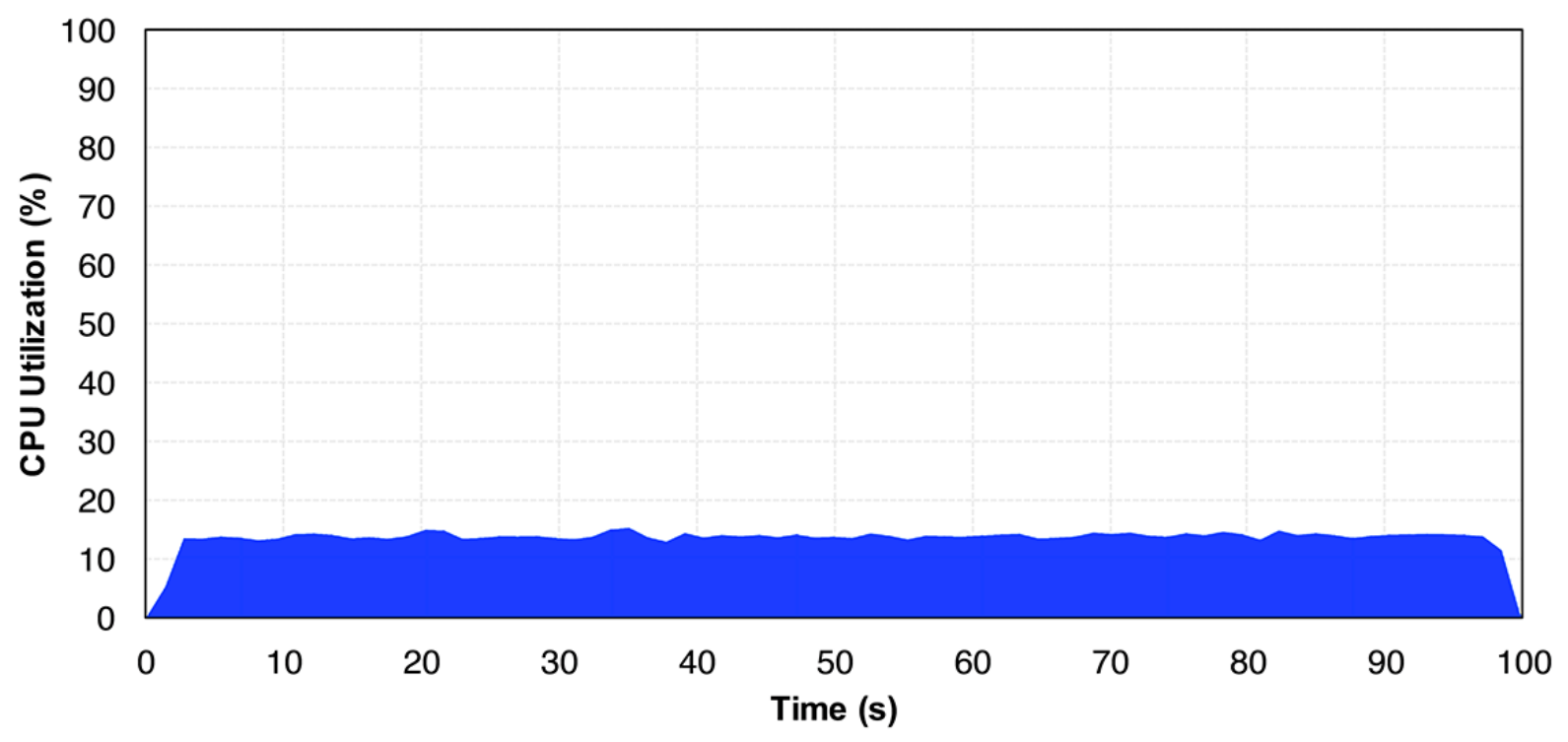

(c) ONOS CPU usage with 2 switches

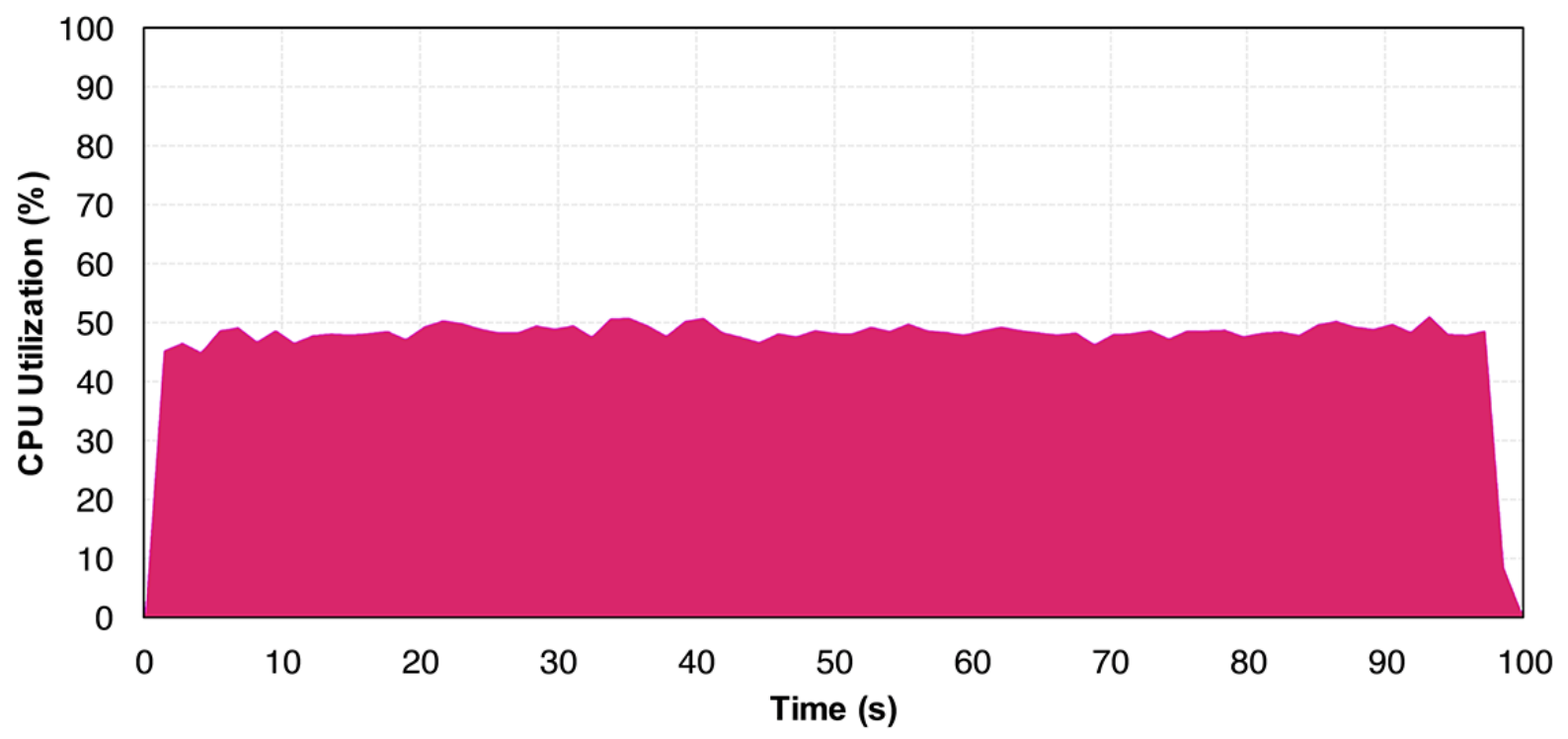

(d) OpenDaylight CPU usage with 2 switches 


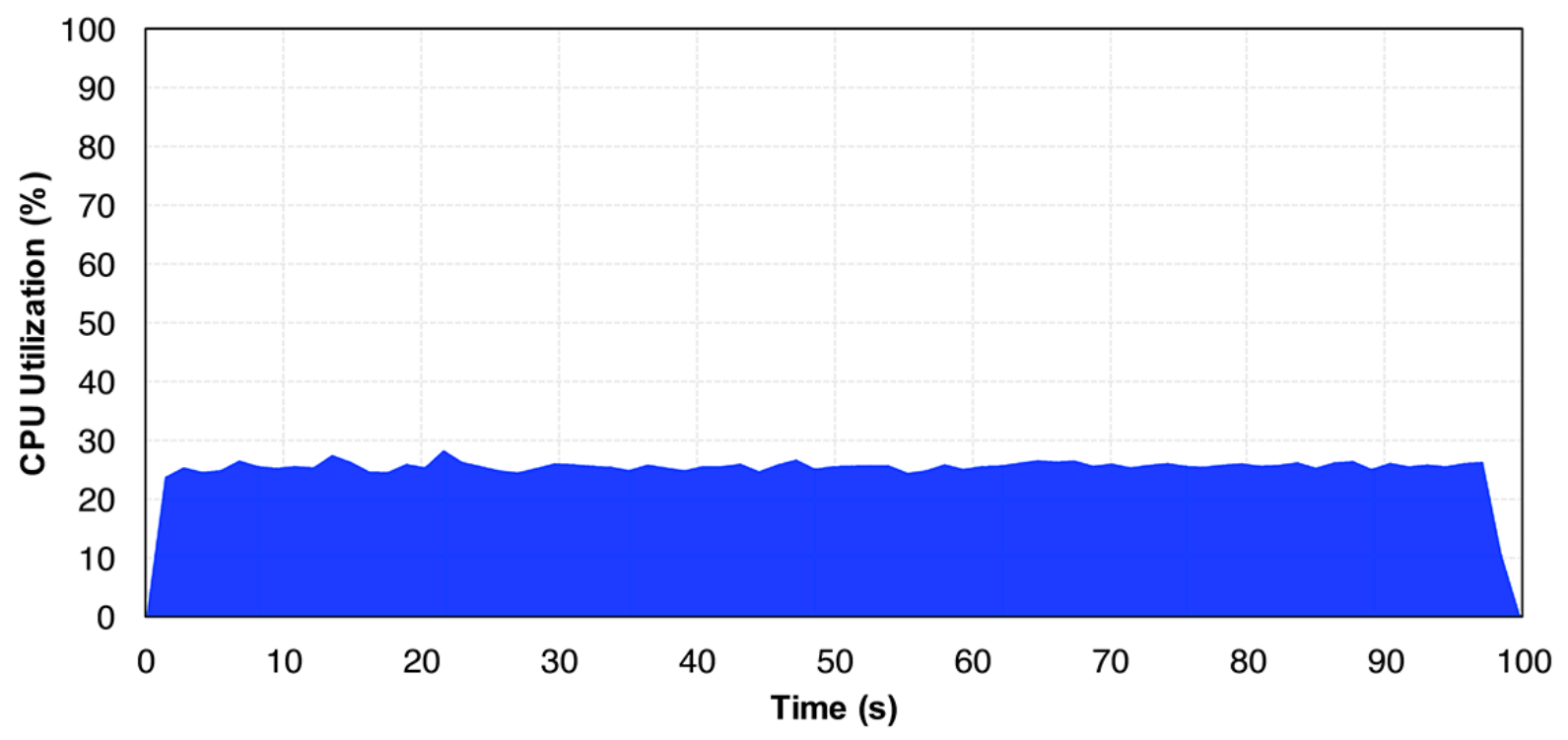

(e) ONOS CPU usage with 4 switches

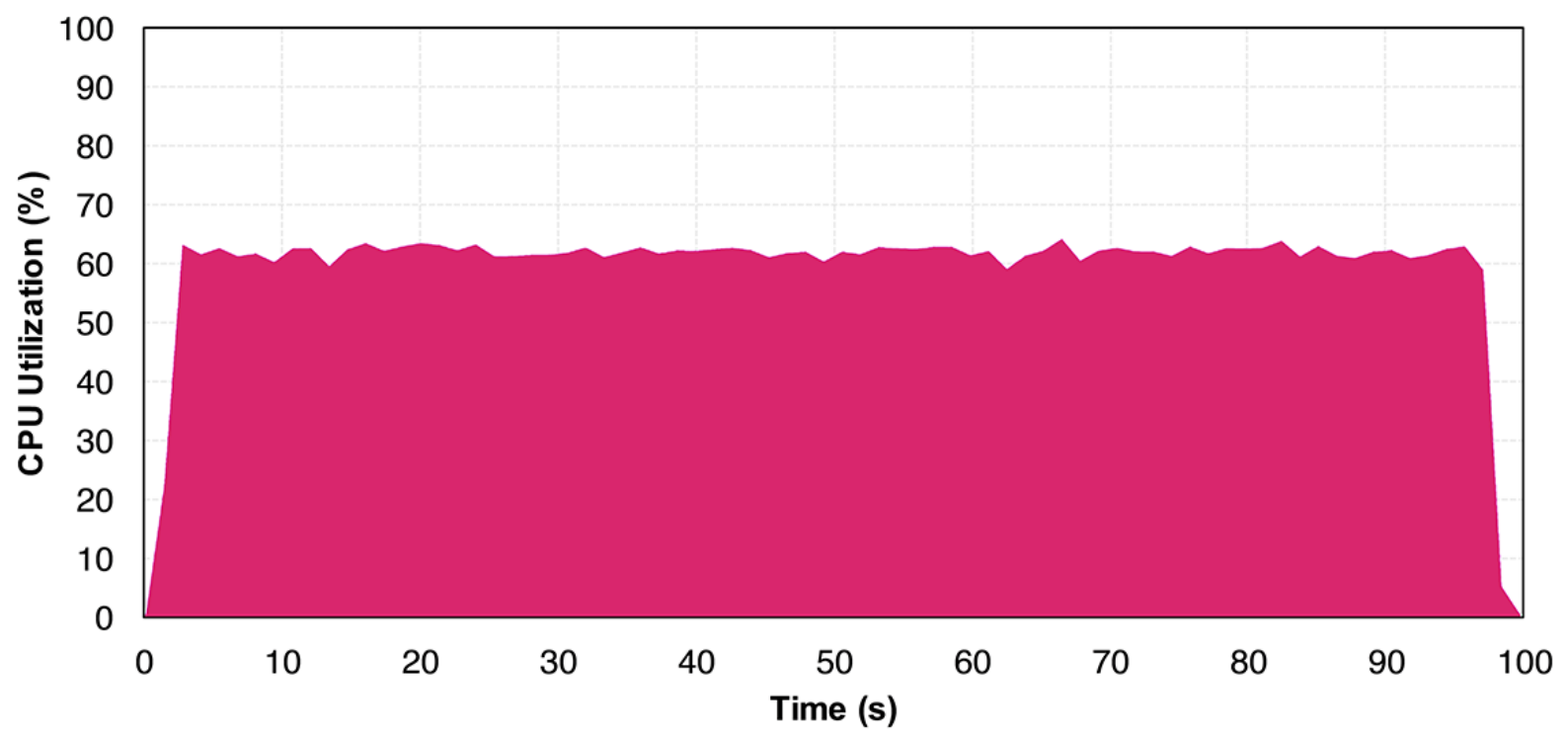

(f) OpenDaylight CPU usage with 4 switches 


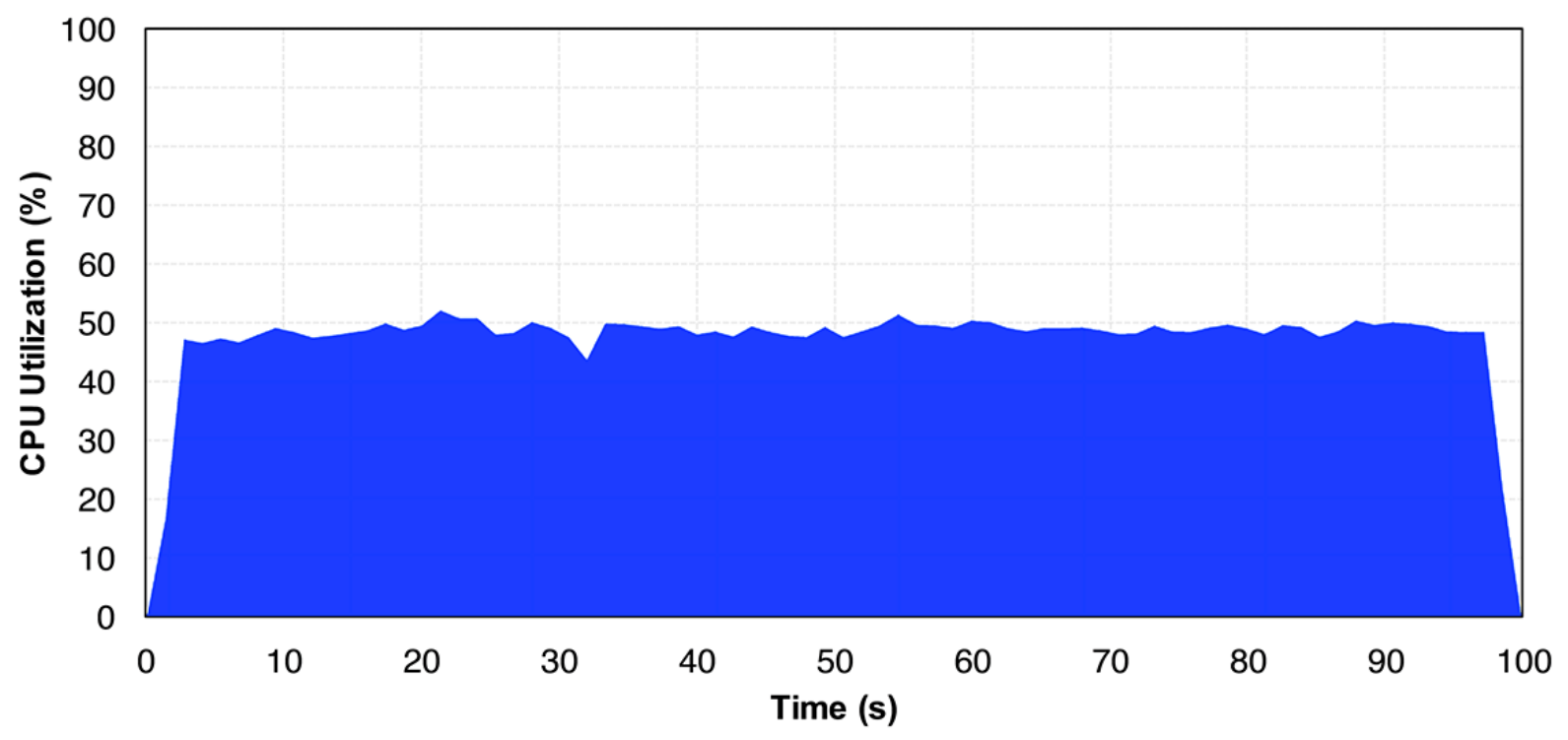

(g) ONOS CPU usage with 8 switches

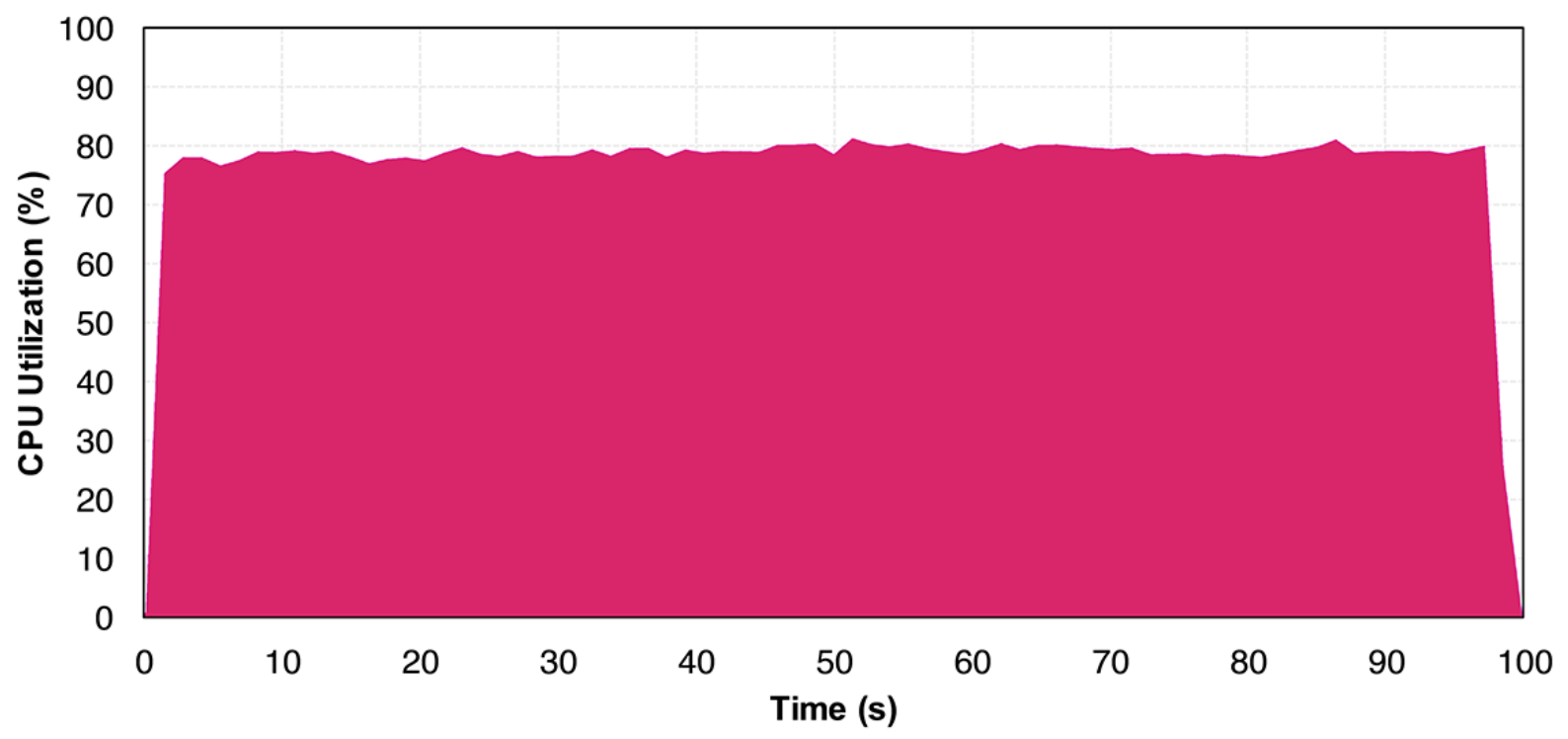

(h) OpenDaylight CPU usage with 8 switches 


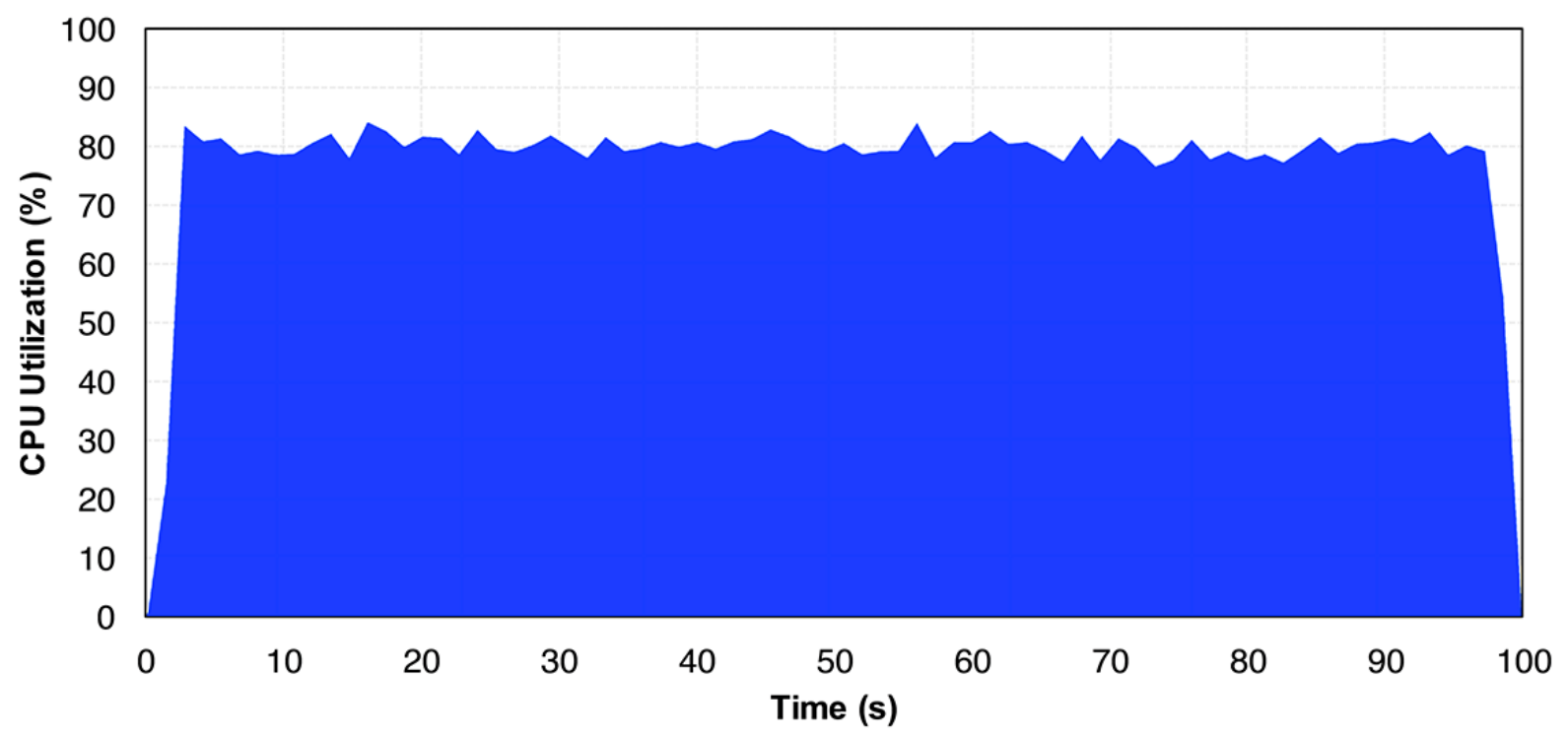

(i) ONOS CPU usage with 16 switches

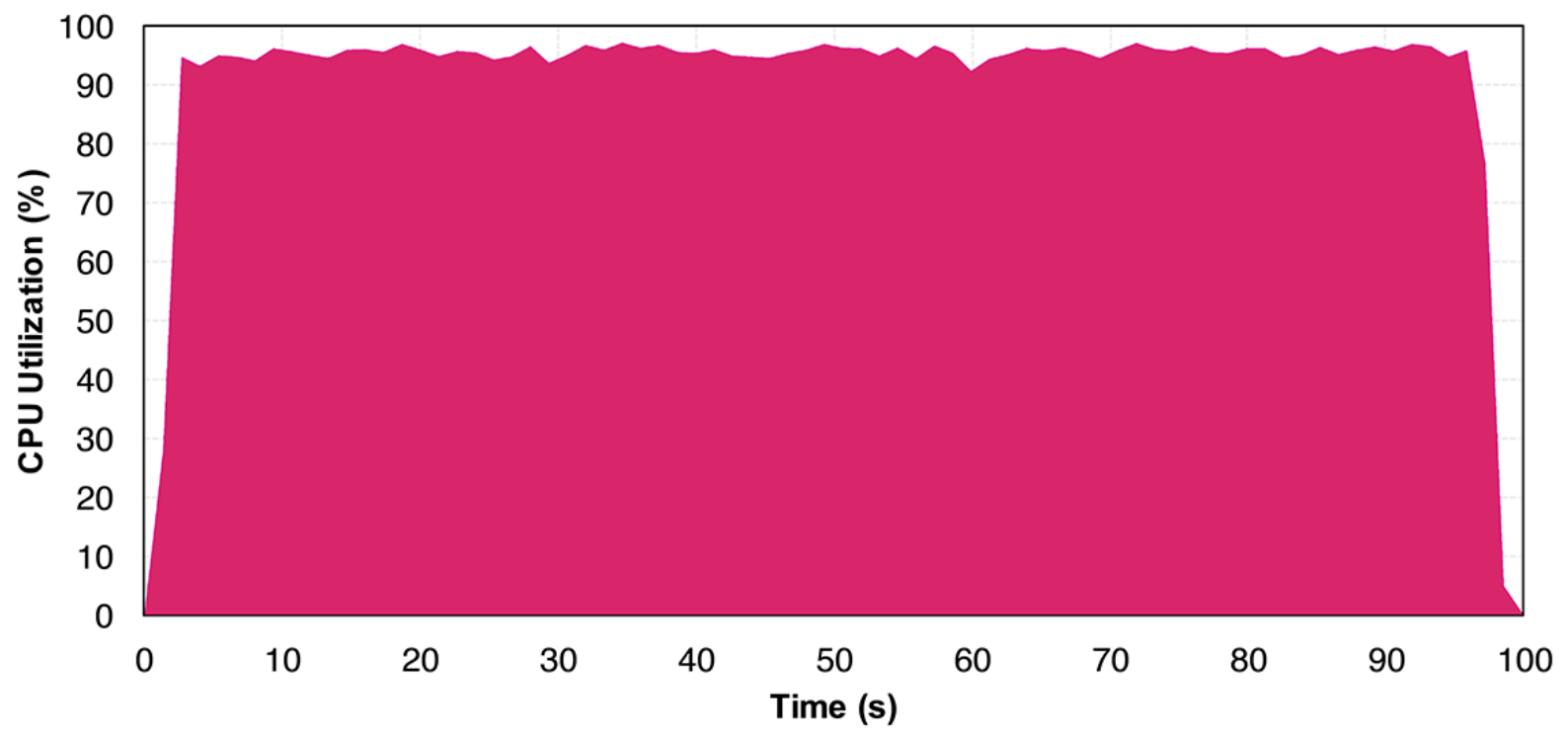

(j) OpenDaylight CPU usage with 16 switches 


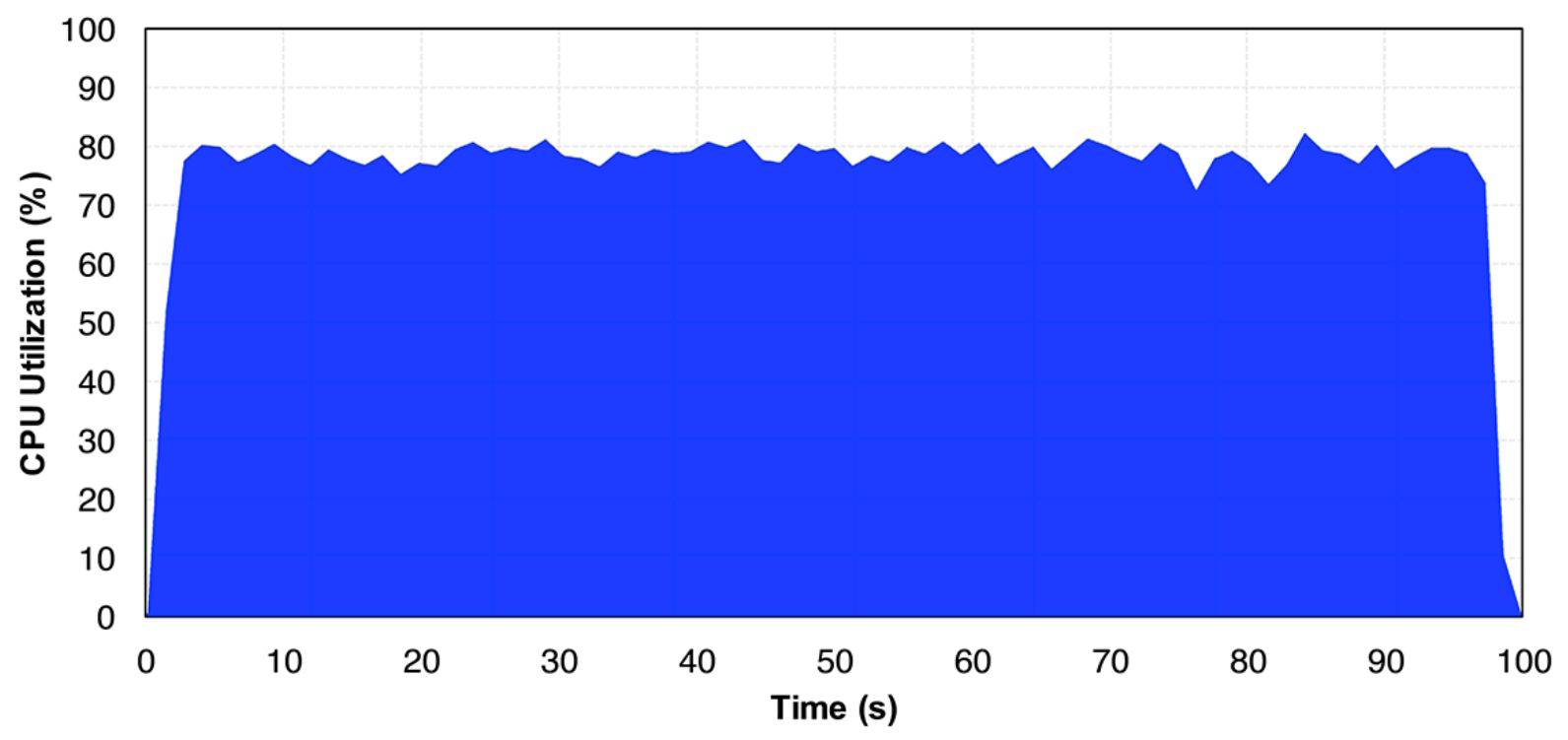

(k) ONOS CPU usage with 32 switches

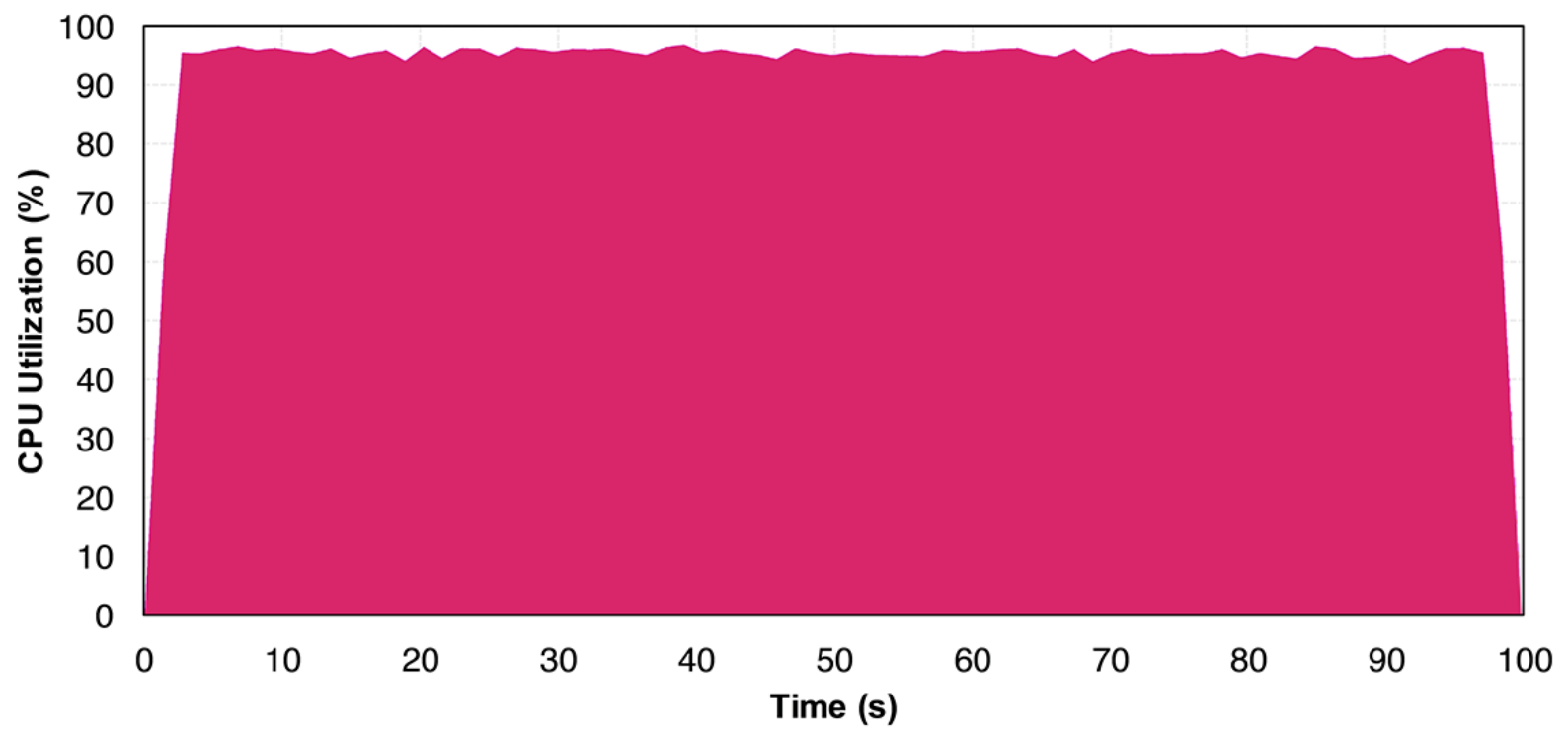

(l) OpenDaylight CPU usage with 32 switches 


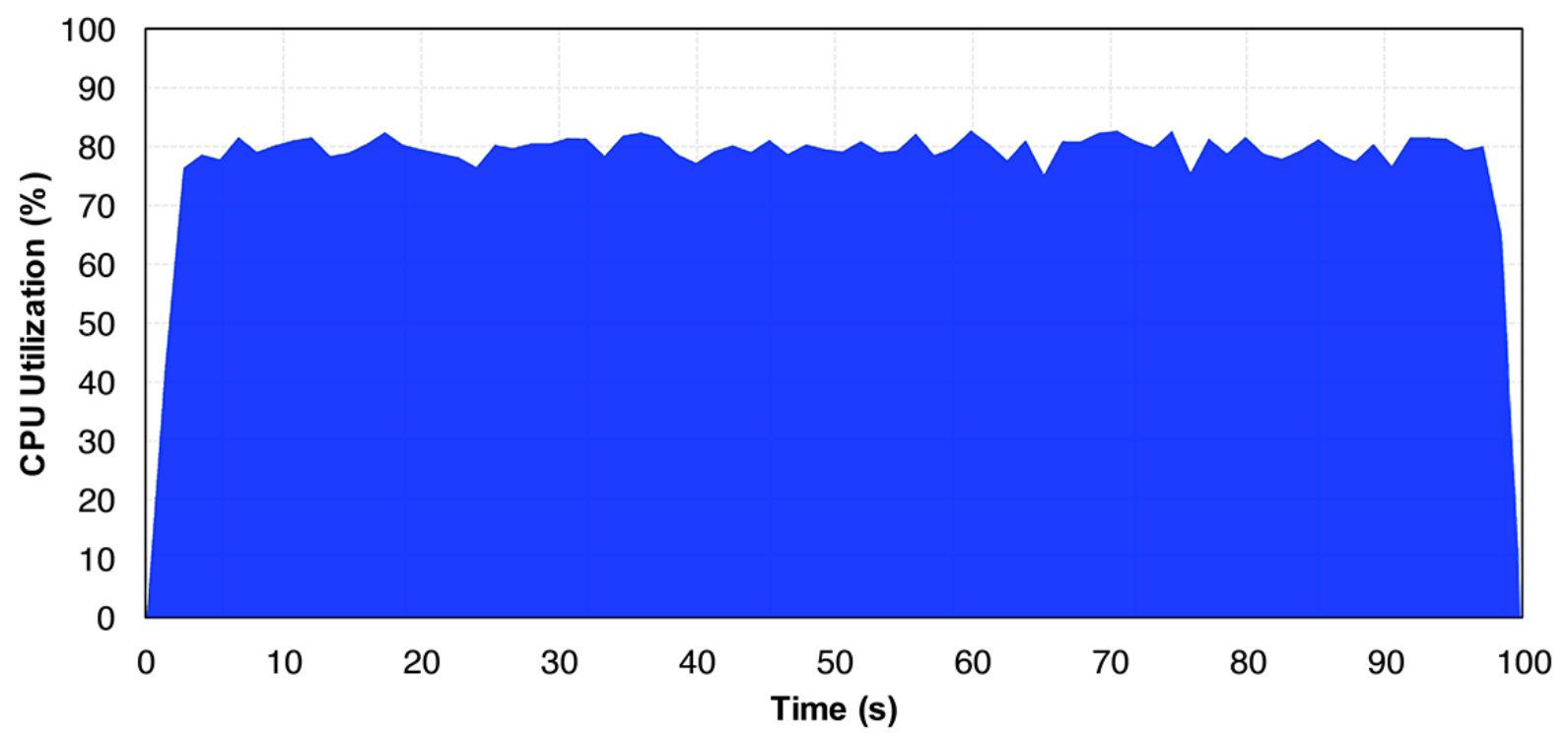

(m) ONOS CPU usage with 64 switches

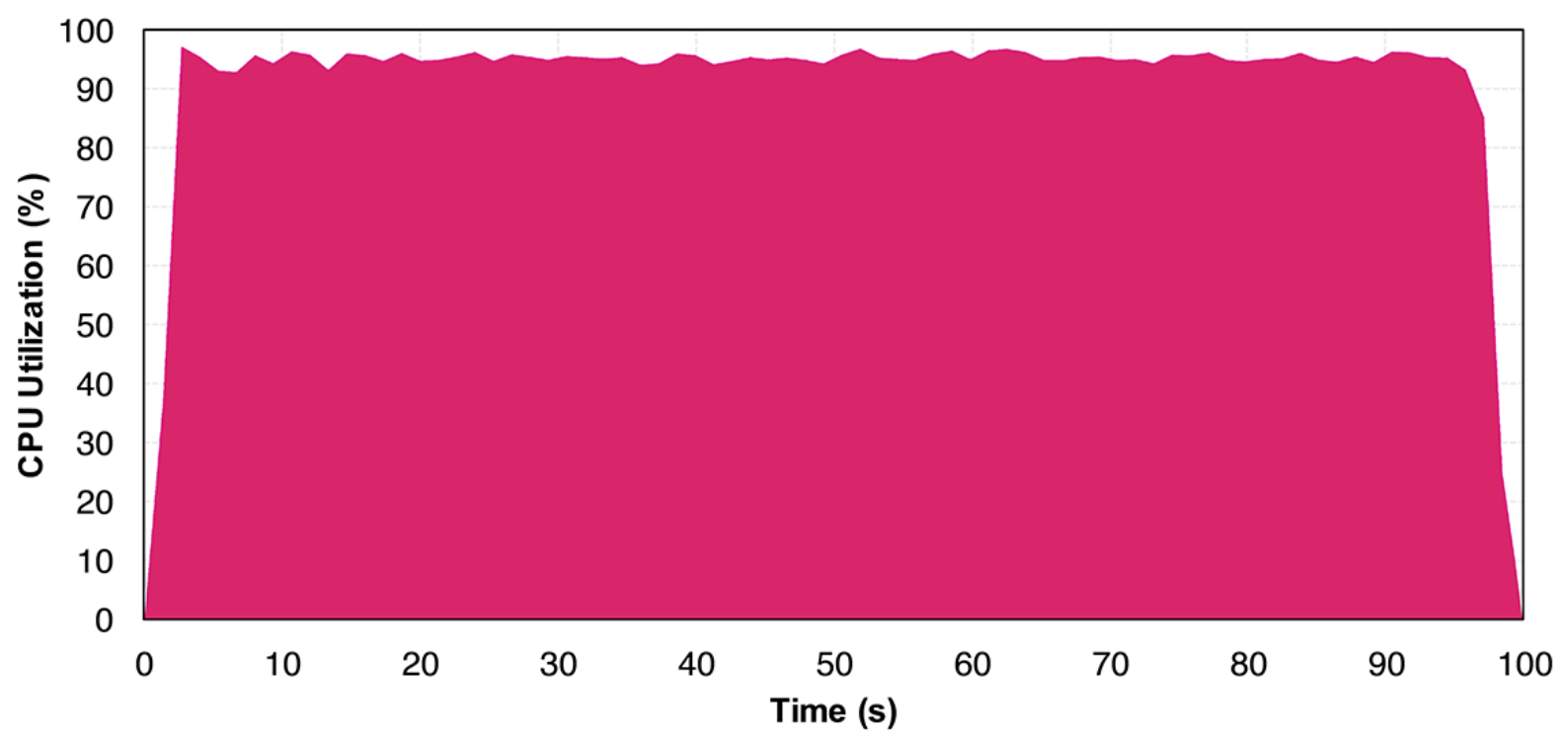

(n) OpenDaylight CPU usage with 64 switches

Figure 5.9: ONOS and OpenDaylight CPU usage under throughput test 
As shown in Figure 5.9g, ONOS's CPU usage reaches 50\% with eight connected switches. OpenDaylight's CPU usage, with the same number of switches, is about $80 \%$. ONOS's CPU utilization stays around 80\% for 16 switches (and more). Conversely, OpenDaylight's CPU usage exceeds $96 \%$ for 16 switches and above. As shown in Figure 5.9j, OpenDaylight saturates the CPU. Hence, the decline in throughput is expected.

The total CPU usage of OpenDaylight clearly indicates that the main reason behind its poor performance in the throughput test is a CPU-related problem rather than memory leakage. Considering that ONOS and OpenDaylight are both Java-based controllers (same programming language), and use identical NIO libraries (Netty), they should have similar performance.

OpenDaylight's surprisingly high CPU utilization, even with one connected switch, suggests a possible bug in OpenDaylight's OpenFlow plugin. Moreover, since the issue is exaggerated by an increase in the number of switches, it is likely that either the Switch Partitioning algorithm or the Packet Batching (packet-in scheduling) method are the problem. They do not seem well optimized, which severely affects the controller's CPU utilization and reduces the throughput.

The Switch Partitioning algorithm allocates and distributes connected OpenFlow switches to worker threads running in the controller. Packet Batching is a method in OpenFlow controllers to bundle multiple bytes while reading from or writing to an underlying network. Its purpose is to prevent frequent system calls, and improve the overall throughput.

Considering the throughput and latency test results, OpenDaylight performs better in latency tests. Even though its performance is still lower than ONOS, OpenDaylight's OpenFlow plugin functions much better with a low volume of packet_in messages. Whereas, when it receives a burst of packet_in messages in the throughput test, it fails to respond to many of them. Hence, aside from the OpenFlow plugin, this behavior suggests the Switch Partitioning algorithm employed in the OpenDaylight controller needs to be optimized. 
It is worth noting that Maestro, which is also a Java-based and multi-threaded controller, showed throughput degradation in a previous study [83]. Maestro provided a throughput of $4 \mathrm{M}(\mathrm{resp} / \mathrm{s})$ with 32 switches. However, it demonstrated a drastic throughput decrease when increasing the number of switches beyond 32. Its throughput dropped to less than $100 \mathrm{~K}(\mathrm{resp} / \mathrm{s})$ with 256 switches. The authors stated that this behavior of Maestro is mainly due to its packet distribution algorithm.

\subsection{Thread Scalability Results with Threads on One Socket}

In Section 4.4 of Chapter 4, we observed a slight decline in throughput with 12-15 threads. We explained the trend and its cause. However, we conducted two follow-up experiments with threads on one processor socket to discover the thread scalability of the controllers without the overhead of inter-socket communication.

As mentioned in Chapter 3, the server used to run the controllers has two processor sockets. When HT is enabled, the server has a total of 48 threads, including 24 threads per socket. Threads 0-11 and 24-35 reside on socket one. On the other hand, threads 12-23 and 36-47 reside on socket two. We run both experiments on the physical host with HT enabled, and used 16 threads from socket one. We isolated threads 0-11 and 24-27, and pinned them to controllers at run time.

Figures 5.10 and 5.11 present the average throughput of ONOS and OpenDaylight with different numbers of threads. The yellow line in each graph shows the throughput results with threads on two processor sockets. On the other hand, the purple line illustrates the average throughput with threads on one socket.

Both controllers show a higher throughput when all threads are on one socket, compared to their performance with threads on two sockets. Also, with threads on one socket, we do not observe the throughput decline in threads 13-15. 


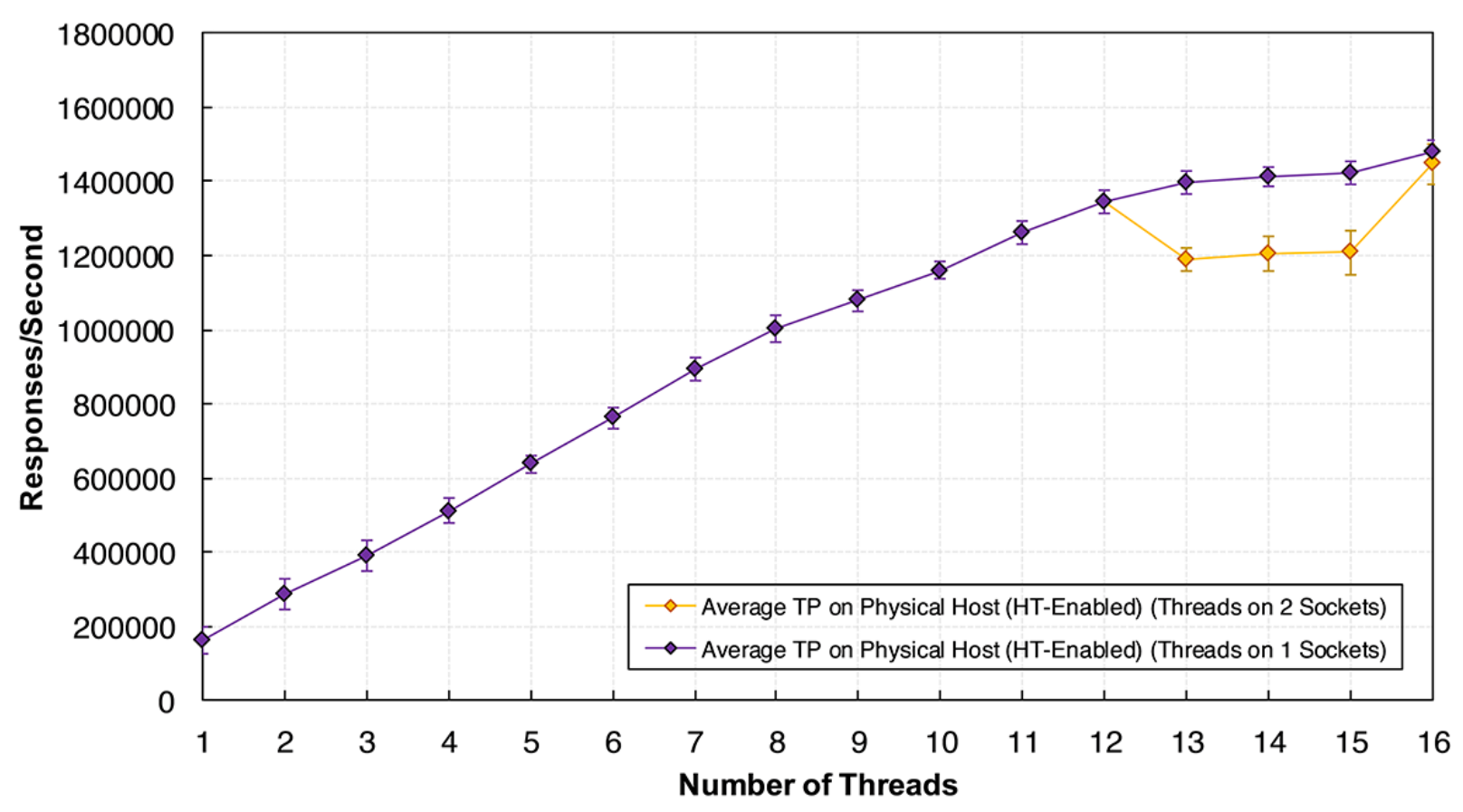

Figure 5.10: ONOS average TP with different number of threads (1 socket vs 2 sockets)

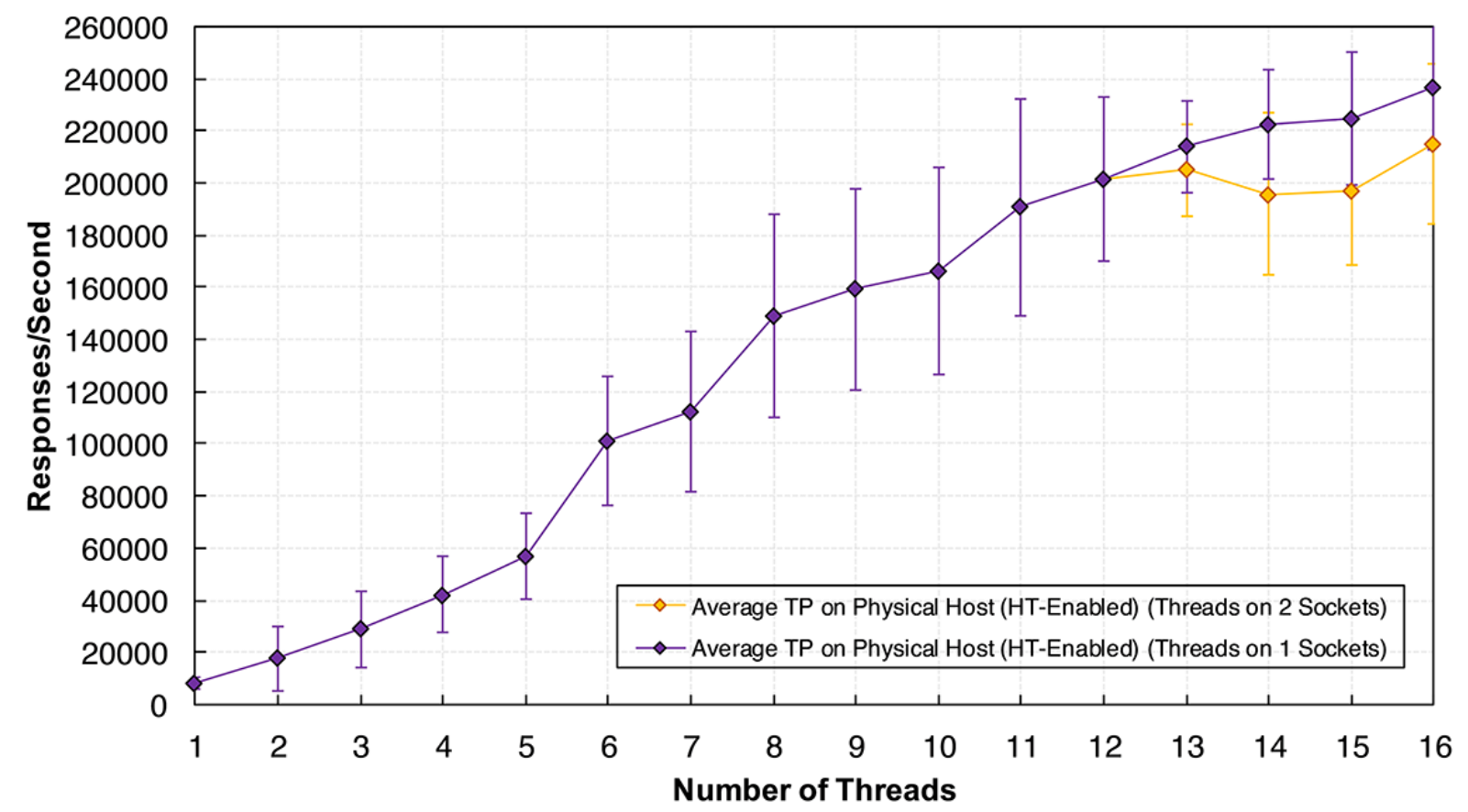

Figure 5.11: ODL average TP with different number of threads (1 socket vs 2 sockets) 
As shown in Figure 5.10, with 13 threads, ONOS's average throughput is $1.4 \mathrm{M}$ (resp/s). The throughput with $14-15$ threads is slightly better. The average throughput is $1.41 \mathrm{M}$ $(\mathrm{resp} / \mathrm{s})$, and 1.42M (resp/s), with 14, and 15 threads, respectively.

For ONOS, with threads on two sockets, the throughput with $13-15$ threads is $200 \mathrm{~K}$ $(\mathrm{resp} / \mathrm{s})$ lower. ONOS's average throughput with 16 threads, on one socket, is $1.48 \mathrm{M}$ (resp/s). ONOS showed the throughput of 1.44M (resp/s) when threads were on two sockets. ONOS's error bars with 13-16 threads are smaller when the threads are on one socket.

OpenDaylight showed a similar trend to ONOS. As shown in Figure 5.11, OpenDaylight's throughput with 13 threads is $214 \mathrm{~K}(\mathrm{resp} / \mathrm{s})$, which is higher than $205 \mathrm{~K}(\mathrm{resp} / \mathrm{s})$ obtained with threads on two sockets.

The throughput with $14-15$ threads is $222 \mathrm{~K}$ (resp/s), and $224 \mathrm{~K}$ (resp/s), respectively. OpenDaylight's throughput with the same number of threads was 195K (resp/s), and 196 (resp/s), respectively. The performance of both controllers in this test indicates that their throughput reaches a plateau with 13-15 threads, and does not change significantly.

With 16 threads, OpenDaylight shows a throughput of 236K (resp/s) with all threads on one socket, which is $22 \mathrm{~K}$ ( $\mathrm{resp} / \mathrm{s}$ ) higher than its throughput with 16 threads on two sockets. We observed OpenDaylight's error bars with 13-16 threads are smaller when all threads reside on one socket.

\subsection{Summary}

In this chapter, we studied ONOS and OpenDaylight memory and CPU utilization. In Section 5.1, with the use of two different tools, namely YourKit and VisualVM, we scrutinized memory management of both controllers. We did not observe any sign of memory leak,

and both controllers showed normal memory management behavior. In Section 5.2, we investigated CPU usage, which revealed the cause of OpenDaylight's poor performance in the throughput test. Lastly, in Section 5.3 , we presented the results of our follow-up experiments 
on the thread scalability of controllers when all threads reside on one processor socket. The next chapter summarizes the outcome of this thesis, and suggests future work directions. 


\section{Chapter 6}

\section{Conclusions}

This thesis presents experimental evaluation of the throughput, latency, and scalability aspects of two state-of-the-art open-source OpenFlow controllers, namely ONOS and OpenDaylight. Section 6.1 provides a summary of this thesis. Conclusions are discussed in Section 6.2. Lastly, future work is presented in Section 6.3.

\subsection{Thesis Summary}

In Chapter 1, we described the importance of software-defined networking. Specifically, we discussed OpenFlow and the importance of a responsive OpenFlow controller in today's complex networks. Then we highlighted ONOS and OpenDaylight among existing SDN controllers, due to their extensive features. Our work was motivated by the lack of an unbiased recent benchmarking study on the performance of these controllers. Then we stated our objectives and highlighted our primary contributions.

In Chapter 2, we reviewed the background information related to this thesis. We described the architecture, benefits, and challenges of SDN, as well as an introduction to OpenFlow and its communication messages. Then we discussed ONOS and OpenDaylight

and their use cases. We also outlined Intel Hyper-threading technology and the prominence of virtualization, with a particular concentration on OpenStack as our virtualization platform in this work. Lastly, we summarized recent studies involving benchmarking and performance evaluation of OpenFlow controllers.

Chapter 3 presented our benchmarking methodology including testbed setup and software tools. We identified throughput, latency, and thread scalability as our primary metrics in this experimental study. Also, we discussed our experimental methodology and standard 
methods for analysis of results.

Chapter 4 presented the results. We presented and compared the throughput and latency of ONOS and OpenDaylight under various test situations. When possible, we provided comparison to prior work to obtain a clearer understanding of their performance.

In Chapter 5, we also investigated memory usage of both controllers with the use of code profiling, which showed both controllers have reasonable memory management structure. Furthermore, by observing CPU utilization of OpenDaylight under the throughput test, we identified a performance bottleneck. Lastly, we showed improved thread scalability of ONOS and OpenDaylight when threads reside on one socket instead of two sockets.

\subsection{Conclusions}

In this thesis, we learned that the performance of an OpenFlow controller depends on many different factors. The key factors are underlying infrastructure, the number of threads, distribution of threads between sockets, controller's configuration, etc. Also, we learned that the performance might vary a lot across different versions of a controller.

Our experimental evaluation of ONOS and OpenDaylight shows that enabling Hyperthreading results in performance improvement for both controllers. Overall, in most of the tests, ONOS and OpenDaylight show higher throughput and lower latency when Hyperthreading is enabled.

Our study showed that the performance of ONOS and OpenDaylight differ on the virtual host. ONOS's throughput on the virtual host is about $13.5 \%$ lower than its throughput on the physical host. On the other hand, OpenDaylight's throughput on the virtual host is similar to its throughput on the physical host. Both controllers had flow setup latency on the virtual host that was two times higher than on the physical host.

Our experimental evaluation of ONOS and OpenDaylight indicates that ONOS outperforms OpenDaylight for throughput and latency. Overall, ONOS shows more scalable and 
robust performance than OpenDaylight. OpenDaylight's behavior in throughput tests suggests a possible bug in its OpenFlow plugin.

Finally, our experimental evaluation of ONOS and OpenDaylight shows that they do not deliver the highest throughput and lowest latency among all multi-threaded controllers. This result may be due to I/O libraries, Packet Batching techniques, and packet distribution algorithms employed in these controllers. However, our evaluation of thread scalability indicates that they can effectively use more threads than other controllers.

\subsection{Future Work}

In this work, we focused on evaluating the performance of ONOS and OpenDaylight. However, we have also unveiled several avenues for future research. Below are a few recommendations for future directions:

- Cluster Mode: In this work, we focused on the evaluation of the controllers in the single-instance mode to create a baseline for the performance of single-node ONOS and OpenDaylight. Even though we can extrapolate the performance of a cluster from a single-instance controller, there are other dimensions to be considered for evaluation. Some are only available in cluster mode, such as controller availability, or scalability and performance when the nodes interact with each other in complex ways.

- Southbound APIs: Although OpenFlow is the most frequently implemented Southbound API, in some cases deployment of OpenFlow is not an option. For instance, in some legacy networks or service provider environments, the existing equipment is not OpenFlow-enabled. Hence, shifting to software-defined networking would only be possible with the use of another Southbound protocol supported by their infrastruc-

ture, such as NetConf or SNMP. It would be useful to have a comprehensive study of ONOS and OpenDaylight performance with other Southbound protocols. 
- ProActive Flow Setup: In this work, we used Cbench to assess the controllers based on reactive flow entry. We did not pre-populate the controllers with flow entries or rules. However, it would be worthwhile to evaluate ONOS and OpenDaylight response time when we have configured the desired path in advance. This experiment would require having two end hosts, some OpenFlow switches, and multiple paths between hosts with different priorities. While hosts are communicating, the main link that connects end hosts should be disconnected. Since there are multiple paths between end hosts, we can gauge responsiveness of the controllers to automatically re-route the traffic in case of path removal or network failure.

- Plugin-enabled and Scenario-based Performance: Unlike centralized SDN controllers, ONOS and OpenDaylight have numerous features and plugins to integrate with Northbound applications and orchestrators, or with Southbound forwarding devices, network protocols, and services. Enabling each of these features might negatively impact the performance of the controllers. To date, benchmarking OpenFlow controllers has been conducted on controllers with their most optimized configuration, without any overhead from apps and plugins that might be essential for the underlying network requirements. One possible direction to expand this work could be evaluating ONOS and OpenDaylight under different scenarios that would be used by service providers (MPLS, BGP, service load balancing) or cloud and NFV providers (automation, resource optimization).

- Next-generation Cbench: Even though Cbench is commonly used in numerous benchmarking studies, we believe it is time to investigate and implement a new benchmark toolset to examine various performance aspects of distributed controllers without Cbench's limitations. Below we list some of the limitations of Cbench that we observed in this study: 
- First of all, Cbench only uses one CPU thread, regardless of the number of threads available. In our case, use of only one thread saturated the I/O capacity of the controller. Using a single instance of Cbench would not be very efficient for benchmarking distributed controllers. Furthermore, running multiple instances of Cbench in parallel from different servers to stress the controller and combine the achieved responses from all instances may not result in an accurate evaluation. The next generation of Cbench should be a distributed benchmarking platform comprised of one master node and a configurable number of agents. The agents run on different servers and collect the responses, then integrate with the master node to calculate the total throughput.

- Secondly, Cbench does not allow us to emulate a larger number of OpenFlow switches (e.g., 1000 switches or above). It was not our concern in this study, since we evaluated a single controller. However, to evaluate ONOS, OpenDaylight, or any distributed controller in cluster mode, we would need to simulate distributed data centers or enterprise networks with more switches.

- Lastly, Cbench only supports OpenFlow as the Southbound API for benchmarking throughput and latency of an SDN controller. It would be nice to include other Southbound APIs (e.g., NetConf, SNMP) in the next generation of Cbench. 


\section{Bibliography}

[1] Cbench (OpenFlow Controller Benchmarking Tool). https://github.com/ andi-bigswitch/oflops/tree/master/cbench.

[2] Composite Standard Deviation. http://www.burtonsys.com/climate/composite_ standard_deviations.html/.

[3] Floodlight. http://floodlight.openflowhub.org/.

[4] How Do the CPUs on Different Sockets Communicate? http://stackoverflow.com/ questions/12202488/how-do-the-cpus-on-different-sockets-communicate.

[5] HT and Turbo Boost Benefits. https://www.techpowerup.com/reviews/Intel/ Core_i5_661/14.html.

[6] Hunting Memory Leaks in Java. https://www.toptal.com/java/ hunting-memory-leaks-in-java.

[7] iPerf - The ultimate speed test tool for TCP, UDP and SCTP. https://iperf.fr/.

[8] Microsoft Hyper-V. https://en.wikipedia.org/wiki/Hyper-V.

[9] Netdata - Distributed Real-Time Performance and Health Monitoring. https:// github.com/firehol/netdata.

[10] ONF to standardize northbound API for SDN applications? http://searchsdn.techtarget.com/news/2240206604/ ONF-to-standardize-northbound-API-for-SDN-applications.

[11] Open Network Operating System. http://onosproject.org/.

[12] Open Networking Foundation. http://www.opennetworking.org/. 
[13] Open vSwitch. http://openvswitch.org/.

[14] OpenDayLight. http://www.opendaylight.org/.

[15] OpenFlow. http://www.openflow.org/.

[16] OpenIRIS. http://openiris.etri.re.kr/.

[17] OpenMUL. http://www.openmul.org/.

[18] OpenStack. https://www.openstack.org/.

[19] Performance Tuning Guide: Java Heap Tuning Parameters. https://docs.oracle. com/cd/E19900-01/819-4742/abeik/index.html.

[20] POX. http://www.noxrepo.org/pox/about-pox/.

[21] Protocol Oblivious Forwarding (POF). http://www. poforwarding.org/.

[22] Ryu. http://osrg.github.com/ryu/.

[23] The Netty Project. http://netty.io/.

[24] Trema. http://trema.github.com/trema/.

[25] VisualVM Profiler. https://visualvm.github.io/.

[26] VMware Virtualization. http://www.vmware.com/.

[27] WCbench (Wrapped CBench). https://github.com/dfarrell07/wcbench.

[28] YourKit Java Profiler. https://www.yourkit.com/.

[29] OpenFlow Switch Specification Version 1.0.0. Open Networking Foundation (ONF), December 2009. 
[30] OpenFlow Switch Specification Version 1.5.1. Open Networking Foundation (ONF), December 2014.

[31] S. Azodolmolky. Software Defined Networking with OpenFlow. Packt Publishing Ltd, October 2013.

[32] T. Bakhshi. State of the Art and Recent Research Advances in Software Defined Networking. Wireless Communications and Mobile Computing, January 2017.

[33] B. Belter, A. Binczewski, K. Dombek, A. Juszczyk, L. Ogrodowczyk, D. Parniewicz, M. Stroiñski, and I. Olszewski. Programmable Abstraction of Datapath. In Proceedings of Third IEEE European Workshop on Software Defined Networks (EWSDN), pages 7-12, London, UK, September 2014.

[34] B. Belter, D. Parniewicz, L. Ogrodowczyk, A. Binczewski, M. Stroiñski, V. Fuentes, J. Matias, M. Huarte, and E. Jacob. Hardware Abstraction Layer as an SDN-enabler for Non-OpenFlow Network Equipment. In Proceedings of Third IEEE European Workshop on Software Defined Networks (EWSDN), pages 117-118, London, UK, September 2014.

[35] P. Berde, M. Gerola, J. Hart, Y. Higuchi, M. Kobayashi, T. Koide, B. Lantz, B. O'Connor, P. Radoslavov, W. Snow, and G. Parulkar. ONOS: Towards an Open, Distributed SDN OS. In Proceedings of Third ACM SIGCOMM Workshop on Hot Topics in Software Defined Networking (HotSDN 2014), pages 1-6, Chicago, Illinois, August 2014.

[36] G. Bianchi, M. Bonola, A. Capone, and C. Cascone. OpenState: Programming Platform-independent Stateful OpenFlow Applications Inside the Switch. ACM SIGCOMM Computer Communication Review, 44(2):44-51, April 2014.

[37] M. Bjorklund. YANG - A Data Modeling Language for The Network Configuration Protocol (NETCONF). RFC 6020, Internet Engineering Task Force (IETF), October 
2010.

[38] Z. Cai. Maestro: Achieving Scalability and Coordination in Centralized Network Control Plane. PhD Thesis, Department of Computer Science, Rice University, August 2011.

[39] M. Casado, M. Freedman, J. Pettit, J. Luo, N. McKeown, and S. Shenker. Ethane: Taking Control of the Enterprise. In Proceedings of the ACM SIGCOMM Conference on Applications, Technologies, Architectures, and Protocols for Computer Communications, volume 37, pages 1-12, Kyoto, Japan, August 2007.

[40] M. Casado, T. Garfinkel, A. Akella, M. J. Freedman, D. Boneh, N. McKeown, and S. Shenker. SANE: A Protection Architecture for Enterprise Networks. In Proceedings of the 15th USENIX Security Symposium, June 2006.

[41] O. Celebioglu, A. Saify, T. Leng, J. Hsieh, V. Mashayekhi, and R. Rooholamini. The Performance Impact of Computational Efficiency on HPC Clusters with HyperThreading Technology. In Proceedings of the 18th IEEE International Parallel and Distributed Processing Symposium, page 250, Santa Fe, New Mexico, June 2004.

[42] C. Ching-Hao and Y. Lin. OpenFlow Version Roadmap. September 2015.

[43] R. Enns, M. Bjorklund, J. Schoenwaelder, and A. Bierman. Network Configuration Protocol (NETCONF). RFC 6241, Internet Engineering Task Force (IETF), June 2011.

[44] D. Erickson. The Beacon OpenFlow Controller. In Proceedings of The Second ACM SIGCOMM Workshop on Hot Topics in Software Defined Networking (HotSDN '13), pages 13-18, Hong Kong, China, August 2013.

[45] H. Farhady, H. Lee, and A. Nakao. Software-Defined Networking: A Survey. Computer Networks: The International Journal of Computer and Telecommunications Networking, 81:79-95, April 2015. 
[46] D. Farinacci, F. Maino, V. Ermagan, Y. Hertoghs, and M. Smith. LISP Control Plane for Network Virtualization Overlays. Internet-Draft, Internet Engineering Task Force (IETF), October 2013.

[47] N. Feamster, J. Rexford, and E. Zegura. The Road to SDN: An Intellectual History of Programmable Networks. ACM SIGCOMM Computer Communication Review, 44(2):87-98, April 2014.

[48] M. Fernandez. Comparing OpenFlow Controller Paradigms Scalability: Reactive and Proactive. In Proceedings of the 27th IEEE International Conference on Advanced Information Networking and Applications (AINA), pages 1009-1016, Barcelona, Spain, June 2013.

[49] A. Greenberg, G. Hjalmtysson, D. A. Maltz, A. Myers, J. Rexford, G. Xie, H. Yan, J. Zhan, and H. Zhang. A Clean Slate 4D Approach to Network Control and Management. ACM SIGCOMM Computer Communication Review, 35(5):41-54, October 2005.

[50] N. Gude, T. Koponen, J. Pettit, B. Pfaff, M. Casado, N. McKeown, and S. Shenker. NOX: Towards an Operating System for Networks. Proceedings of ACM SIGCOMM Computer Communication Review, 38(3):105-110, July 2008.

[51] E. Haleplidis, K. Pentikousis, S. Denazis, H. Salim, D. Meyer, and O. Koufopavlou. Software-Defined Networking (SDN): Layers and Architecture Terminology. RFC 7426, Internet Engineering Task Force (IETF), January 2015.

[52] D. Harrington, B. Wijnen, and R. Presuhn. An Architecture for Describing Simple Network Management Protocol (SNMP) Management Frameworks. RFC 3411, Internet Engineering Task Force (IETF), December 2002.

[53] S. Hassas Yeganeh and Y. Ganjali. Kandoo: A Framework for Efficient and Scalable 
Offloading of Control Applications. In Proceedings of the First ACM Workshop on Hot Topics in Software Defined Networks (HotSDN '12), pages 19-24, Helsinki, Finland, August 2012.

[54] B. Heller, R. Sherwood, and N. McKeown. The Controller Placement Problem. In Proceedings of the ACM First Workshop on Hot Topics in Software Defined Networks (HotSDN '12), pages 7-12, Helsinki, Finland, August 2012.

[55] C. Hunt and B. John. Java Performance. Prentice Hall Press, October 2011.

[56] P. Ivashchenko, A. Shalimov, and R. Smeliansky. High Performance in-kernel SDN/OpenFlow Controller. In Proceedings of the USENIX Open Networking Summit, Santa Clara, California, January 2014.

[57] Y. Jarraya, T. Madi, and M. Debbabi. A Survey and a Layered Taxonomy of SoftwareDefined Networking. IEEE Communications Surveys 83 Tutorials, 16(4):1955-1980, April 2014.

[58] M. Jarschel, F. Lehrieder, Z. Magyari, and R. Pries. A Flexible OpenFlow-Controller Benchmark. In Proceedings of the 2012 European Workshop on Software Defined Networking (EWSDN), pages 48-53, Darmstadt, Germany, December 2012.

[59] R. Jones and R. Lins. Garbage Collection: Algorithms For Automatic Dynamic Memory Management. Wiley, August 1996.

[60] Z. Khattak, M. Awais, and A. Iqbal. Performance Evaluation of OpenDaylight SDN Controller. In Proceedings of the 20th IEEE International Conference on Parallel and Distributed Systems (ICPADS), pages 671-676, Hsinchu, Taiwan, December 2014.

[61] T. Koponen, M. Casado, N. Gude, J. Stribling, L. Poutievski, M. Zhu, R. Ramanathan, Y. Iwata, H. Inoue, T. Hama, and S. Shenker. Onix: A Distributed Control Platform 
for Large-scale Production Networks. In Proceedings of 9th USENIX Symposium on Operating Systems Design and Implementation (OSDI), volume 10, pages 1-6, Vancouver, British Columbia, October 2010.

[62] D. Kreutz, F. Ramos, P. Verissimo, C. Rothenberg, S. Azodolmolky, and S. Uhlig. Software-Defined Networking: A Comprehensive Survey. Proceedings of the IEEE, 103(1):14-76, January 2015.

[63] A. Lara, A. Kolasani, and B. Ramamurthy. Network Innovation using OpenFlow: A Survey. IEEE Communications Surveys $\&$ Tutorials, 16(1):493-512, August 2014.

[64] D. Marschke, J. Doyle, and P. Moyer. Software Defined Networking (SDN): Anatomy of OpenFlow Volume I. Lulu Press Inc., March 2015.

[65] N. McKeown, T. Anderson, H. Balakrishnan, G. Parulkar, L. Peterson, J. Rexford, S. Shenker, and J. Turner. OpenFlow: Enabling Innovation in Campus Networks. ACM SIGCOMM Computer Communication Review, 38(2):69-74, April 2008.

[66] J. Medved, R. Varga, A. Tkacik, and K. Gray. Opendaylight: Towards a Model-Driven SDN Controller Architecture. In Proceedings of IEEE International Symposium on a World of Wireless, Mobile and Multimedia Networks, pages 1-6, Boston, Massachusetts, June 2014 .

[67] V. Nae, R. Prodan, T. Fahringer, and A. Iosup. The Impact of Virtualization on The Performance of Massively Multiplayer Online Games. In Proceedings of the 8th IEEE Annual Workshop on Network and Systems Support for Games (NetGames), page 9, Paris, France, November 2009.

[68] J. Nakajima and V. Pallipadi. Enhancements for Hyper-Threading Technology in the Operating System: Seeking the Optimal Scheduling. In Proceedings of the 2nd USENIX 
Workshop on Industrial Experiences with Systems Software (WIESS'02), volume 2, pages 25-38, Boston, Massachusetts, December 2002.

[69] B. Nunes, M. Mendonca, X. Nguyen, K. Obraczka, and T. Turletti. A Survey of Software-Defined Networking: Past, Present, and Future of Programmable Networks. IEEE Communications Surveys \& Tutorials, 16(3):1617-1634, February 2014.

[70] S. Oaks. Java Performance: The Definitive Guide. O'Reilly Media, April 2014.

[71] S. Ortiz. Software-Defined Networking: On the Verge of a Breakthrough? IEEE Computer, 46(7):10-12, August 2013.

[72] S. Park, B. Lee, J. Shin, and S. Yang. A High-Performance IO Engine for SDN Controllers. In Proceedings of the Third IEEE European Workshop on Software Defined Networks (EWSDN), pages 121-122, London, UK, September 2014.

[73] D. Parniewicz, R. Doriguzzi Corin, L. Ogrodowczyk, M. Rashidi Fard, J. Matias, M. Gerola, V. Fuentes, U. Toseef, A. Zaalouk, B. Belter, E. Jacob, and K. Pentikousis. Design and Implementation of an OpenFlow Hardware Abstraction Layer. In Proceedings of the ACM SIGCOMM Workshop on Distributed Cloud Computing, pages 71-76, Chicago, Illinois, August 2014.

[74] M. Pearce, S. Zeadally, and R. Hunt. Virtualization: Issues, Security Threats, and Solutions. ACM Computing Surveys (CSUR), 45(2):17, February 2013.

[75] B. Pfaff and B. Davie. The Open vSwitch Database Management Protocol. RFC 7047, Internet Engineering Task Force (IETF), December 2013.

[76] K. Phemius and M. Thales. OpenFlow: Why Latency Does Matter. In Proceedings of the IFIP/IEEE International Symposium on Integrated Network Management (IM 2013), pages 680-683, Ghent, Belgium, August 2013. 
[77] Y. Rekhter, T. Li, and S. Hares. A Border Gateway Protocol 4 (BGP-4). RFC 4271, Internet Engineering Task Force (IETF), January 2006.

[78] A. Rodriguez. RESTful Web Services: The Basics. IBM developerWorks, November 2008.

[79] S. Saini, H. Jin, R. Hood, D. Barker, P. Mehrotra, and R. Biswas. The Impact of HyperThreading on Processor Resource Utilization in Production Applications. In Proceedings of the 18th IEEE International Conference on High Performance Computing (HiPC), pages 1-10, Bangalore, India, February 2011.

[80] O. Salman, I. H. Elhajj, A. Kayssi, and A. Chehab. SDN Controllers: A Comparative Study. In Proceedings of the 18th IEEE Mediterranean Electrotechnical Conference (MELECON), pages 1-6, Lemesos, Cyprus, April 2016.

[81] S. Shah, J. Faiz, M. Farooq, A. Shafi, and S. Mehdi. An Architectural Evaluation of SDN Controllers. In Proceedings of the IEEE International Conference on Communications (ICC), pages 3504-3508, Budapest, Hungary, November 2013.

[82] A. Shalimov and R. Smeliansky. On Bringing Software Engineering to Computer Networks with Software Defined Networking. Proceeding of the 7th Spring/Summer Young Researchers' Colloquium on Software Engineering (SYRCoSE 2013), May 2013.

[83] A. Shalimov, D. Zuikov, D. Zimarina, V. Pashkov, and R. Smeliansky. Advanced Study of SDN/OpenFlow Controllers. In Proceedings of the 9th ACM Central \& Eastern European Software Engineering Conference in Russia, page 1, Moscow, Russia, October 2013.

[84] J. Shetty, M. Anala, and G. Shobha. A Survey on Techniques of Secure Live Migration of Virtual Machine. Proceedings of the International Journal of Computer Applications, 39(12):34-39, February 2012. 
[85] S. Shin, Y. Song, T. Lee, S. Lee, J. Chung, P. Porras, V. Yegneswaran, J. Noh, and B. Kang. Rosemary: A Robust, Secure, and High-Performance Network Operating System. In Proceedings of the ACM SIGSAC Conference on Computer and Communications Security, pages 78-89, Scottsdale, Arizona, November 2014.

[86] M. Smith, M. Dvorkin, Y. Laribi, V. Pandey, P. Garg, and N. Weidenbacher. OpFlex Control Protocol. Internet-Draft, Internet Engineering Task Force (IETF), April 2014.

[87] A. Sonba and H. Abdalkreim. Performance Comparison of the State-of-the-art OpenFlow Controllers. MSc Thesis, Computer and Electrical Engineering Department, Halmstad University, December 2014.

[88] H. Song. Protocol-Oblivious Forwarding: Unleash the Power of SDN through a FutureProof Forwarding Plane. In Proceedings of the Second ACM SIGCOMM Workshop on Hot Topics in Software Defined Networking, pages 127-132, Hong Kong, China, August 2013.

[89] A. Tootoonchian and Y. Ganjali. HyperFlow: A Distributed Control Plane for OpenFlow. In Internet Network Management Workshop on Research on Enterprise Networking, pages 3-3, San Jose, California, April 2010.

[90] A. Tootoonchian, S. Gorbunov, Y. Ganjali, M. Casado, and R. Sherwood. On Controller Performance in Software-Defined Networks. In Proceedings of the 2nd USENIX Workshop on Hot Topics in Management of Internet, Cloud, and Enterprise Networks and Services, San Jose, California, April 2012.

[91] D. Tullsen, S. Eggers, J. Emer, H. Levy, J. Lo, and R. Stamm. Exploiting Choice: Instruction Fetch and Issue on an Implementable Simultaneous Multithreading Processor. In Proceedings of the 23rd ACM SIGARCH Annual International Symposium on Computer Architecture (ISCA '96), volume 24, pages 191-202, Philadelphia, Pennsylvania, May 1996. 
[92] D. Tullsen, S. Eggers, and H. Levy. Simultaneous Multithreading: Maximizing OnChip Parallelism. In Proceedings of the 22nd ACM SIGARCH Annual International Symposium on Computer Architecture (ISCA '95), volume 23, pages 392-403, Santa Margherita Ligure, Italy, June 1995.

[93] J. Vasseur and J. Le Roux. Path Computation Element (PCE) Communication Protocol (PCEP). RFC 5440, Internet Engineering Task Force (IETF), March 2009.

[94] G. Wang and E. Ng. The Impact of Virtualization on Network Performance of Amazon EC2 Data Center. In Proceedings of the 29th IEEE Conference on Information Communications (INFOCOM'10), pages 1-9, San Diego, California, March 2010.

[95] H. Wang, R. Shea, F. Wang, and J. Liu. On the Impact of Virtualization on Dropboxlike Cloud File Storage/Synchronization Services. In Proceedings of the 20th IEEE International Workshop on Quality of Service, page 11, Coimbra, Portugal, June 2012.

[96] G. Xu and A. Rountev. Precise Memory Leak Detection for Java Software Using Container Profiling. In Proceedings of the 30th ACM/IEEE International Conference on Software Engineering (ICSE '08), pages 151-160, Leipzig, Germany, May 2008.

[97] L. Yang, R. Dantu, T. Anderson, and R. Gopal. Forwarding and Control Element Separation (ForCES) Framework. RFC 3746, Internet Engineering Task Force (IETF), April 2004.

[98] F. Yellin and T. Lindholm. The Java Virtual Machine Specification. Addison-Wesley, January 1996.

[99] Y. Zhao, L. Iannone, and M. Riguidel. On the Performance of SDN Controllers: A Reality Check. In Proceedings of the IEEE Conference on Network Function Virtualization and Software Defined Network (NFV-SDN), pages 79-85, San Francisco, California, November 2015. 


\section{Appendix A}

\section{Controllers Settings for Performance Testing}

\section{A.1 ONOS}

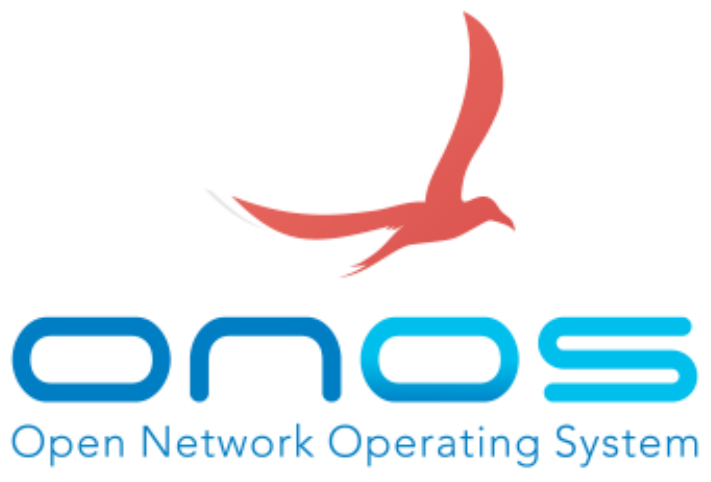

Set Java_HOME and JAVA_OPTS environment variables:

export JAVA_HOME=/usr/lib/jvm/java-8-oracle

export JAVA_OPTS="\$\{JAVA_OPTS:--Xms16G -Xmx16G\}"

Install features:

onos> app activate org.onosproject.openflow-base

onos> app activate org.onosproject.fwd

onos> cfg set org.onosproject.fwd.ReactiveForwarding packetOutOnly true

Run ONOS:

\$ cd onos-1.6.0/bin; taskset -c 0-15 ./onos-service 


\section{A.2 OpenDaylight}

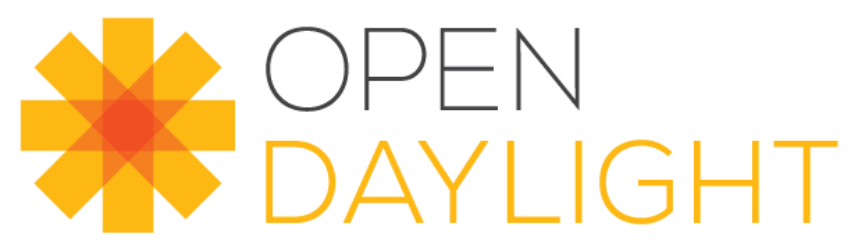

Set Java_HOME and JAVA_OPTS environment variables:

\$ export JAVA_HOME=/usr/lib/jvm/java-1.7.0-openjdk-amd64

\$ export JAVA_OPTS="\$\{JAVA_OPTS:--Xms16G -Xmx16G\}"

Install features:

opendaylight-user@root> feature:install odl-openflowplugin-flow-services-ui opendaylight-user@root> feature:install odl-openflowplugin-drop-test

Run OpenDaylight:

\$ cd distribution-karaf-0.4.2-Beryllium-SR2/bin; taskset -c 0-15 ./karaf opendaylight-user@root> dropallpacketsrpc on 


\section{A.3 Cbench Commands}

Table A.1: Cbench Command Options Description

\begin{tabular}{|c|l|}
\hline Options & \multicolumn{1}{|c|}{ Description } \\
\hline$-\mathrm{c}$ & Hostname or IP address of controller \\
\hline$-\mathrm{p}$ & Controller port \\
\hline$-\mathrm{t}$ & Test throughput instead of latency \\
\hline$-\mathrm{m}$ & Test length in ms \\
\hline$-\mathrm{l}$ & Loops per test \\
\hline$-\mathrm{s}$ & Fake $\$$ n switches \\
\hline$-\mathrm{M}$ & Unique source MAC addresses per switch \\
\hline$-\mathrm{w}$ & Loops to be disregarded on test start (warmup) \\
\hline$-\mathrm{D}$ & Delay starting testing after features_reply is received (in ms) \\
\hline$-\mathrm{i}$ & Delay between groups of switches connecting to the controller (in ms) \\
\hline$-\mathrm{I}$ & Number of switches in a connection delay group \\
\hline
\end{tabular}

Command to launch the Cbench throughput test:

$\$$ cbench -c <controller_IP_address> -p 6633 -m 5000 -1 70 -s 16 -M 100000 -w 10

-D $5000-\mathrm{t}$

Command to launch the Cbench latency test:

$\$$ cbench -c <controller_IP_address> -p 6633 -m 5000 -1 70 -s 16 -M 100000 -w 10

-i 50 -I 5 


\section{A.4 Overall Mean and Standard Deviation Calculation}

\section{Matlab Function:}

function [alln, allmean, allstd] = overallmeanstd(grpn, grpmean, grpstd)

$\%$ overall number of observations

$\operatorname{alln}=\operatorname{sum}($ grpn $) ;$

$\%$ overall Mean

allmean $=\operatorname{sum}($ grpmean $\cdot * g r p n / a l l n) ;$

\% overall Error Sum of Squares

$\mathrm{ESS}=\operatorname{sum}($ grpstd $. ` 2 . *($ grpn-1) $) ;$

$\%$ overall Group Sum of Squares

GSS $=\operatorname{sum}\left((\right.$ grpmean-allmean $) \cdot{ }^{\wedge} 2 . *$ grpn $) ;$

\% overall Standard Deviation

allstd $=\operatorname{sqrt}(($ ESS + GSS $) /($ alln-1) $) ;$

\section{Matlab Code:}

grpn $=[60 ; 60 ; 60 ; 60 ; 60]$

grpmean $=$ [meangrp1; meangrp2; meangrp3; meangrp4; meangrp5]

grpstd $=[$ stdgrp1; stdgrp2; stdgrp3; stdgrp4; stdgrp5]

[alln, allmean, allstd] = overallmeanstd(grpn, grpmean, grpstd) 


\section{A.5 Copyright Permission}

Copyright (c) 2012, Sebastian W

All rights reserved.

Redistribution and use in source and binary forms, with or without modification, are permitted provided that the following conditions are met:

* Redistributions of source code must retain the above copyright notice, this list of conditions and the following disclaimer.

* Redistributions in binary form must reproduce the above copyright notice, this list of conditions and the following disclaimer in the documentation and/or other materials provided with the distribution

THIS SOFTWARE IS PROVIDED BY THE COPYRIGHT HOLDERS AND CONTRIBUTORS "AS IS" AND ANY EXPRESS OR IMPLIED WARRANTIES, INCLUDING, BUT NOT LIMITED TO, THE IMPLIED WARRANTIES OF MERCHANTABILITY AND FITNESS FOR A PARTICULAR PURPOSE ARE DISCLAIMED. IN NO EVENT SHALL THE COPYRIGHT OWNER OR CONTRIBUTORS BE LIABLE FOR ANY DIRECT, INDIRECT, INCIDENTAL, SPECIAL, EXEMPLARY, OR CONSEQUENTIAL DAMAGES (INCLUDING, BUT NOT LIMITED TO, PROCUREMENT OF SUBSTITUTE GOODS OR SERVICES; LOSS OF USE, DATA, OR PROFITS; OR BUSINESS INTERRUPTION) HOWEVER CAUSED AND ON ANY THEORY OF LIABILITY, WHETHER IN CONTRACT, STRICT LIABILITY, OR TORT (INCLUDING NEGLIGENCE OR OTHERWISE) ARISING IN ANY WAY OUT OF THE USE OF THIS SOFTWARE, EVEN IF ADVISED OF THE POSSIBILITY OF SUCH DAMAGE.

Figure A.1: Copyright permission of MATLAB function and code 